\title{
Duality for Cousin Complexes
}

\author{
Pramathanath Sastry
}

\begin{abstract}
We relate the variance theory for Cousin complexes — $\sharp$ developed by Lipman, Nayak and the author to Grothendieck duality for Cousin complexes. Specifically for a Cousin complex $\mathcal{F}$ on $(y, \Delta)$-with $\Delta$ a codimension function on a formal scheme $y$ (noetherian, universally catenary) - and a pseudo-finite type map $f:\left(\mathcal{X}, \Delta^{\prime}\right) \rightarrow(\mathcal{Y}, \Delta)$ of such pairs of schemes with codimension functions, we show there is a derived category map $\gamma_{f}^{!}(\mathcal{F}): f^{\sharp} \mathcal{F} \rightarrow$ $f^{!} \mathcal{F}$, functorial in $\mathcal{F} \in \mathrm{Coz}_{\Delta}(y)$, inducing a functorial isomorphism $f^{\sharp} \mathcal{F} \simeq$ $E\left(f^{\sharp} \mathcal{F}\right) \stackrel{\sim}{\longrightarrow} E\left(f^{!} \mathcal{F}\right)$ (where $E$ is the Cousin functor on $\left(X, \Delta^{\prime}\right)$ ). The map $\gamma_{f}^{!}(\mathcal{F})$ is itself an isomorphism if (and clearly only if) $f^{!} \mathcal{F}$ is Cohen-Macaulay on $\left(X, \Delta^{\prime}\right)$ - which will be so, for example, whenever the complex $\mathcal{F}$ is injective or whenever the map $f$ is flat. For a fixed Cousin complex $\mathcal{F}$ on $(y, \Delta)$, $\gamma_{f}^{!}(\mathcal{F})$ is an isomorphism for every map $f$ with target $(y, \Delta)$ if and only if $\mathcal{F}$ is a complex of (appropriate) injectives. For a fixed map $f$, the functorial map $\gamma_{f}^{!}$is an isomorphism of functors if and only if $f$ is flat.

We also generalize the Residue Theorem of Grothendieck for residual complexes to Cousin complexes by defining a functorial Trace Map of graded $\mathcal{O}_{y}$-modules $\operatorname{Tr}_{f}(\mathcal{F}): f_{*} f^{\sharp} \mathcal{F} \rightarrow \mathcal{F}$ (a sum of local residues) such that when $f$ is pseudo-proper, $\operatorname{Tr}_{f}(\mathcal{F})$ is a map of complexes and the pair $\left(f^{\sharp} \mathcal{F}, \operatorname{Tr}_{f}(\mathcal{F})\right)$ represents the functor $\operatorname{Hom}\left(f_{*} \mathcal{G}, \mathcal{F}\right)$ of Cousin complexes $\mathcal{G}$ on $\left(X, \Delta^{\prime}\right)$.
\end{abstract}

\section{Contents}

1. Introduction 3

1.1. Conventions 6

2. Traces 8

2.1. Local rings 8

2.2. Trace at the graded level 9

2.3. Relative proiective space 11

2.4. The Trace Theorem 20

3. The twisted inverse image pseudofunctor 21

3.1. Factorizations 21

3.2. Flat base change 23

3.3. Comparing pseudofunctors 23

4. The comparison map 25

4.1. Pseudo-proper maps 25

4.2. Étale base change 28

5. Smooth maps 32

5.1. Verdier's isomorphism 32

The author would like to thank the Mathematisches Forschungsinstitut at Oberwolfach, Germany and the Banff International Research Station at Banff, Alberta, Canada for their help at a critical period of this research. This work was also funded by the Ganita Lab at the University of Toronto. 
5.2. Smooth pseudo-finite maps 34

5.3. The isomorphism theorem for smooth maps 36

$\begin{array}{ll}\text { 6. The Cousin of the comparison map } & 39\end{array}$

$\begin{array}{ll}\text { 6.1. Definitions and notations } & 39\end{array}$

6.2. Closed immersions 39

6.3. General maps 41

7. The Comparison map for flat morphisms 43

7.1. Tor and Ext 43

7.2. Local cohomology and the twisted inverse image 45

8. The universal property of the trace 46

8.1. Duality for Cousin complexes 46

9. Variants 50

9.1. Preliminaries 50

9.2. Twisted inverse image via residual complexes $\quad 50$

9.3. Comparison of the two twisted inverse images 51

$\begin{array}{ll}\text { References } & 56\end{array}$ 


\section{Introduction}

This paper integrates the variance theory of Cousin complexes - worked out by Lipman, Nayak and the author in [LNS - with the variance theory of the twisted inverse image ("upper shriek") occurring in Grothendieck duality for noetherian formal schemes in the form obtained by Alonso, Jeremías and Lipman in AJL2 (with a very important input from Nayak $\mathbf{N a y}$ ).

It is useful for this introduction to use the catchall symbols $-\sharp$ and $\_$! to denote in one stroke the entire variance theory for Cousin complexes in LNS and the variance theory of "upper shriek" respectively. First, let us point to two results in this paper which can be stated entirely in terms of Grothendieck duality, i.e., entirely in the framework of - !. To fix ideas we will (for this paragraph) restrict ourselves to ordinary noetherian excellent schemes admitting codimension functions $^{1}$. The first one, viz. Theorem 7.2 .2 says that if $f: X \rightarrow Y$ is a separated finite type map and $\Delta$ is a codimension function on $Y$, then the twisted inverse image functor $f^{!}$takes Cohen-Macaulay complexes (with respect to $\Delta$ ) to CohenMacaulay complexes (with respect to $f^{\sharp} \Delta$, where $f^{\sharp} \Delta$ is as in [NS Example 2.1.2]) if and only if $f$ is flat. The second result (viz. Theorem 6.3.5) concerns Gorenstein complexes on $(Y, \Delta)$, i.e., complexes $\mathcal{F}$ which are Cohen-Macaulay with respect to $\Delta$ and such that the associated Cousin complex $E_{\Delta} \mathcal{F}$ is a complex of injective quasicoherent $\mathcal{O}_{Y}$-modules (cf. Hrt p. 248] where Hartshorne unnecessarily restricts himself to bounded complexes). We show that $\mathcal{F}$ is Gorenstein with respect to $\Delta$ if and only if $f^{!} \mathcal{F}$ is Cohen-Macaulay with respect to $f^{\sharp} \Delta$ for every separated finite type map $f$ whose target is $Y$.

The above results are not our main results, but they are the ones that could be stated without referring to the constructions in [LNS and therefore provide a kinder gentler introduction for the lay reader to our work. However, the proofs of the just stated results (which are valid for formal schemes) require an understanding of the interrelationships between $-\sharp$ and - ! (the main theme of this paper).

The constructions of $\_\sharp$ and $\_$! are in spirit and outlook antithetical. The approach to Grothendieck duality in AJL2 and Nay is global, top down and holistic - in the spirit of Deligne and Verdier (cf. D1, D2 and $\mathbf{V}]$ ). One begins by providing _ ' for pseudo-proper maps by global methods; and then works one's way downward (via the flat base change theorem AJL2, Theorem 7.4]) to pseudofinite type maps which are composites of compactifiable maps by showing that _ is local. This is not straightforward - the stumbling block being the fact that in the category of formal schemes a closed subscheme of an open subscheme of a scheme $X$ need not be an open subscheme of a closed subscheme of $X$. The "localness" of _! is proved by Nayak Nay via a surprising twist of Deligne's argument for ordinary schemes.

At the other extreme is the construction of the variance theory $\_$* The outlook from the outset is punctual (complete local rings!). And reductionist in the following sense; the initial search is for the basic irreducible units (the atoms) of such a theory at the level of formal neighborhoods of points and sheaves supported there-i.e. at the level of complete local rings and zero-dimensional modules (cf. Hu1]). In such an approach it has to be an article of faith that these local (punctual) constructions

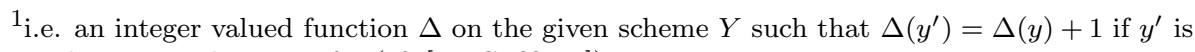
an immediate specialization of $y$ (cf. [LNS $\S \S 2.1]$ ).
} 
somehow link up and give a global canonical variance theory. This faith is not misplaced and in LNS the core result is that given data of the form

$$
D=(X \stackrel{f}{\rightarrow} \mathcal{Y}, \Delta, \mathcal{F})
$$

where $f$ is a morphism in the category $\mathbb{F}$ of [LNS] (which is included in the category of formal schemes with codimension functions on them, and essentially pseudo-finite type maps), $\Delta$ is a codimension function on $y$ and $\mathcal{F}$ is a Cousin complex on $(y, \Delta)$, there is a Cousin complex $f^{\sharp} \mathcal{F}$ on $\left(X, f^{\sharp} \Delta\right)$ which is functorial in complexes $\mathcal{F}$ with a fixed codimension function $\Delta$. Here $f^{\sharp} \Delta$ is the codimension function on $X$ induced by $f$ as in [LNS 2.1.2]. The basic units for this construction are modules on complete local rings $\left(R, \mathfrak{m}_{R}\right)$ such that $\Gamma_{\mathfrak{m}_{R}} M=M$ (i.e. $M$ is a zero-dimensional $R$-module). In Hu1, I-Chiau Huang worked out a variance theory - \# for such objects with respect to local homomorphisms $\left(R, \mathfrak{m}_{R}\right) \stackrel{\varphi}{\rightarrow}\left(S, \mathfrak{m}_{S}\right)$ of complete local rings such that the residue field extension $k_{R} \rightarrow k_{S}$ is finitely generated. For $D$ as above and a point $x \in \mathcal{X}$ with $f(x)=y$, one has a map $\varphi^{x}: \widehat{\mathcal{O}}_{y, y} \rightarrow \widehat{\mathcal{O}}_{x, x}$ and the functor $f^{\sharp}$ is so constructed that - among other properties that it enjoys - it satisfies the relation $\left(f^{\sharp} \mathcal{F}\right)(x)=\varphi_{\#}^{x} \mathcal{F}(f(x))$. The construction is done in such a way that $-\sharp$ is a variance theory, i.e. it is a pseudofunctor.

What then do these theories have to do with each other? ${ }^{2}$ For data $D$ with $f$ a composite of compactifiable maps (so that $f^{!}$exists) there seems no obvious way to compare $f^{\sharp} \mathcal{F}$ and $f^{!} \mathcal{F}$. And the hope (admittedly faint) that the two might be abstractly isomorphic in $\mathbf{D}(\mathcal{X})$ is dashed by the counter-example obtained by setting (with $k$ a field) $X=\operatorname{Spec}(k), y=\operatorname{Spec}(k[T]), f$ the map given by the natural map $(T \mapsto 0): k[T] \rightarrow k$, and $\mathcal{F}=f_{*} k^{\sim}$. The natural codimension function $\Delta$ on $y$ for the Cousin complex $\mathcal{F}$ is the one which gives the closed point of $y$ value zero. In this instance $f^{\sharp} \mathcal{F}=k^{\sim}$ and $f^{!} \mathcal{F}=k^{\sim} \oplus k^{\sim}[1]$. In fact $f^{!} \mathcal{F}$ is not even Cohen-Macaulay (with respect to the codimension function $\Delta^{\prime}=0$ ) and so is far from being isomorphic to $f^{\sharp} \mathcal{F}$. However, note that $E\left(f^{!} \mathcal{F}\right)$ is indeed isomorphic to $f^{\sharp} \mathcal{F}$, where $E$ is the Cousin functor associated with $\Delta^{\prime}\left(=f^{\sharp} \Delta\right)$.

We show that this is true in general for data $D$. We also investigate when $f^{\sharp} \mathcal{F}$ and $f^{!} \mathcal{F}$ are isomorphic. The bridge between the two theories is a derived category comparison map $f^{\sharp} \mathcal{F} \rightarrow f^{!} \mathcal{F}$ which on applying the (appropriate) Cousin functor $E$ transforms to an isomorphism. The key to obtaining this map is the observation that if the point $x$ is closed in its fiber, then with $M=\mathcal{F}(y), R=\widehat{\mathcal{O}}_{y, y}$ and $S=\widehat{\mathcal{O}}_{x, x}$ there is an $R$-linear punctual trace map $\operatorname{Tr}_{f, x}(\mathcal{F}): \varphi_{\#}^{x} M \rightarrow M$ which induces an isomorphism $\Phi: \varphi_{\#}^{x} M \stackrel{\sim}{\longrightarrow} \operatorname{Hom}_{R}^{c}(S, M)$ Hu1 Chapter 7]. This allows one to have a map of graded $\mathcal{O}_{y}$-modules

$$
\operatorname{Tr}_{f}(\mathcal{F}): f_{*} f^{\sharp} \mathcal{F} \rightarrow \mathcal{F} .
$$

Our principal results are the following.

1) (The Trace Theorem) If $f$ in $D$ is pseudo-proper then the trace $\operatorname{map} \operatorname{Tr}_{f}(\mathcal{F})$ is a map of complexes (cf. Theorem 2.4.2(a)). The crucial step here is the proof of the trace theorem for (relative) projective space over ordinary schemes. This takes up a considerable amount of time, and involves digressions into residues and the residue theorem for projective space. Once the trace theorem is established for projective space, then the proof given in $\mathbf{H r t}$ for residual complexes applies without change

${ }^{2} \mathrm{I}$ am aware that this might be come under the heading of "setting up a straw man" but this was indeed my original confusion. 
to our situation. The punctual trace $\operatorname{Tr}_{f, x}$ has a transitivity property which now gives the following result (cf. Theorem 2.4.2 (b)): let $g: \mathcal{W} \rightarrow X$ be a second pseudoproper map. Then identifying $(f g)^{\sharp} \mathcal{F}$ with $g^{\sharp} f^{\sharp} \mathcal{F}$ (a part of the variance theory for $-\sharp$ ) we have

$$
\operatorname{Tr}_{f g} \mathcal{F}=\operatorname{Tr}_{f}(\mathcal{F}) \circ f_{*} \operatorname{Tr}_{g}\left(f^{\sharp} \mathcal{F}\right) .
$$

2) First suppose $f$ as above is pseudo-proper. Let $\left(f^{!}, \tau_{f}\right)$ be the dualizing pair given by Grothendieck duality. By the universal property of $\left(f^{!}, \tau_{f}\right)$, the map $\operatorname{Tr}_{f}(\mathcal{F})$ gives rise to a map

$$
\gamma_{f}^{\prime}(\mathcal{F}): f^{\sharp} \mathcal{F} \rightarrow f^{!} \mathcal{F}
$$

such that $\tau_{f}(\mathcal{F}) \circ \mathbf{R} f_{*}\left(\gamma_{f}^{!}\right)=\operatorname{Tr}_{f}(\mathcal{F})$ (cf. 4.1.1)). We are implicitly identifying $\mathbf{R} f_{*} \mathcal{F}$ with $f_{*} \mathcal{F}$ since $\mathcal{F}$, being a Cousin complex, is flasque. Next suppose $f$ compactifiable. Then $\gamma_{f}^{!}$can be defined as $v^{*} \gamma_{\bar{f}}^{!}$where $v$ is an open immersion, $\bar{f}$ a pseudo-proper map and $f=\bar{f} v$. We show that this is independent of the compactification $(v, \bar{f})$ of $f$. We actually show more. Suppose $f$ is a composite of compactifiable maps. Then applying the just described process repeatedly one can define $\gamma_{f}^{!}$. We show in Theorem 4.1.4 that this is independent of the factorization of $f$ as a composite of compactifiables, and that this comparison map respects pseudofunctoriality (in a sense made precise by Theorem 4.1.4(b)). We point out that in the "classical" situation (i.e., ordinary schemes ...), a separated finite type map is always compactifiable by a theorem of Nagata $[\mathbf{N}]$. (See also $[\mathbf{L u}$ and $[\mathbf{D 3}$.)

3) Suppose $f$ in $D$ is a composite of compactifiable maps. Set $f^{E} \mathcal{F}=E\left(f^{!} \mathcal{F}\right)$ where $E=E_{f^{\sharp} \Delta}$ is the Cousin associated with the codimension function $f^{\sharp} \Delta$ on $X$. Applying $E$ to $\gamma_{f}^{!}$we obtain a map of Cousin complexes on $\left(X, f^{\sharp} \Delta\right)$,

$$
\gamma_{f}^{E}(\mathcal{F}): f^{\sharp} \mathcal{F} \rightarrow f^{E} \mathcal{F} .
$$

Theorem 6.3.1 states that $\gamma_{f}^{E}(\mathcal{F})$ is an isomorphism. The proof is not straightforward. The crucial step is Theorem 5.3.2 which states that if $f$ is smooth then the map $\gamma_{f}^{!}(\mathcal{F})$ is an isomorphism (note that the last is a statement about $\gamma_{f}^{!}$which is stronger than the corresponding statement about $\gamma_{f}^{E}$ ). The proof here is involved and in the end amounts understanding Grothendieck duality for smooth maps as well as the compatibility of local and global duality in terms of endomorphisms of residual complexes. This result on smooth maps takes up all of Section 5. Now a general $f$ of the type we are considering can be locally factored as a closed immersion followed by a smooth map, whence we are reduced to showing $\gamma_{f}^{E}(\mathcal{F})$ is an isomorphism when $f$ is a closed immersion. This is easily proven (cf. Corollary 6.2.5.11.

4) A consequence of the circle of ideas above is Theorem 6.3.2 which states that $\gamma_{f}^{!}(\mathcal{F})$ is an isomorphism if (and clearly only if) $f^{!} \mathcal{F}$ is Cohen-Macaulay with respect to $f^{\sharp} \Delta$. We use ideas from Suominen $[\mathbf{S u}]$ who shows that (with a fixed codimension function) the category of Cohen-Macaulay complexes is equivalent to the category of Cousin complexes. Now $f^{!} \mathcal{F}$ being Cohen-Macaulay can be viewed as a condition on $\mathcal{F}$ (if $f$ is allowed to vary) or as a condition on $f$ (if $\mathcal{F}$ is allowed to vary within its codimension class). For fixed $\mathcal{F}$ we show that $\gamma_{f}^{!}(\mathcal{F})$ is an isomorphism for every composite of compactifiable maps $f$ with target $(\mathcal{y}, \Delta)$ if and only if $\mathcal{F}$ is a complex of $\mathcal{A}_{\text {qct }}(y)$-injectives. Here $\mathcal{A}_{\text {qct }}(y)$ is the category of quasi-coherent $\mathcal{O}_{y}$ modules $\mathcal{F}$ which are torsion, i.e., satisfying $\Gamma_{y}^{\prime} \mathcal{F}=\mathcal{F}$ where $\Gamma_{y}^{\prime}$ is as in the last paragraph of AJL2 1.2.1]. In particular $\gamma_{f}^{\prime}(\mathcal{F})$ is an isomorphism when $\mathcal{F}$ is residual. At 
the other end, for a fixed map $f:\left(X, \Delta^{\prime}\right) \rightarrow(y, \Delta)$, we show that $\gamma_{f}^{!}(\mathcal{F})$ is an isomorphism of complexes - for all Cousin complexes $\mathcal{F}$ with respect to $\Delta$-if and only if $f$ is flat. (Cf. Theorem 6.3.3 and Theorem 7.2.2) We have already stated these results (at the beginning of this introduction) entirely in the framework of - !

5) We show (cf. Theorem 8.1.10) that given data of the form $\left(\left(X, \Delta^{\prime}\right) \stackrel{f}{\rightarrow}\right.$ $(\mathcal{y}, \Delta), \mathcal{F})$ where $f$ is a pseudo-proper map and $\mathcal{F}$ is a Cousin complex on $(\mathcal{Y}, \Delta)$, the pair $\left(f^{\sharp} \mathcal{F}, \operatorname{Tr}_{f}(\mathcal{F})\right)$ represents the functor $\operatorname{Hom}_{y}\left(f_{*} \mathcal{G}, \mathcal{F}\right)$ of Cousin complexes on $\left(\mathcal{X}, \Delta^{\prime}\right)$, i.e., the pair $\left(f^{\sharp} \mathcal{F}, \operatorname{Tr}_{f}(\mathcal{F})\right)$ induces an isomorphism

$$
\operatorname{Hom}_{\Delta^{\prime}}\left(\mathcal{G}, f^{\sharp} \mathcal{F}\right) \stackrel{\sim}{\longrightarrow} \operatorname{Hom}_{y}\left(f_{*} \mathcal{G}, \mathcal{F}\right) .
$$

(Here the left side is the group of morphisms in $\mathrm{Coz}_{\Delta^{\prime}}$ between $\mathcal{G}$ and $f^{\sharp} \mathcal{F}$.) In other words the variance theory $\mathbb{Z}^{\sharp}$, together with the trace maps (TR) for pseudo-proper maps $f$, is a true duality theory for Cousin complexes.

One consequence is this. Suppose $f:\left(X, \Delta^{\prime}\right) \rightarrow(\mathcal{Y}, \Delta)$ is a finite map of schemes and for $\mathcal{F} \in \mathrm{Coz}_{\Delta}(y), f^{b} \mathcal{F} \in \mathrm{Coz}_{\Delta^{\prime}}(X)$ is the unique complex of quasicoherent $\mathcal{O}_{x}$-modules satisfying $f_{*} f^{b} \mathcal{F}=\mathcal{H} o m_{\mathfrak{y}}\left(f_{*} \mathcal{O}_{x}, \mathcal{F}\right)$, then a certain obvious isomorphism of the graded $\mathcal{O}_{x}$ modules $f^{\sharp} \mathcal{F}$ and $f^{b} \mathcal{F}$ is an isomorphism of complexes (cf. Corollary 8.1.11).

6) In certain circumstances we can define the twisted inverse image of $f$ even if $f$ is not a composite of open immersions and pseudo-proper maps. In Section 9 we imitate the theory given in $\mathbf{H r t}$ to obtain this when $y$ in the data $D$ has a bounded residual complex on it. For this we apply the variance theory - $\#$ of LNS to residual complexes. In this case we define $f^{!} \mathcal{E}$ via residual complexes for objects $\mathcal{E}$ in $\mathbf{D}(y)$ such that $\mathbf{R} \Gamma_{y}^{\prime} \mathcal{E}$ is in $\mathbf{D}^{+}(y)$ and such that there exists a complex $\mathcal{G}$ with coherent homology sheaves satisfying $\mathbf{R} \Gamma_{y}^{\prime} \mathcal{E} \simeq \mathbf{R} \Gamma_{y}^{\prime} \mathcal{G}$. We also show that this construction is indeed $f^{!} \mathcal{E}$ when $f$ is a composite of compactifiable maps (cf. Theorem 9.3.10).

1.1. Conventions. In addition to the notations and conventions in $\mathbf{L N S}$ 1.4] we use the following in this paper. Minor differences in notation between this paper and [LNS] are also noted (see item 1 below).

(1) We use the upright symbol $\mathrm{H}^{i}$ for cohomology rather than the slanted symbol $H^{i}$ used in LNS. Further if $\mathcal{X}$ is a scheme and $\mathcal{J}$ an $\mathcal{O}_{X}$ ideal then we use $\Gamma_{\mathcal{J}}$ for the functor $\underset{\underset{n}{\lim }}{\operatorname{Hom}} \operatorname{Hom}_{\mathcal{O}}\left(\mathcal{O}_{x} / \mathcal{J}^{n},-\right)$ and the symbol $\Gamma_{\mathcal{J}}$ for the sheafified version $\lim \mathcal{H}_{\stackrel{n}{n}}\left(\mathcal{O}_{x} / \mathcal{J}^{n},-\right)$. In [LNS the slanted version $\Gamma$ is the one used throughout (the context determining the interpretation to be placed).

(2) For a locally noetherian formal scheme $X$,

$$
\widetilde{\mathbf{D}}_{\mathrm{qc}}(X):=\mathbf{R} \Gamma_{x}^{\prime-1}\left(\mathbf{D}_{\mathrm{qc}}(X)\right)
$$

is the triangulated subcategory of $\mathbf{D}(\mathcal{X})$ whose objects are those complexes $\mathcal{F}$ such that $\mathbf{R} \Gamma_{X}^{\prime} \mathcal{F} \in \mathbf{D}_{\mathrm{qc}}(\mathcal{X})$-or equivalently, $\mathbf{R} \Gamma_{X}^{\prime} \mathcal{F} \in \mathbf{D}_{\mathrm{qct}}(\mathcal{X})$.

(3) For $X$ as above, $\mathbf{D}_{\mathrm{c}}^{*}(X)$ is the essential image of $\mathbf{D}_{\mathrm{c}}(\mathcal{X})$ under $\mathbf{R} \Gamma_{X}^{\prime}$, i.e., $\mathbf{D}_{\mathrm{c}}^{*}(X)$ is the full subcategory of $\mathbf{D}(X)$ such that $\mathcal{E} \in \mathbf{D}_{\mathrm{c}}^{*}(X) \Leftrightarrow \mathcal{E} \simeq \mathbf{R} \Gamma_{X}^{\prime} \mathcal{F}$ with $\mathcal{F} \in \mathbf{D}_{\mathrm{c}}(\mathcal{X})$.

(4) For $\mathcal{X}$ as above,

$$
\widetilde{\mathbf{D}}_{\mathrm{c}}^{*}(X):=\mathbf{R} \Gamma_{X}^{\prime-1}\left(\mathbf{D}_{\mathrm{c}}^{*}(X) \cap \mathbf{D}^{+}(X)\right)
$$


is the triangulated subcategory of $\mathbf{D}(X)$ whose objects are complexes $\mathcal{F}$ such that $\mathbf{R} \Gamma_{X}^{\prime} \mathcal{F} \in \mathbf{D}_{\mathrm{c}}^{*}(X) \cap \mathbf{D}^{+}(X)$.

(5) If $f: X \rightarrow y$ is a morphism in $\mathbb{F}$ which is pseudo-proper, then we denote the resulting trace map by $\tau_{f}: \mathbf{R} f_{*} f^{!} \rightarrow \mathbf{1}_{\mathbf{D}(y)}$. (See AJL2 Theorem 6.1 (a)]).

(6) The category $\mathbb{G}$ has as objects noetherian formal schemes, and its morphisms are maps of noetherian formal schemes which are composites of compactifiable maps, or equivalently, composites of open immersions and pseudo-proper maps.

(7) The category $\mathbb{F}^{*}$ has the same objects as $\mathbb{F}$ but its morphisms are composites of compactifiable maps, or equivalently, composites of open immersions and pseudo-proper maps, i.e., $\mathbb{F}^{*}=\mathbb{F} \cap \mathbb{G}$. Note that if $f: X \rightarrow y$ is a map in $\mathbb{G}$ and $y \in \mathbb{F}$ then $X \in \mathbb{F}$ (cf. [LNS 2.1.2]) whence $f$ is a map in $\mathbb{F}^{*}$. The category $\mathbb{F}_{c}^{*}$ has the same objects as $\mathbb{F}_{c}$, and its morphisms are maps in $\mathbb{F}_{c}$ such that the underlying map of formal schemes is in $\mathbb{F}^{*}$.

(8) The category $\mathbb{F}^{r}$ is the full subcategory of $\mathbb{F}$ consisting of objects admitting a bounded residual complex LNS $\S \S 9.1]$. Note that if $\mathcal{x} \stackrel{f}{\rightarrow}$ y is a map in $\mathbb{F}$ and $y$ is an object in $\mathbb{F}^{r}$ then $X$ is an object in $\mathbb{F}^{r}$ (cf. LNS Prop. 9.1.4]). The category $\mathbb{F}_{c}^{r}$ is the full subcategory of $\mathbb{F}_{c}$ consisting of objects $(X, \Delta)$ such that $X \in \mathbb{F}^{r}$. Note that if $\left(X, \Delta^{\prime}\right) \stackrel{f}{\rightarrow}(\mathcal{Y}, \Delta)$ is a map in $\mathbb{F}_{c}$ and $(y, \Delta) \in \mathbb{F}_{c}^{r}$, then $\left(X, \Delta^{\prime}\right) \in \mathbb{F}_{c}^{r}$.

(9) For $(X, \Delta) \in \mathbb{F}_{c}, \operatorname{CM}(X, \Delta)$ is the full subcategory of $\mathbf{D}_{\text {qct }}^{+}(X)$ consisting of complexes which are Cohen-Macaulay with respect to $\Delta$, i.e. if $\mathbf{D}_{\mathrm{CM}}^{+}(X ; \Delta)$ is as in LNS $\left.\S \S 3.3\right]$ then $\operatorname{CM}(X, \Delta):=\mathbf{D}_{\mathrm{CM}}^{+}(X ; \Delta) \cap \mathbf{D}_{\mathrm{qct}}(X)$. The category $\operatorname{CM}^{*}(X, \Delta)$ is the full subcategory of $\operatorname{CM}(X, \Delta)$ given by $\mathrm{CM}^{*}(X, \Delta)=\operatorname{CM}(X, \Delta) \cap \mathbf{D}_{\mathrm{c}}^{*}(X)$.

(10) If $\boldsymbol{-}^{\natural}$ is a contravariant pseudofunctor [LNS $\left.\S 4\right]$ on a category $\mathfrak{C}$ then, for a pair of maps $X \stackrel{f}{\rightarrow} Y \stackrel{g}{\rightarrow} Z$ in $\mathfrak{C}$, we write $C_{f, g}^{\natural}$ for the resulting isomorphism of functors $f^{\natural} g^{\natural} \stackrel{\sim}{\longrightarrow}(g f)^{\natural}$. A similar convention applies for covariant pseudofunctors.

(11) The important "pseudofunctor" from the point of view of Grothendieck duality is the twisted inverse image "pseudofunctor" (see Section 3). Unfortunately when we deal with non-ordinary schemes, this is not a pseudofunctor. Indeed, if $X$ is a noetherian scheme, then $\mathbf{1}_{X}^{!}=\mathbf{R} \Gamma_{x}^{\prime}$ which is not in general isomorphic to $\mathbf{1}_{\widetilde{\mathbf{D}}_{\mathrm{qc}}^{+}(x)}$. The twisted inverse image is a prepseudofunctor in the sense of Lipman. A (contravariant) pre-pseudofunctor $-^{\natural}$ is data of the form

$$
\left((-)^{\natural},(-)^{\natural}, C_{(-),(-)}^{\natural}, \delta_{(-)}^{\natural}\right)
$$

satisfying the requirements of [LNS $\S 4]$ for a pseudofunctor, with the exception that $\delta_{X}$ is no longer required to be isomorphism of functors for $X \in \mathfrak{C}$. We will often abuse terminology and refer to pre-pseudofunctors as pseudofunctors.

(12) Let $f: X \rightarrow y$ be a closed immersion of formal schemes (see GD p. 442]). Let $\mathcal{J}$ be the kernel of the surjective map $\mathcal{O}_{y} \rightarrow f_{*} \mathcal{O}_{x}$, and let $\bar{y}$ be the 
ringed space $\left(y, \mathcal{O}_{y} / \mathcal{J}\right)$. The natural map $X \rightarrow \bar{y}$ is denoted $\bar{f}$. Note that $\bar{f}$ is flat and $f$ factors naturally as $X \stackrel{\bar{f}}{\rightarrow} \bar{y} \stackrel{i}{\rightarrow} y$.

(13) A formally étale map of formal schemes is a formally smooth map which is of relative dimension 0 . Such a map is called étale if it is essentially of pseudo-finite type. Equivalently an étale map is a smooth map of relative dimension 0. Relative dimension here is as in [LNS Definition 2.6.2].

(14) A map $\left(X, \Delta^{\prime}\right) \stackrel{f}{\rightarrow}(\mathcal{Y}, \Delta)$ in $\mathbb{F}_{c}$ (or in $\mathbb{F}_{c}^{*}, \mathbb{F}_{c}^{r}$ ) is smooth, étale, pseudoproper etc., if the underlying map $X \rightarrow y$ of formal schemes is smooth, étale, pseudo-proper etc.

(15) For $(X, \Delta) \in \mathbb{F}_{c}$ and $\mathcal{F}, \mathcal{G} \in \mathrm{Coz}_{\Delta}(X)$

$$
\operatorname{Hom}_{\Delta}(\mathcal{F}, \mathcal{G}):=\operatorname{Hom}_{\mathrm{Coz}_{\Delta}}(\mathcal{F}, \mathcal{G}) .
$$

(16) For $X \in \mathbb{F}, Q_{X}$ will denote all the localization functors from subcategories of $\mathbf{K}(X)$ to $\mathbf{D}(X)$. The source of $Q_{X}$ will be clear from the context. If the subcategory is $\operatorname{Coz}_{\Delta}(X)$ for a codimension function $\Delta$ on $X$, then we may sometimes restrict the target of $Q_{X}$ to $\operatorname{CM}(X, \Delta)$ (so that $Q_{X}$ becomes an equivalence between $\operatorname{Coz}_{\Delta}(X)$ and $\left.\operatorname{CM}(X, \Delta)\right)$. This will also be clear from the context.

(17) If $R$ is a local ring, $\mathfrak{m}_{R}$ will denote its maximal ideal and $k_{R}$ the residue field $R / \mathfrak{m}_{R}$. A 0 -dimensional $R$-module $M$ is (as in Hu1) a module satisfying $\Gamma_{\mathfrak{m}_{R}} M=M$.

(18) Let $x$ be a point on a noetherian (formal) scheme $X$, and let $M$ be a 0 dimensional $\mathcal{O}_{x, x}$ module. Then $i_{x} M$ will denote the sky-scraper sheaf on $x$ whose sections are $M$ over open sets containing $x$ and zero otherwise. Note that $i_{x} M$ is a quasi-coherent $\mathcal{O}_{x}$-module (cf. [LNS Lemma 2.3.5]).

(19) As in [LNS], for a complete local ring $A, A_{\sharp}$ will denote the category of 0 -dimensional $A$-modules. The pseudofunctor on complete local rings given in [LNS, Theorem 4.3.1] will be denoted $-_{\sharp}$.

\section{Traces}

In this section we define a trace map

$$
\operatorname{Tr}_{f}(\mathcal{F}): f_{*} f^{\sharp} \mathcal{F} \longrightarrow \mathcal{F}
$$

associated with the data $(f, \mathcal{F})$ where $f:\left(X, \Delta^{\prime}\right) \rightarrow(\mathcal{Y}, \Delta)$ is a morphism in $\mathbb{F}_{c}$ and $\mathcal{F}$ is a Cousin complex on $(\mathcal{Y}, \Delta)$. More precisely $\operatorname{Tr}_{f}(\mathcal{F})$ is a map from Fgt $f_{*} f^{\sharp} \mathcal{F} \rightarrow$ Fgt $\mathcal{F}$ where Fgt is the forgetful functor from complexes to graded objects. The most important result in this section is the Trace Theorem, i.e., Theorem 2.4.2 which asserts that $\operatorname{Tr}_{f}(\mathcal{F})$ is compatible with coboundary maps on $f_{*} f^{\sharp} \mathcal{F}$ and $\mathcal{F}$ (i.e., $\operatorname{Tr}_{f}(\mathcal{F})$ is a map of complexes) when $f$ is pseudo-proper. Related results may be found in $\mathbf{H u 2}$.

Before we treat traces, we begin with more preliminary material involving local rings associated with a point on a formal scheme.

2.1. Local rings. If $X$ is an ordinary scheme and $x$ a point on $X$, then there are two naturally occurring local rings associated with the point $x$ - the stalk $\mathcal{O}_{x}$ of the structure sheaf $\mathcal{O}_{X}$ at $x$ and the completion $\widehat{\mathcal{O}}_{x}$ of $\mathcal{O}_{x}$ at its maximal ideal. For a formal (non-ordinary) scheme there are four local rings that one can legitimately attach to a point as we shall see. 
To simplify the discussion let $X=\operatorname{Spf}(A, I)$, where $(A, I)$ is a noetherian adic ring. For $f \in A$, as is standard in such situations, we set $A_{\{f\}}$ equal to the $I$-adic completion of $A_{f}$. The points of $X$ are in one-to-one correspondence with open prime ideals of $A$, and the closed points of $X$ correspond to maximal ideals of $A$ (which are necessarily open in the $I$-adic topology, since $A$ is complete in the $I$-adic topology, whence $I$ is contained in the Jacobson radical of $A$ ). Let $x$ be a point on $X$, and let $\mathfrak{p} \subset A$ be the open prime ideal corresponding to $x$. We then have an obvious local ring associated with $x$, viz. $A_{\mathfrak{p}}$. However, this ring is not the stalk of $\mathcal{O}_{x}$ at $x$. One verifies that the following formula gives the stalk at $x$ :

$$
A_{\{\mathfrak{p}\}}:=\lim _{\tilde{f \notin p}} A_{\{f\}}=\mathcal{O}_{X, x} .
$$

We have a canonical map

$$
A_{\mathfrak{p}} \longrightarrow A_{\{\mathfrak{p}\}}
$$

whose $I$-adic completion is an isomorphism (see proof of AJL2 Lemma 7.1.1]). Denote the common $I$-adic completion of $A_{\mathfrak{p}}$ and $A_{\{\mathfrak{p}\}}$ by $A_{[\mathfrak{p}]}$ and the common $\mathfrak{p}-$ adic completion of $A_{\mathfrak{p}}, A_{\{\mathfrak{p}\}}$ and $A_{[\mathfrak{p}]}$ by $\widehat{A}_{\mathfrak{p}}$. We then have faithfully flat inclusions of local rings

$$
A_{\mathfrak{p}} \subset A_{\{\mathfrak{p}\}} \subset A_{[\mathfrak{p}]} \subset \widehat{A}_{\mathfrak{p}} .
$$

We also have formal schemes

$$
X_{[\mathfrak{p}]}:=\operatorname{Spf}\left(A_{[\mathfrak{p}]}, I A_{[\mathfrak{p}]}\right)
$$

and

$$
x_{\mathfrak{p}}^{*}:=\operatorname{Spf}\left(\widehat{A}_{\mathfrak{p}}, \mathfrak{p} \widehat{A}_{\mathfrak{p}}\right)
$$

together with natural étale maps (see 1.1130)

$$
x_{\mathfrak{p}}^{*} \longrightarrow x_{[\mathfrak{p}]} \longrightarrow x
$$

Note that the map $X_{[\mathfrak{p}]} \rightarrow X$ is adic, but the map $X_{\mathfrak{p}}^{*} \rightarrow X_{[\mathfrak{p}]}$ (as well as the above composite) need not be so.

2.2. Trace at the graded level. Let $-_{\sharp}$ be as in 1.119). Suppose $\varphi: R \rightarrow S$ is a morphism in $\mathfrak{C}_{\mathbf{r f}}$-i.e., $R, S$ are complete noetherian local rings and $\varphi$ is a local homomorphism with $k_{S}$ a finite $k_{R}$-algebra - and suppose $M$ is an object in $R_{\sharp}$. According to Hu1, Chapter 7 ], we have an $R$-linear trace map, functorial in $M \in R_{\sharp}$

$$
\operatorname{Tr}_{S / R, M}: \varphi_{\sharp} M \longrightarrow M
$$

such that the induced map

$$
\Phi_{S / R, M}: \varphi_{\sharp} M \longrightarrow \operatorname{Hom}_{R}^{c}(S, M)
$$

is an isomorphism of $S$-modules. Moreover if $\varphi$ is surjective, 2.2.2 recovers the isomorphism in [NS Theorem 4.3.1I(ii)]. Note that we are using the fact that $R_{\sharp}=R_{\#}, S_{\sharp}=S_{\#}$ and $\varphi_{\sharp}=\varphi_{\#}$ where -\# is Huang's pseudofunctor on $\mathfrak{C}$, the category of of complete noetherian local rings and local homomorphisms (cf. proof

of [LNS Theorem 4.3.1], especially Lemma 4.3.2). Further, by the commutative 
diagram on the top of [Hu1 p. 51], if $\xi: S \rightarrow T$ is a second morphism in $\mathfrak{C}_{\mathbf{r f}}$ then the diagram

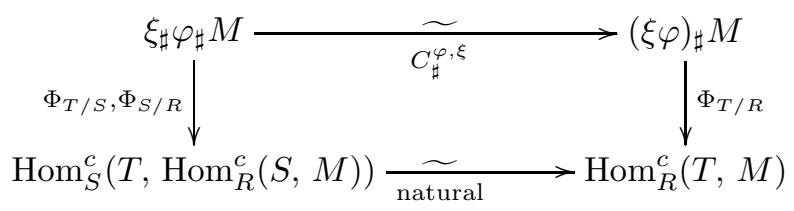

commutes. We are using the fact that the transcendence degrees of the residue field extension $k_{R} \rightarrow k_{S}$ and $k_{S} \rightarrow k_{T}$ are zero, whence $C_{\sharp}^{\varphi, \xi}=C_{\#}^{\varphi, \xi}$ by the construction of $-_{\sharp}$ from $-_{\#}$ via [LNS Lemma 4.3.2] in the proof of [Ibid, Theorem 4.3.1]. As a consequence the following diagram commutes

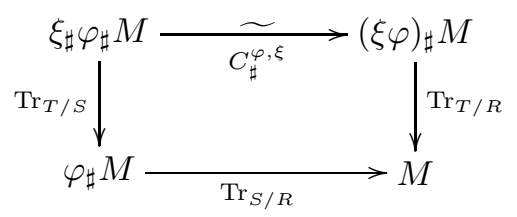

Next consider datum of the form

$$
D=\left(\left(X, \Delta^{\prime}\right) \stackrel{f}{\rightarrow}(y, \Delta), \mathcal{F}, x\right)
$$

where $f$ is a map in $\mathbb{F}_{c}, \mathcal{F}$ is an object in $\operatorname{Coz}_{\Delta}(y)$ and $x$ is a point on $\mathcal{X}$. Associated to $D$ we define a punctual trace at $x$, obviously functorial in $\mathcal{F}$,

$$
\operatorname{Tr}_{f, x}(\mathcal{F})=\left\{\begin{array}{l}
\operatorname{Tr}_{S / R, M}: \varphi_{\sharp} M \rightarrow M \\
0 \quad \text { otherwise }
\end{array} \quad \text { if } \quad \Delta^{\prime}(x)=\Delta(f(x))\right.
$$

where, in the first case, $R$ and $S$ are the completions of the local rings at $x$ and $y=f(x)$ respectively, $\varphi: R \rightarrow S$ is the natural map induced by $f$, and $M$ is $\mathcal{F}(y)$. We remind the reader that the condition $\Delta^{\prime}(x)=\Delta(f(x))$ is equivalent to $x$ being closed in the fiber of $f$ over $f(x))$. Varying $x$, we get a map of graded $\mathcal{O}_{y}$-modules

$$
\operatorname{Tr}_{f}(\mathcal{F}): f_{*} f^{\sharp} \mathcal{F} \rightarrow \mathcal{F}
$$

given by

$$
\operatorname{Tr}_{f}(\mathcal{F})=\sum_{x \in \mathcal{X}} i_{f(x)} \operatorname{Tr}_{f, x}(\mathcal{F}) .
$$

More precisely, as we noted before, the source of $\operatorname{Tr}_{f}(\mathcal{F})$ is $f_{*} \operatorname{Fgt}_{x} f^{\sharp} \mathcal{F}$ and the target is $\mathrm{Fgt}_{y} \mathcal{F}$ where $\mathrm{Fgt}$ is the forgetful functor from complexes to graded modules.

If $g:\left(\mathcal{W}, \Delta^{\prime \prime}\right) \rightarrow\left(\mathcal{X}, \Delta^{\prime}\right)$ is a second map in $\mathbb{F}_{c}$ then from (2.2.3) we see that the diagram

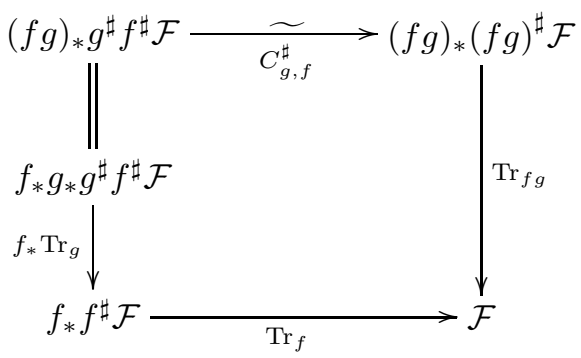

commutes. 
We end this subsection by discussing $\operatorname{Tr}_{f}$ when $f: X \rightarrow Y$ is a closed immersion. By LNS $\S \S 8.2$, Def. 8.3.1 and $\S \S 8.4]$, for $\mathcal{F} \in \mathrm{Coz}_{\Delta}(y)$ we have an isomorphism

$$
f_{*} f^{\sharp} \mathcal{F} \stackrel{\sim}{\longrightarrow} \mathcal{H o m}:\left(f_{*} \mathcal{O}_{x}, \mathcal{F}\right) .
$$

We then have

Lemma 2.2.8. Let $f:\left(\mathcal{X}, \Delta^{\prime}\right) \rightarrow(\mathcal{Y}, \Delta)$ be a closed immersion in $\mathbb{F}_{c}$. Let $\boldsymbol{e}: \mathcal{H o m}_{\mathfrak{y}}\left(f_{*} \mathcal{O}_{x}, \mathcal{F}\right) \rightarrow \mathcal{F}$ be "evaluation at 1 ". Then $\operatorname{Tr} f(\mathcal{F})$ is the composite

$$
f_{*} f^{\sharp} \mathcal{F} \stackrel{2.2 .7}{\longrightarrow} \mathcal{H o m} \cdot \dot{y}\left(f_{*} \mathcal{O}_{x}, \mathcal{F}\right) \stackrel{e}{\rightarrow} \mathcal{F} .
$$

In particular $\operatorname{Tr}_{f}(\mathcal{F})$ is a map of complexes.

Proof. This follows from [NS $\S \S 8.2(75)]$ and from the fact that the map in [LNS Thm. 4.3.1 I(ii)] is the map (2.2.2) when $\varphi$ is a surjective map.

2.3. Relative projective space. The key step for proving the general Trace Theorem is the Trace Theorem for relative projective spaces $\pi: \mathbb{P}_{Y}^{d} \rightarrow Y$, after which the proof in Hrt VII $\S 2$,pp. 369-373] applies mutatis mutandis.

Throughout this subsection $Y$ is a fixed ordinary noetherian scheme. Let

$$
\pi=\pi_{Y}: \mathbb{P}:=\mathbb{P}_{Y}^{d} \longrightarrow Y
$$

be the relative projective space of fiber dimension $d$ over $Y$, i.e., $\pi$ is the first projection in the decomposition $\mathbb{P}^{d}=Y \times_{\mathbb{Z}} \mathbb{P}_{\mathbb{Z}}^{d}$. There is a well known isomorphism

$$
\int_{\pi}: \mathrm{R}^{d} \pi_{*} \omega_{\pi} \stackrel{\sim}{\longrightarrow} \mathcal{O}_{Y}
$$

(cf. [EGA-III 2.1.12] or Hrt p.152, Theorem 3.4]); the generating section $\sigma$ of $\mathrm{R}^{d} \pi_{*} \omega_{\pi}$ corresponding to the standard section 1 of $\mathcal{O}_{Y}$ described as follows. We have $\mathbb{P}=\operatorname{Proj}\left(\mathcal{O}_{Y}\left[T_{0}, \ldots, T_{d}\right]\right)$. Let $\mathcal{U}=\left(U_{i}\right)_{i=0}^{d}$ be the open cover of $\mathbb{P}$ given by $U_{i}=\left\{T_{i} \neq 0\right\}$. On $U_{0} \cap \ldots \cap U_{d}$ we have inhomogeneous coordinates $t_{i}=T_{i} / T_{0}$, $i=1, \ldots, d$ whence a section

$$
\check{\sigma}_{T}:=\frac{\mathrm{d} t_{1} \wedge \ldots \wedge \mathrm{d} t_{d}}{t_{1} \ldots t_{d}} \in \omega_{\pi}\left(U_{0}, \ldots, U_{d}\right) .
$$

We have an isomorphism

$$
\mathrm{R}^{d} \pi_{*} \omega_{\pi} \stackrel{\sim}{\longrightarrow} \mathrm{H}^{d}\left(\pi_{*} \check{\mathcal{C}} \bullet\left(\mathcal{U}, \omega_{\pi}\right)\right)
$$

and $\check{\sigma}_{T}$ has a natural image in the right side as a Čech cohomology class. Let $\sigma$ be the corresponding element on the left side. The section $\sigma$ does not depend on the choice of homogeneous coordinates $T_{0}, \ldots, T_{d}$ of $\mathbb{P}$ (cf. [C. p.34, Lemma 2.3.1]) and is the sought after section.

It is well known (and easily verified from the description above) that $\int_{\pi}$ is compatible with arbitrary base changes $Y^{\prime} \rightarrow Y$. If $Y$ is affine, say $Y=\operatorname{Spec}(A)$, then define

$$
\int_{\mathbb{P} / Y}: \mathrm{H}^{d}\left(\mathbb{P}, \omega_{\pi}\right) \longrightarrow A
$$

as the global section of $\int_{\pi}$.

Since the global trace map $\operatorname{Tr}_{\pi}$ is built out of punctual traces, we look into these traces in somewhat greater detail now. Suppose $\varphi: R \rightarrow S$ is a smooth 
residually finite map of relative dimension $d$ between complete local rings and $M$ is an object in $R_{\sharp}$. From [LNS Theorem 4.3.1, I(i)] we have

$$
\varphi_{\sharp} M \stackrel{\sim}{\sim} \mathrm{H}_{\mathfrak{m}_{S}}^{d}\left(M \otimes \omega_{S / R}\right)
$$

and this isomorphism is functorial in $M$. This gives us a residue map

$$
\operatorname{res}_{S / R, M}: \mathrm{H}_{\mathfrak{m}_{S}}^{d}\left(M \otimes \omega_{S / R}\right) \rightarrow M
$$

defined by the commutativity of

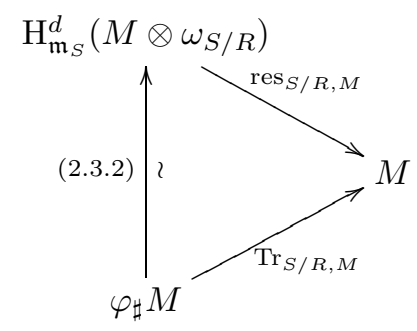

where $\operatorname{Tr}_{S / R, M}$ is as in (2.2.1). If $S$ is of the form

$$
S=R\left[t_{1}, \ldots, t_{d}\right]_{\mathfrak{p}}
$$

where $t_{1}, \ldots, t_{d}$ are independent variables over $R$, then Huang has explicit formulae for $\operatorname{res}_{S / R}$ (cf. [Hu1, p.42,(7.1)] and [Hu1, bottom of p.21]) based on an iterated form of the Tate residue.

Recall again that in this subsection $Y$ is a fixed noetherian ordinary scheme.

Proposition 2.3.4. Suppose $Y$ is an affine noetherian scheme, $x$ a closed point on $\mathbb{P}$ closed in the fiber of $\pi$ over $y=\pi(x)$ and $M$ a zero-dimensional $\mathcal{O}_{Y, y^{-}}$ module. Denote $\widehat{\mathcal{O}}_{Y, y}, \widehat{\mathcal{O}}_{X, x}$ and $i_{y} M$ by $R, S$ and $\mathcal{F}$ respectively. Let $W$ be the closure of $x$ in $\mathbb{P}$. Then the diagram below-with unlabelled arrows the natural maps-commutes.

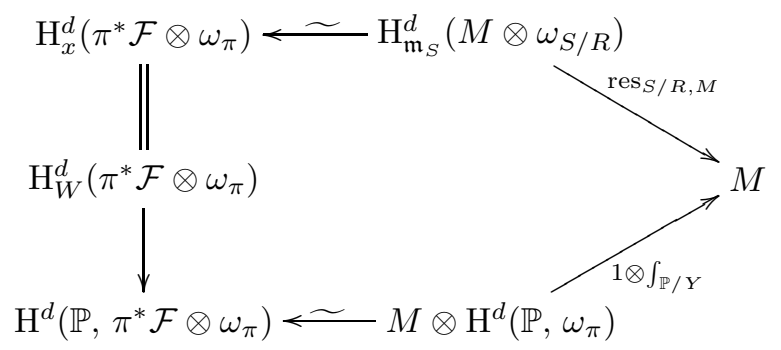

Proof. First we elaborate on the equality on the top of the left column in the above diagram. Let $Y^{\prime}=\operatorname{Spec} R, y^{\prime} \in Y^{\prime}$ the closed point of $Y^{\prime}, Y^{\prime} \stackrel{g}{\rightarrow} Y$, $\mathbb{P}^{\prime}:=\mathbb{P}_{Y^{\prime}}^{d} \stackrel{g^{\prime}}{\rightarrow} \mathbb{P}$ the natural affine maps, $\pi^{\prime}: \mathbb{P}^{\prime} \rightarrow Y^{\prime}$ the natural projection and $F$ the common fiber $\pi^{\prime-1}\left(y^{\prime}\right)=\pi^{-1}(y)$. Since the fiber $F$ is shared by $\mathbb{P}$ and $\mathbb{P}^{\prime}$ therefore we have a point $x^{\prime} \in \mathbb{P}^{\prime}$ corresponding to $x \in \mathbb{P}$. Let $\mathcal{G}=\pi^{*} \mathcal{F} \otimes \omega_{\pi}$ and $\mathcal{G}^{\prime}=g^{\prime *} \mathcal{G}\left(=\pi^{\prime *}\left(g^{*} \mathcal{F}\right) \otimes \omega_{\pi^{\prime}}\right)$. It is not hard to see that

$$
\mathrm{H}_{x^{\prime}}^{d}\left(\mathcal{G}^{\prime}\right)=\mathrm{H}_{x}^{d}(\mathcal{G}) .
$$


More generally we remind the reader that if $(B, \eta)$ is a local ring, $N$ a $B$-module, and $(\widehat{B}, \widehat{\eta})$ the completion of $(B, \eta)$ at $\eta$, then for every integer $i$ we have $\mathrm{H}_{\eta}^{i}(N)=$ $\mathrm{H}_{\eta}^{i}(N) \otimes_{B} \widehat{B}=\mathrm{H}_{\widehat{\eta}}^{i}\left(N \otimes_{B} \widehat{B}\right) .^{3}$

Now, $\Gamma_{F} \mathcal{G}^{\prime}=\mathcal{G}^{\prime}$ and $\underline{\mathrm{H}}_{F}^{i}\left(\mathcal{G}^{\prime}\right):=\mathrm{H}^{i}\left(\Gamma_{F} \mathcal{G}^{\prime}\right) \simeq \pi^{\prime *} i_{y^{\prime}}\left(\mathrm{H}_{\mathfrak{m}_{y^{\prime}}}^{i}(M)\right) \otimes \omega_{\pi^{\prime}}=0$ for $i>0$, since $M$ is a zero dimensional $R$-module. One can therefore find an $\mathcal{O}_{Y^{\prime}}$-injective resolution $\mathcal{G}^{\prime} \rightarrow \mathcal{J}$ such that $\Gamma_{F} \mathcal{J}=\mathcal{J}$, i.e. an injective resolution (topologically) supported on $F$ (by replacing any injective resolution $\mathcal{J}$ by $\mathcal{J}=\Gamma_{F} \mathcal{J}$ ). Moreover, $g^{\prime}$ being affine, $g_{*}^{\prime} \mathcal{J}$ is a resolution of $g_{*}^{\prime} \mathcal{G}^{\prime}=g_{*}^{\prime} g^{\prime *}\left(\pi^{*} \mathcal{F} \otimes \omega_{\pi}\right)=g_{*}^{\prime} g^{\prime *}\left(\pi^{*} \mathcal{F}\right) \otimes \omega_{\pi}=\mathcal{G}$ (the last equality follows from the fact that $M$ is a zero dimensional $R$-module, whence $\mathcal{F}=g_{*} g^{*} \mathcal{F}$ giving $\left.g_{*}^{\prime} g^{\prime *}\left(\pi^{*} \mathcal{F}\right)=\pi^{*} \mathcal{F}\right)$. Let $z \in \mathbb{P}$ be such that $\pi(z)=y$, and let $z^{\prime} \in \mathbb{P}^{\prime}$ be the point lying over $y^{\prime}$ corresponding to $z$. Since the local rings $\mathcal{O}_{\mathbb{P}, z}$ and $\mathcal{O}_{\mathbb{P}^{\prime}, z}$ have the same completion for such pairs of points - i.e. the natural map $\mathcal{O}_{\mathbb{P}, z} \rightarrow \mathcal{O}_{\mathbb{P}^{\prime}, z^{\prime}}$ transforms into an equality on completing - and since $\Gamma_{F} \mathcal{J}=\mathcal{J}$, therefore it is not hard to see that $g_{*}^{\prime} \mathcal{J}$ consists of $\mathcal{O}_{Y}$-injectives. Thus $g_{*}^{\prime} \mathcal{J}$ is an injective resolution of $\mathcal{G}$. We therefore have

$$
\begin{aligned}
\mathrm{H}_{W}^{d}(\mathcal{G})=\mathrm{H}^{d}\left(\Gamma_{W} g_{*}^{\prime} \mathcal{J}\right) & =\mathrm{H}^{d}\left(\Gamma_{g^{\prime-1} W} \mathcal{J}\right) \\
& =\mathrm{H}^{d}\left(\Gamma_{x^{\prime}} \mathcal{J}\right) \\
& =\mathrm{H}_{x^{\prime}}^{d}\left(\mathcal{G}^{\prime}\right) \\
& =\mathrm{H}_{x}^{d}(\mathcal{G})
\end{aligned}
$$

which explains the top of the left column.

Let $\widetilde{\mathrm{res}}_{S / R, M}: \mathrm{H}_{\mathfrak{m}_{S}}^{d}\left(M \otimes \omega_{S / R}\right) \rightarrow M$ be the composite

$$
\begin{aligned}
\mathrm{H}_{\mathfrak{m}_{S}}^{d}\left(M \otimes \omega_{S / R}\right) & \stackrel{\sim}{\longrightarrow} \mathrm{H}_{x}^{d}\left(\pi^{*} \mathcal{F} \otimes \omega_{\pi}\right) \\
& =\mathrm{H}_{W}^{d}\left(\pi^{*} \mathcal{F} \otimes \omega_{\pi}\right) \\
& \longrightarrow \mathrm{H}^{d}\left(\mathbb{P}, \pi^{*} \mathcal{F} \otimes \omega_{\pi}\right) \\
& \sim M \otimes \mathrm{H}^{d}\left(\mathbb{P}, \omega_{\pi}\right) \\
& \longrightarrow M
\end{aligned}
$$

where the last arrow is $1 \otimes \int_{\mathbb{P} / Y}$. We have to show that $\operatorname{res}_{S / R}=\widetilde{\operatorname{res}}_{S / R}$.

Since $\int_{\pi}$ is compatible with the flat base change $\operatorname{Spec}(R) \rightarrow Y$, and so are all other functorially defined arrows in the diagram, we assume without loss of generality that $Y=\operatorname{Spec}(R)$. Assume - again without loss of generality - that $x$ lies in the open subscheme $U_{0}=\operatorname{Spec}\left(R\left[T_{1} / T_{0}, \ldots, T_{d} / T_{0}\right]\right)$ of $\mathbb{P}=\operatorname{Proj}\left(R\left[T_{0}, \ldots, T_{d}\right]\right)$. We therefore have

$$
S=R\left[T_{1} / T_{0}, \ldots, T_{d} / T_{0}\right]_{\mathfrak{p}}
$$

for some maximal ideal $\mathfrak{p}$ of the polynomial ring $R\left[T_{1} / T_{0}, \ldots, T_{d} / T_{0}\right]$ and $\mathbf{H u 1}$, p.42,(7.1)] applies. If the natural extension $k_{R} \rightarrow k_{S}$ is trivial then $S=R\left[\left[t_{1}, \ldots, t_{d}\right]\right]$ for some analytically independent variables $t_{1}, \ldots, t_{d}$ over $R$ and the formula in loc.cit. reduces to

$$
\operatorname{res}_{S / R}\left[\begin{array}{c}
m \otimes \mathrm{d} t_{1} \wedge \cdots \wedge \mathrm{d} t_{d} \\
t_{1}^{\alpha_{1}}, \ldots, t_{d}^{\alpha_{d}}
\end{array}\right]= \begin{cases}m & \text { if } \alpha_{1}=\alpha_{2}=\cdots=\alpha_{d}=1 \\
0 & \text { otherwise }\end{cases}
$$

\footnotetext{
${ }^{3}$ In LNS $\left.\S \S 4.2,(18)\right]$ we made an elaborate distinction between these modules, necessary, to make sure that our book-keeping was correct when constructing the pseudofunctor $-^{\sharp}$.
} 
Suppose we are in this situation, i.e., $k_{R}=k_{S}$ or equivalently $S=R\left[\left[t_{1}, \ldots, t_{d}\right]\right]$. Since the two maps, $\int_{\pi}$ and $\operatorname{res}_{S / R}$, are compatible with change of homogeneous coordinates $T_{0}, \ldots, T_{d}$ (cf. Hu1 p.49,(7.7)] for the latter assertion), we may assume without loss of generality that $t_{i}=T_{i} / T_{0}$ for $i=1, \ldots, d$. Let $\mathbf{t}=\left(t_{1}, \ldots, t_{d}\right)$, and let $Z \hookrightarrow U_{0}$ be the closed subscheme given by the vanishing of the $t_{i}$ 's. We define

$$
\widetilde{\text { res }_{\mathbf{t}}}: \mathrm{H}_{\mathbf{t} S}^{d}\left(\omega_{S / R}\right) \longrightarrow R
$$

by the commutativity of

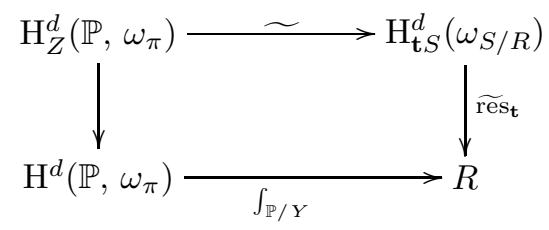

Lipman's proof Lp1 p. 75, Prop. (8.6)] of his "Residue Theorem for Projective Space" applies without change to our situation ${ }^{4}$ and we conclude that

$$
\widetilde{\operatorname{res}}_{\mathbf{t}}\left[\begin{array}{c}
p(\mathbf{t}) \mathrm{d} t_{1} \wedge \cdots \wedge \mathrm{d} t_{d} \\
t_{1}^{\alpha_{1}}, \ldots, t_{d}^{\alpha_{d}}
\end{array}\right]=p_{\alpha_{1}-1, \ldots, \alpha_{d}-1}
$$

where the right-side is the coefficient of $t_{1}^{\alpha_{1}-1} \ldots t_{d}^{\alpha_{d}-1}$ in the power series $p(\mathbf{t})$. It is straightforward to check that the diagram

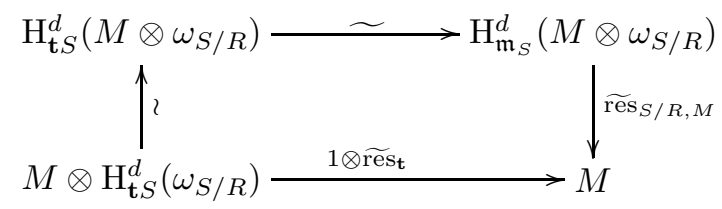

commutes. Whence, on comparing (2.3.4.1) with (2.3.4.2), we see that $\widetilde{\mathrm{res}}_{S / R}=$ $\operatorname{res}_{S / R}$ in this case, i.e. when the natural extension $k_{R} \rightarrow k_{S}$ is trivial.

One can reduce to the above case by making a flat base change $R \rightarrow R^{\prime}$ with $R^{\prime}$ a complete noetherian local ring satisfying $\mathfrak{m}_{R} R^{\prime}=\mathfrak{m}_{R}$. In greater detail, suppose $k_{S}=k_{R}\left[\theta_{1}, \ldots, \theta_{m}\right]$. We can find $R^{\prime}$ as above such that for each $i=1, \ldots, m$, the minimal polynomial of $\theta_{i}$ over $k_{R}$ splits into a product of linear factors over $k_{R^{\prime}}$. Let $\mathbb{P}^{\prime}=R^{\prime} \otimes_{R} \mathbb{P}$ and let the resulting projections be $p: \mathbb{P}^{\prime} \rightarrow \mathbb{P}$ and $\pi^{\prime}: \mathbb{P}^{\prime} \rightarrow \operatorname{Spec}\left(R^{\prime}\right)$. Let $\left\{x_{1}, \ldots, x_{n}\right\}=p^{-1}(x)$ and for $i=1, \ldots, m$ let $S_{i}^{\prime}$ be the completion of $\mathcal{O}_{\mathbb{P}^{\prime}, x_{i}}$. If $M^{\prime}=R \otimes_{R} M$, then according to Hu1 p.47, Lemma (7.5)] we have

$$
\sum_{i=1}^{n} \operatorname{res}_{S_{i}^{\prime} / R^{\prime}, M^{\prime}} \circ \tau=\tau_{o} \circ \operatorname{res}_{S / R, M}
$$

where $\tau$ is the natural map

$$
\mathrm{H}_{\mathfrak{m}_{S}}^{d}\left(M \otimes \omega_{S / R}\right) \longrightarrow R^{\prime} \otimes \mathrm{H}_{\mathfrak{m}_{S}}^{d}\left(M \otimes \omega_{S / R}\right)=\bigoplus_{i=1}^{n} \mathrm{H}_{\mathfrak{m}_{S_{i}}}^{d}\left(M^{\prime} \otimes \omega_{S_{i}^{\prime} / R^{\prime}}\right)
$$

and $\tau_{o}$ is the natural map

$$
M \rightarrow R^{\prime} \otimes_{R} M=M^{\prime} .
$$

\footnotetext{
${ }^{4}$ In fact since $(1: 0: \cdots: 0)$ are the "homogeneous coordinates" of the $R$-valued point $\operatorname{Spec}(R) \stackrel{\sim}{\longrightarrow} Z \hookrightarrow \mathbb{P}$, only that part of the proof of loc.cit. which concerns itself with rational points applies, i.e. the part that begins at the bottom of p.77.
} 
On the other hand, since $\int_{\mathbb{P} / Y}$ is compatible with base change, clearly we have

$$
\sum_{i=1}^{n} \widetilde{\operatorname{res}}_{S_{i}^{\prime} / R^{\prime}, M^{\prime}} \circ \tau=\tau_{o} \circ \widetilde{\operatorname{res}}_{S / R, M}
$$

Since $k_{S_{i}^{\prime}}=k_{R^{\prime}}$, we have $\widetilde{\operatorname{res}}_{S_{i}^{\prime}} / R^{\prime}=\operatorname{res}_{S_{i}^{\prime} / R^{\prime}}$. Now $\tau_{o}$ is an injective map and hence (2.3.4.3) and (2.3.4.4) give the Proposition.

Proposition 2.3.5. (Trace Theorem for Projective Space.) Let $Y$ be in $\mathbb{F}$ and $\mathcal{F}$ in $\mathrm{Coz}_{\Delta}(Y)$ for some codimension function $\Delta$ on $Y$. Then the map of graded $\mathcal{O}_{Y}$-modules

$$
\operatorname{Tr}_{\pi}(\mathcal{F}): \pi_{*} \pi^{\sharp} \mathcal{F} \longrightarrow \mathcal{F}
$$

is a map of complexes

Proof. Our strategy is as follows. We will show that there is a complex $\mathcal{E}=\mathcal{E}_{\mathcal{F}}$ of quasi-coherent $\mathcal{O}_{Y}$-modules associated with $\pi^{\sharp} \mathcal{F}$ together with maps of complexes (the second one an isomorphism)

$$
\alpha: \pi_{*} \pi^{\sharp} \mathcal{F} \rightarrow \mathcal{E}
$$

and

$$
\beta: \mathcal{E} \stackrel{\sim}{\longrightarrow} \mathcal{F} \otimes \mathrm{R}^{d} \pi_{*} \omega_{\pi}
$$

such that the diagram of graded $\mathcal{O}_{Y}$-modules

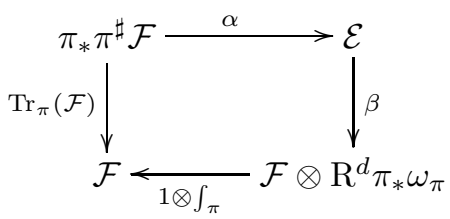

commutes. Since all arrows other than $\operatorname{Tr}_{\pi}(\mathcal{F})$ are maps of complexes, this would prove that $\operatorname{Tr}_{\pi}(\mathcal{F})$ is a map of complexes.

The complex $\mathcal{E}$ is best described as the $E_{1}$ term of a spectral sequence associated to a natural filtration of the complex $\pi_{*} \pi^{\sharp} \mathcal{F}$. Recall from [LNS $\left.\S \S 10.2\right]$ (especially the discussion following the proof of 10.2.4 in Ibid.) that we have a decreasing filtration $\left\{F^{p}\right\}_{p \in \mathbb{Z}}$ of subcomplexes of $\pi^{\sharp} \mathcal{F}$ given by

$$
F^{p}=\pi^{\sharp}\left(\sigma_{\geq p} \mathcal{F}\right),
$$

and recall that $F^{p} / F^{p+1}=\pi^{\sharp}\left(\mathcal{F}^{p}[-p]\right)$ LNS (89)]. $\quad F^{\bullet}$ induces a decreasing filtration $\left\{\Phi^{p}\right\}_{p \in \mathbb{Z}}$ on $\pi_{*} \pi^{\sharp} \mathcal{F}$ obtained by applying $\pi_{*}$ to $F^{\bullet}$. Let $\left\{E_{r}^{p, q}\right\}$ be the associated spectral sequence. Then $\mathcal{E}$ is the complex

$$
\cdots \rightarrow E_{1}^{p-1,0} \stackrel{\partial_{\mathcal{E}}^{p-1}}{\longrightarrow} E_{1}^{p, 0} \stackrel{\partial_{\mathcal{E}}^{p}}{\longrightarrow} E_{1}^{p+1,0} \rightarrow \ldots
$$

where $\partial_{\mathcal{E}}$ is the natural coboundary on the $E_{1}$ term of the spectral sequence $\left\{E_{r}^{p, q}\right\}$.

To say more (i.e. to define $\alpha$ and $\beta$ ) we need to deconstruct the above definition of $\mathcal{E}$. We view $\pi^{\sharp} \mathcal{F}$ (resp. $\pi_{*} \pi^{\sharp} \mathcal{F}$ ) as a bigraded complex (not necessarily a double complex!) whose $p$-th column is the complex $F^{p} / F^{p+1}\left(\right.$ resp. $\left.\Phi^{p} / \Phi^{p+1}\right)$. In greater detail let

$$
\mathcal{A}^{p, q}:=\left(F^{p} / F^{p+1}\right)^{p+q} .
$$


Then, with $\Delta^{\prime}=\pi^{\sharp} \Delta$, we have

$$
\mathcal{A}^{p, q}=\bigoplus_{\substack{\Delta(\pi(x))=p \\ \Delta^{\prime}(x)=p+q}} i_{x}\left(\pi^{\sharp} \mathcal{F}\right)(x) .
$$

In LNS $\S \S 10.1]$ the above sheaf is denoted $\mathcal{E}^{p+q, p}$. One has the following decomposition of $\mathcal{O}_{\mathbb{P}}-$ modules

$$
\left(\pi^{\sharp} \mathcal{F}\right)^{n}=\bigoplus_{p+q=n} \mathcal{A}^{p, q} \quad F^{p}=\bigoplus_{r \geq p} \mathcal{A}^{r, q}
$$

as well as the associated decomposition of $\mathcal{O}_{Y}$-modules

$$
\left(\pi_{*} \pi^{\sharp} \mathcal{F}\right)^{n}=\bigoplus_{p+q=n} \pi_{*} \mathcal{A}^{p, q} \quad \Phi^{p}=\bigoplus_{r \geq p} \pi_{*} \mathcal{A}^{r, q} .
$$

Note that the map

$$
\partial^{p, q, k}: \mathcal{A}^{p, q} \rightarrow \mathcal{A}^{p+k, q-k+1}
$$

induced by the coboundary map on $\mathcal{F}^{\sharp}$ is such that $\partial^{p, q, k}=0$ if $k$ is negative, i.e. there are no arrows with a westward component in the bigraded complex (cf. LNS Lemma 10.2.3]).

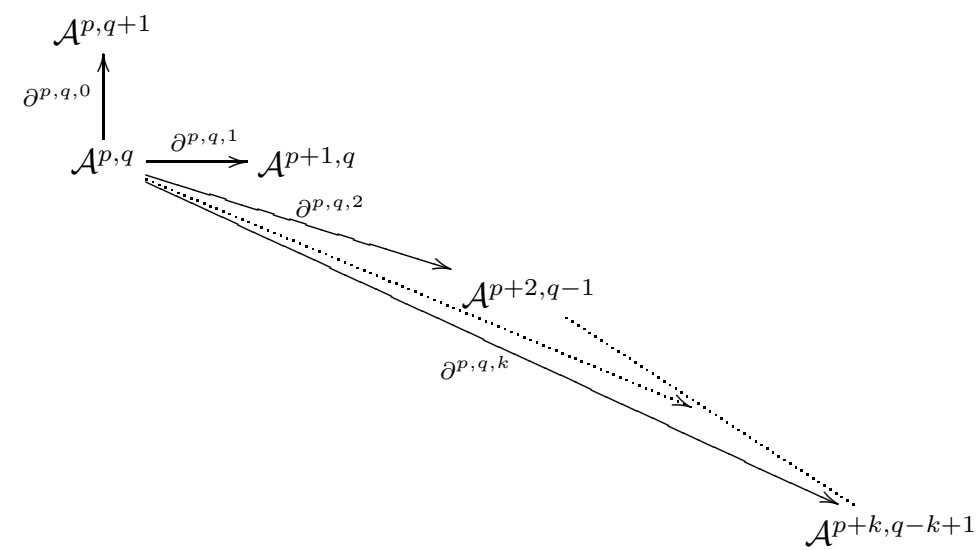

Recall that the $E_{1}$ term of $\left\{E_{r}^{p, q}\right\}$ has a simple description which in our case translates to having

$$
\mathcal{E}^{p}=\mathrm{H}^{p}\left(\Phi^{p} / \Phi^{p+1}\right)
$$

such that the coboundary map $\partial_{\mathcal{E}}^{p}$ is the connecting homomorphism associated to the short exact sequence of complexes

$$
0 \rightarrow \Phi^{p+1} / \Phi^{p+2} \rightarrow \Phi^{p} / \Phi^{p+2} \rightarrow \Phi^{p} / \Phi^{p+1} \rightarrow 0 .
$$

Note that $\Phi^{p} / \Phi^{p+1}=\pi_{*}\left(F^{p} / F^{p+1}\right)$ is the complex whose $(p+q)$-th homogeneous piece is $\pi_{*} \mathcal{A}^{p, q}$ and whose coboundary on $(p+q)$-cochains is $\pi_{*} \partial^{p, q, 0}$. The complex $\Phi^{p} / \Phi^{p+2}$ is a two column double complex ${ }^{5}$; its left column - the $p$-th column-being

$$
\Phi^{p} / \Phi^{p+1}=\pi_{*} \pi^{\sharp}\left(\mathcal{F}^{p}[-p]\right)
$$

and its right column - the $(p+1)$-th column —-being

$$
\Phi^{p+1} / \Phi^{p+2}=\pi_{*} \pi^{\sharp}\left(\mathcal{F}^{p+1}[-p-1]\right) .
$$

\footnotetext{
${ }^{5}$ Which means, for this proof, the grids anti-commute.
} 


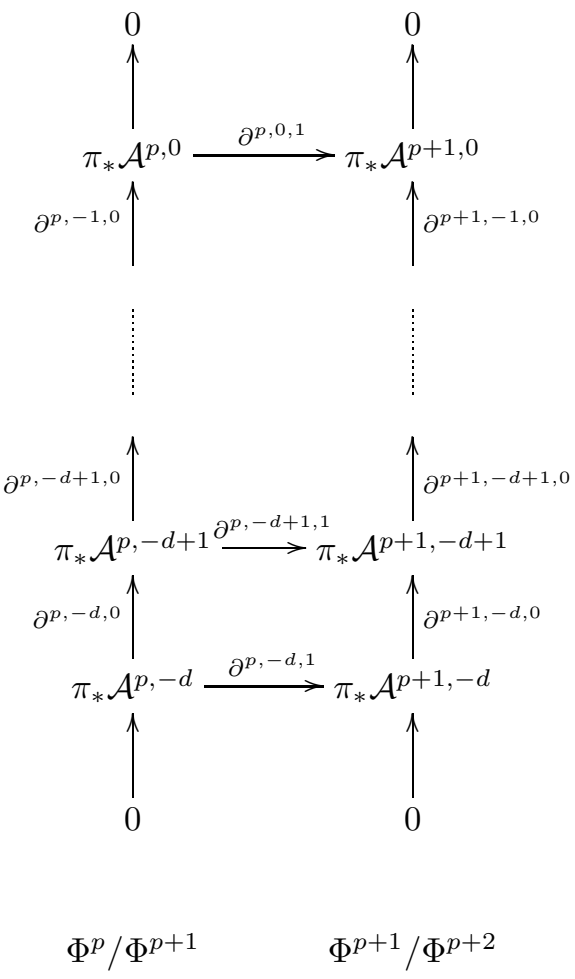

Now $\mathcal{A}^{p, q}=0$ for $q>0$ and hence $\Phi^{p} / \Phi^{p+1}$ has no terms in degrees $k>p$. Therefore we have a natural surjective map

$$
\bar{\alpha}^{p}=\psi^{p}(\mathcal{F}): \pi_{*} \mathcal{A}^{p, 0}=\left(\Phi^{p} / \Phi^{p+1}\right)^{p} \rightarrow \mathrm{H}^{p}\left(\Phi^{p} / \Phi^{p+1}\right)=\mathcal{E}^{p} .
$$

For future reference we note that

$$
\bar{\alpha}^{p}(\mathcal{F})=\psi^{p}\left(\mathcal{F}^{p}[-p]\right) .
$$

The map $\alpha: \pi_{*} \pi^{\sharp} \mathcal{F} \rightarrow \mathcal{E}$ at the graded level is defined in degree $p$ by the composite

$$
\left(\pi_{*} \pi^{\sharp} \mathcal{F}\right)^{p} \stackrel{\text { projection }}{\longrightarrow} \pi_{*} \mathcal{A}^{p, 0} \stackrel{\psi^{p}}{\longrightarrow} E_{1}^{p, 0}=\mathcal{E}^{p} .
$$

We have to check that $\alpha$ is a map of complexes. Since $\partial^{p, q, k}$ is zero for negative $k$, we only need to check that $\partial_{\mathcal{E}}^{p} \circ \bar{\alpha}^{p}=\partial^{p, 0,1} \circ \bar{\alpha}^{p+1}$. Now, $\partial_{\mathcal{E}}^{p}$ is the connecting map associated with (2.3.5.2), and therefore this is easy to verify (the diagram above might help).

We next define the isomorphism $\beta: \mathcal{E}_{\mathcal{F}} \stackrel{\sim}{\longrightarrow} \mathcal{F} \otimes_{Y} \mathrm{R}^{d} \pi_{*} \omega_{\pi}$. Let

$$
\mathbb{E}_{\pi}(\mathcal{F}):=E_{\Delta^{\prime}}\left(\pi^{*} \mathcal{F} \otimes\left(\omega_{\pi}[d]\right)\right) .
$$

Consider the diagram of complexes

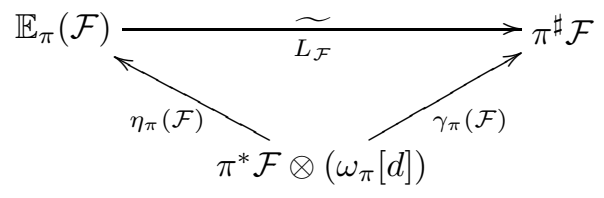

where $\eta_{\pi}(\mathcal{F})$ is the map in $[\mathbf{L N S} \S \S 10.1,(87)], L=L_{\mathcal{F}}$ the isomorphism of Cousin complexes is LNS $\S \S 8.1]$ (see second paragraph of loc.cit.) and $\gamma_{\pi}(\mathcal{F})$ is the 
composite $L \circ \eta_{\pi}(\mathcal{F})$. By definition of $\gamma_{\pi}$ the above diagram commutes. Some notations become necessary at this point. To that end, for every pair of integers $p<q$ define

$$
\begin{aligned}
\mathcal{F}_{p, q} & :=\sigma_{\geq p} \mathcal{F} / \sigma_{\geq q} \mathcal{F}, \\
\mathcal{A}_{p} & :=\pi^{*} \mathcal{F}_{p, p+1} \otimes\left(\omega_{\pi}[d]\right)=\left(\pi^{*} \mathcal{F} \otimes \omega_{\pi}\right)[d-p], \\
\mathcal{B}_{p} & :=\pi^{*}\left(\mathcal{F}_{p, p+2}\right) \otimes\left(\omega_{\pi}[d]\right) .
\end{aligned}
$$

Note that $\pi^{\sharp} \mathcal{F}_{p, q}=F^{p} / F^{q}$ [LNS $\left.\S \S 10.2,(89)\right]$. The map $\eta_{\pi}(\mathcal{F})$ is functorial in $\mathcal{F} \in \mathrm{Coz}_{\Delta}(\mathcal{y})$ as is $L_{\mathcal{F}}$. Therefore $\gamma_{\pi}(\mathcal{F})$ is also functorial in $\mathcal{F}$. We therefore have a commutative diagram of complexes of $\mathcal{O}_{\mathbb{P}}$-modules with exact rows

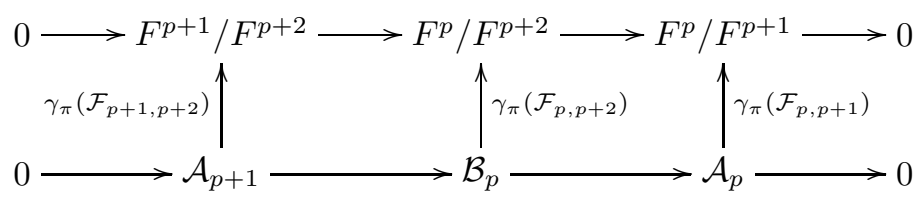

Denote the exact row on the top $E_{t}$ and the one at the bottom $E_{b}$. Now, by $\mathbf{L N S}$, Prop. 10.1.6], $\eta_{\pi}$ is a quasi-isomorphism and hence so is $\gamma_{\pi}$. On applying $\mathbf{R} \pi_{*}$ to the above diagram and identifying $\mathbf{R} \pi_{*} E_{t}$ with $\pi_{*} E_{t}$ (for $E_{t}$ consists of flasque complexes) we get a map (in fact an isomorphism) of triangles in $\mathbf{D}_{\mathrm{qc}}(Y)$ :

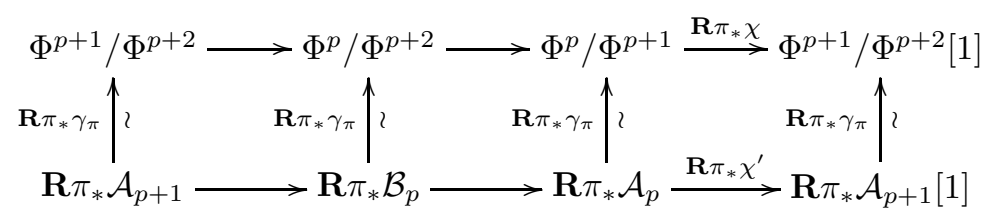

where $\chi: F^{p} / F^{p+1} \rightarrow F^{p+1} / F^{p+2}[1]$ is the map in $\mathbf{D}_{\mathrm{qc}}(\mathbb{P})$ arising from the standard triangle associated to the exact sequence $E_{t}$ and $\chi: \mathcal{A}_{p} \rightarrow \mathcal{A}_{p+1}[1]$ comes from a similar process involving $E_{b}$. We point out that the top triangle is the standard triangle associated to the exact sequence 2.3.5.2.

As a map of graded $\mathcal{O}_{Y}$-modules $\beta$ is defined in degree $p$ as the composite

$$
\begin{aligned}
\mathcal{E}^{p}=\mathrm{H}^{p}\left(\Phi^{p} / \Phi^{p+1}\right) \stackrel{\mathrm{H}^{p}\left(\mathbf{R} \pi_{*} \gamma_{\pi}\right)^{-1}}{\longrightarrow} & \mathrm{R}^{d} \pi_{*}\left(\pi^{*} \mathcal{F}^{p} \omega_{\pi}\right) \\
& \sim \mathcal{F}^{p} \otimes \mathrm{R}^{d} \pi_{*} \omega_{\pi} .
\end{aligned}
$$

We argue as follows to show that $\beta$ is a map of complexes. First, it is easy to see that

$$
\mathrm{H}^{p}\left(\mathbf{R} \pi_{*} \chi^{\prime}\right): \mathrm{R}^{d} \pi_{*}\left(\mathcal{F}^{p} \otimes \omega_{\pi}\right) \rightarrow \mathrm{R}^{d} \pi_{*}\left(\mathcal{F}^{p+1} \otimes \omega_{\pi}\right)
$$

is the negative of the map induced by $\pi^{*} \partial_{\mathcal{F}}^{p} \otimes 1: \pi^{*} \mathcal{F}^{p} \otimes \omega_{\pi} \rightarrow \pi^{*} \mathcal{F}^{p+1} \otimes \omega_{\pi}$. On the other hand $\mathrm{H}^{p}\left(\mathbf{R} \pi_{*} \chi\right)$ is the negative of the connecting map $\mathcal{E}^{p}=\mathrm{H}^{p}\left(\Phi^{p} / \Phi^{p+1}\right) \rightarrow$ $\mathrm{H}^{p+1}\left(\Phi^{p+1} / \Phi^{p+2}\right)=\mathcal{E}^{p+1}$. It is now clear from the definition of $\partial_{\mathcal{E}}^{\bullet}$ that $\beta$ is a map - in fact an isomorphism - of complexes.

It remains to show that the diagram (2.3.5.1) commutes. To do so, it is enough to assume that $Y$ is affine and and

$$
\mathcal{F}=i_{y} \Gamma_{y} \mathcal{F}
$$

for some $y \in Y$. The last assumption is equivalent to the assumption that

$$
\mathcal{F}=i_{y} \mathcal{F}(y)[-p]
$$


where $p=\Delta(y)$. Let

$$
M=\mathcal{F}(y), \quad \mathcal{G}=i_{y} M
$$

and let $x$ be a point on $\mathbb{P}$ which is closed in the fiber of $\pi$ over $y$. Let $R$ be the completion of $\mathcal{O}_{Y}$ at $y, S$ the completion of $\mathcal{O}_{\mathbb{P}}$ at $x$, and $\varphi: R \rightarrow S$ the map induced by $\pi$. We have a map

$$
\bar{\alpha}(x):\left(\pi^{\sharp} \mathcal{F}\right)(x) \longrightarrow \Gamma\left(\mathbb{P}, \mathcal{E}^{p}\right)
$$

defined as the composite

$$
\left(\pi^{\sharp} \mathcal{F}\right)(x) \stackrel{\text { natural }}{\longrightarrow} \Gamma\left(\mathbb{P}, \mathcal{A}^{p, 0}\right) \stackrel{2.3 .5 .3}{\longrightarrow} \Gamma\left(\mathbb{P}, \mathcal{E}^{p}\right) .
$$

In view of Proposition 2.3.4 it is enough to prove that the dotted arrow in the diagram

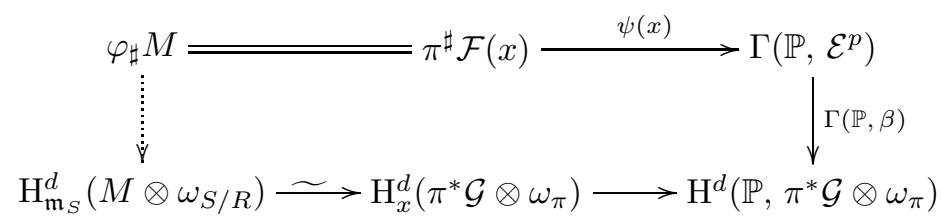

can be filled by (2.3.2) to make it commute. To that end consider the (possibly different) arrow $u: \varphi_{\sharp} M \stackrel{\sim}{\longrightarrow} \mathrm{H}_{\mathfrak{m}_{S}}^{d}\left(M \otimes \omega_{S / R}\right)$ described as follows. First we have a composite of maps of complexes

$$
\begin{aligned}
& \Gamma_{x} \pi^{\sharp} \mathcal{F} \stackrel{\sim}{\longrightarrow} \mathbf{R} \Gamma_{x} \pi^{\sharp} \mathcal{F} \underset{Q_{\mathbb{P}}\left(\gamma_{\pi}\right)^{-1}}{\sim} \mathbf{R} \Gamma_{x}\left(\mathcal{G}[-p] \otimes\left(\omega_{\pi}[d]\right)\right) \\
& =\quad \mathbf{R} \Gamma_{x}\left(\left(\mathcal{G} \otimes \omega_{\pi}\right)[d-p]\right) \\
& \stackrel{\sim}{\longrightarrow} \mathbf{R} \Gamma_{\mathfrak{m}_{S}}\left(\left(M \otimes \omega_{S / R}\right)[d-p]\right) \text {. }
\end{aligned}
$$

The map $u$ is defined as the $p$-th cohomology of this composite. In view of the definitions of the maps $\bar{\alpha}(x)$ and $\beta$, it is clear that 2.3.5.5) commutes when the dotted arrow is filled by $u$. Therefore we need to show that

$$
u=(2.3 .2) \text {. }
$$

To that end let

$$
u^{\prime}: \mathbb{E}_{\pi}(\mathcal{F})(x) \stackrel{\sim}{\longrightarrow} \mathrm{H}_{\mathfrak{m}_{S}}^{d}\left(M \otimes \omega_{S / R}\right)
$$

be defined in a manner completely analogous to the way $u$ was defined; i.e., $u^{\prime}$ is the $p$-th cohomology of the composite

$$
\begin{aligned}
& \Gamma_{x} \mathbb{E}_{\pi}(\mathcal{F}) \stackrel{\sim}{\longrightarrow} \mathbf{R} \Gamma_{x} \mathbb{E}_{\pi}(\mathcal{F}) \underset{Q_{\mathbb{P}}\left(\eta_{\pi}\right)^{-1}}{\sim} \mathbf{R} \Gamma_{x}\left(\mathcal{G}[-p] \otimes\left(\omega_{\pi}[d]\right)\right) \\
& =\quad \mathbf{R} \Gamma_{x}\left(\left(\mathcal{G} \otimes \omega_{\pi}\right)[d-p]\right) \\
& \stackrel{\sim}{\longrightarrow} \mathbf{R} \Gamma_{\mathfrak{m}_{S}}\left(\left(M \otimes \omega_{S / R}\right)[d-p]\right) .
\end{aligned}
$$

Clearly, by definition of $\eta_{\pi}, \gamma_{\pi}$ and $L$ we have

$$
u^{\prime}=u \circ L(x) .
$$

Now according to the discussion in [LNS Remark 10.1.10] we have

$$
u^{\prime}=(-1)^{\varepsilon(x)}(2.3 .2) \circ L(x)
$$

where $\varepsilon(x)=\Delta^{\prime}(x) \Delta(y)+\Delta^{\prime}(x)$. Since $x$ is closed in its fiber over $y$ (so that $\left.\Delta^{\prime}(x)=\Delta(y)\right)$ it is clear that $\varepsilon(x)$ is even. It follows that $u=(2.3 .2$. 
2.4. The Trace Theorem. Now that we have the Trace Theorem for relative projective space, the road to the general Trace Theorem is clear. We begin with a lemma which helps us reduce the problem to proper maps between ordinary schemes.

Lemma 2.4.1. Let $f:\left(X, \Delta^{\prime}\right) \rightarrow(\mathcal{Y}, \Delta)$ be a pseudo-proper map in $\mathbb{F}_{c}$ and let $\mathcal{F} \in \mathrm{Coz}_{\Delta}(\mathrm{y})$. Let $\mathcal{J} \in \mathcal{O}_{x}$ be a coherent ideal contained in the maximal defining ideal of $X$. For each positive integer $n$, let $v_{n}: X_{n} \hookrightarrow X$ be the closed immersion defined by $\mathrm{g}^{n}$ and let $f_{n}: \mathcal{X}_{n} \rightarrow \mathrm{y}$ be the composite $f_{n}=f v_{n}$. Then $\operatorname{Tr}_{f}(\mathcal{F})$ is a map of complexes if and only if $\operatorname{Tr}_{f_{n}}(\mathcal{F})$ is a map of complexes for every $n$.

Proof. Let $\mathcal{C}_{n}=\mathcal{H o m}_{x}^{\bullet}\left(v_{n *} \mathcal{O}_{x_{n}}, f^{\sharp} \mathcal{F}\right)$. We regard $\mathcal{C}_{n}$ as a subcomplex of $f^{\sharp} \mathcal{F}$. Note that $f^{\sharp} \mathcal{F}$ is the union (direct limit) of $\left\{\mathcal{C}_{n}\right\}$. If $\operatorname{Tr}_{f_{n}}(\mathcal{F})$ is a map of complexes, by Lemma 2.2.8 and (2.2.6),

$$
\left.\operatorname{Tr}_{f}(\mathcal{F})\right|_{\mathcal{C}_{n}}: \mathcal{C}_{n} \rightarrow \mathcal{F}
$$

is a map of complexes. Taking direct limits over $n$ we conclude that $\operatorname{Tr}_{f}(\mathcal{F})$ is a map of complexes. Conversely, if $\operatorname{Tr}_{f}(\mathcal{F})$ is a map of complexes, then (2.2.6) and Lemma 2.2.8 give the result.

Theorem 2.4.2. Suppose $f:\left(X, \Delta^{\prime}\right) \rightarrow(y, \Delta)$ is a pseudo-proper map in $\mathbb{F}_{c}$ and $\mathcal{F}$ is an object of $\mathrm{Coz}_{\Delta}(\mathrm{y})$.

(a) (Trace Theorem) The map

$$
\operatorname{Tr}_{f}(\mathcal{F}): f_{*} f^{\sharp} \mathcal{F} \rightarrow \mathcal{F}
$$

is a map of complexes which is functorial in $\mathcal{F}$.

(b) (Transitivity of Traces) If $g:\left(\mathcal{W}, \Delta^{\prime \prime}\right) \rightarrow\left(X, \Delta^{\prime}\right)$ is a second pseudoproper map then

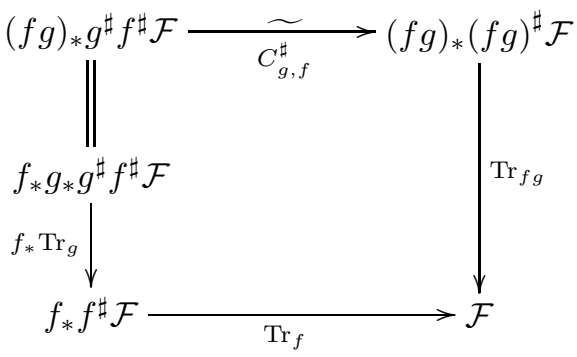

commutes.

Proof. Part (b) follows from Part (a) and (2.2.6). If $\mathcal{J}$ is a defining ideal of $X$, then the maps $f_{n}$ of Lemma 2.4.1 are adic, and hence by Lemma 2.4.1 we are reduced to the case where $f$ is proper.

Next consider a defining ideal $\mathcal{J}$ of $y$ and set $\mathcal{J}:=\mathfrak{J} \mathcal{O}_{x}$. By our assumption $\mathcal{J}$ is a defining ideal for $x$. Let $u_{n}: y_{n} \rightarrow Y$ be the closed subscheme of $y$ defined by $\mathcal{J}^{n}$ and $v_{n}: X_{n} \rightarrow X$ the closed subscheme of $\mathcal{J}$ defined by $\mathcal{J}^{n}$. Let $h_{n}$ be the induced map from $X_{n}$ to $Y_{n}$. If $\operatorname{Tr}_{h_{n}}\left(u_{n}^{\sharp} \mathcal{F}\right)$ is a map of complexes for each $n$, then by Lemma 2.2.8 applied to $u_{n}$ and by (2.2.6) we see that the Trace Theorem is true for $f_{n}=f v_{n}$ and hence by Lemma 2.4.1 it is true for $f$. Now $h_{n}$ is a proper map of ordinary schemes. Thus we are reduced to proving the Trace Theorem when $f$ is a proper map of ordinary schemes in $\mathbb{F}$. Since we have proved the Trace Theorem 
for relative projective space, the proof of [Hrt p.369, Theorem 2.1] applies mutatis mutandis and we are done.

\section{The twisted inverse image pseudofunctor}

In this section we summarize Nayak's work on the pseudofunctor -! on $\mathbb{G}$-laid out in $\mathbf{N a y}$ - which extends the definition of - ! given by Alonso, Jeremías and Lipman in AJL2 for pseudo-proper maps. Here are some hueristics to orient the reader. Notionally "upper shriek" is to be thought of as a right adjoint to the (perhaps non existent) derived "direct image with proper supports on torsion sheaves". This hueristic would dictate, for example, that we set $f^{!}$equal to $\mathbf{R} \Gamma_{x}^{\prime} f^{*}=f^{*} \mathbf{R} \Gamma_{y}^{\prime}$ for an open immersion $f: \mathcal{X} \rightarrow \mathcal{y}$ of noetherian formal schemes - for the corresponding direct image with proper supports "is" the functor "extension by zero". Since the objects of $\mathbb{G}$ are noetherian formal schemes, Deligne's definition of direct image with proper support (see his appendix to $[\mathbf{H r t}]$ ) does not apply principally because we cannot guarantee that a coherent ideal sheaf $\mathcal{J} \subset \mathcal{O}_{\mathfrak{U}}$ on an open subscheme $\mathcal{U}$ of a noetherian formal scheme $\mathcal{V}$ extends to a coherent ideal sheaf $\mathcal{J} \subset \mathcal{O}_{\mathcal{V}}$ on $\mathcal{V}$. This is, incidentally, the reason why Verdier's proof of the localness of - ! V p. 395, Corollary 1] does not apply mutatis mutandis to our situation ${ }^{6}$. Nayak establishes the localness result and pseudofunctoriality of - !. Here then is the promised summary.

3.1. Factorizations. For $f: X \rightarrow y$ in $\mathbb{G}$, we wish to define $f^{!}: \widetilde{\mathbf{D}}_{\mathrm{qc}}^{+}(y) \rightarrow$ $\left.\mathbf{D}_{\mathrm{qct}}^{+} X\right)$. If $f$ is pseudo-proper, $f^{!}$is defined to be the functor $f_{\mathbf{t}}^{\times} \mid \widetilde{\mathbf{D}}_{\mathrm{qc}}^{+}(y)$, where $f_{\mathbf{t}}^{\times}$is right adjoint to $\mathbf{R} f_{*}: \mathbf{D}_{\mathrm{qct}}(X) \rightarrow \mathbf{D}(y)$-guaranteed to exist by AJL2, Theorem 2(a)] and [AJL2 Theorem 6.1(a)] (see also the beginning of 1.3 of Ibid as well as the notation before Theorem 7.4 in Ibid). If $f$ is an open immersion, then in light of the above discussion, we set

$$
f^{!}:=\mathbf{R} \Gamma_{x}^{\prime} f^{*}\left(=\mathbf{R} f^{*} \Gamma_{y}^{\prime}\right) .
$$

If $f$ is a general map in $\mathbb{G}$, then $f$ can be written as a composite of open immersions and pseudo-proper maps (in any order), and the above gives us a clue on how to proceed. The problems that need to be addressed are compatibilities of different definitions corresponding to different factorizations of the same map. Since the issues involved impact this paper we elaborate.

A sequence $\boldsymbol{F}=\left(f_{1}, \ldots, f_{n}\right)$ of maps in $\mathbb{G}$ is called a factorization if $f_{1} \circ \ldots \circ f_{n}$ is defined (the maps are composable) and an individual $f_{i}$ in the sequence is either pseudo-proper or an open immersion. If $f=f_{1} \circ \ldots \circ f_{n}$, then $\boldsymbol{F}$ is called a factorization of $f$. We often write $|\boldsymbol{F}|=f_{1} \circ \ldots \circ f_{n}$. We define

$$
\boldsymbol{F}^{!}:=f_{n}^{!} \ldots f_{1}^{!} \text {. }
$$

If $\boldsymbol{F}=\left(f_{1}, \ldots, f_{n}\right)$ and $\boldsymbol{G}=\left(g_{1}, \ldots, g_{m}\right)$ are factorizations such that $f_{n} \circ g_{1}$ is defined then we set

$$
\boldsymbol{F} * \boldsymbol{G}:=\left(f_{1}, \ldots, f_{n}, g_{1}, \ldots, g_{m}\right) .
$$

Note that $\boldsymbol{F} * \boldsymbol{G}$ is a factorization of $|\boldsymbol{F}| \circ|\boldsymbol{G}|$ and that

$$
(\boldsymbol{F} * \boldsymbol{G})^{!}=\boldsymbol{G}^{!} \boldsymbol{F}^{!} .
$$

\footnotetext{
${ }^{6}$ The proof given in AJL2 Proposition 8.3.1] is incorrect. See however a partial resolution to the localness question in AJL3.
} 
In $\mathbf{N a y}$ Nayak proves that if $\boldsymbol{F}_{\mathbf{1}}$ and $\boldsymbol{F}_{\mathbf{2}}$ are two factorizations of $f$, then there is an isomorphism

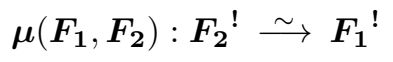

satisfying the following properties.

(a) $\boldsymbol{\mu}\left(\boldsymbol{F}_{\mathbf{1}}, \boldsymbol{F}_{\mathbf{1}}\right)=\mathbf{1}_{\boldsymbol{F}^{!}}$.

(b) If $\boldsymbol{F}_{\mathbf{3}}$ is a third factorization of $f$, then

$$
\boldsymbol{\mu}\left(\boldsymbol{F}_{1}, \boldsymbol{F}_{2}\right) \circ \boldsymbol{\mu}\left(\boldsymbol{F}_{2}, \boldsymbol{F}_{3}\right)=\boldsymbol{\mu}\left(\boldsymbol{F}_{1}, \boldsymbol{F}_{3}\right) .
$$

(c) If $\boldsymbol{F}$ is a factorization of $f, \boldsymbol{G}_{\mathbf{1}}, \boldsymbol{G}_{\mathbf{2}}$ of $g$ and $\boldsymbol{H}$ of $h$ where $g$ and $h$ are such that $f \circ g \circ h$ is defined, then setting $\boldsymbol{E}_{\mathbf{1}}=\boldsymbol{F} * \boldsymbol{G}_{\mathbf{1}} * \boldsymbol{H}$ and $\boldsymbol{E}_{\mathbf{2}}=\boldsymbol{F} * \boldsymbol{G}_{\mathbf{2}} * \boldsymbol{H}$, the isomorphism $\boldsymbol{\mu}\left(\boldsymbol{E}_{\mathbf{1}}, \boldsymbol{E}_{\mathbf{2}}\right)$ is induced by $\boldsymbol{\mu}\left(\boldsymbol{G}_{\mathbf{1}}, \boldsymbol{G}_{\mathbf{2}}\right)$, i.e.

$$
\boldsymbol{\mu}\left(\boldsymbol{E}_{\mathbf{1}}, \boldsymbol{E}_{\mathbf{2}}\right)=\boldsymbol{H}^{!}\left(\boldsymbol{\mu}\left(\boldsymbol{G}_{\mathbf{1}}, \boldsymbol{G}_{\mathbf{2}}\right)\left(\boldsymbol{F}^{!}\right)\right)
$$

(d) If

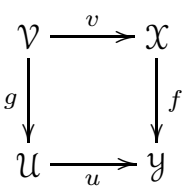

is cartesian with $f$ pseudo-proper and $u$ an open immersion, then the base change map $\beta: v^{*} f^{!} \stackrel{\sim}{\longrightarrow} g^{!} \Gamma_{\mathcal{u}}^{\prime} u^{*}=g^{!} u^{*}$ of AJL2 Theorem 7.4] (note that $u$ is adic) is given by

$$
\beta=\boldsymbol{\mu}\left(\boldsymbol{F}_{\mathbf{1}}, \boldsymbol{F}_{\mathbf{2}}\right)
$$

where $\boldsymbol{F}_{\mathbf{1}}=(u, g)$ and $\boldsymbol{F}_{\mathbf{2}}=(f, v)$.

Now for each map $f$ in $\mathbb{G}$ pick a distinguished factorization $f$ of $f$-with the understanding that if $f$ is either an open immersion or pseudo-proper $f=(f)$. Nayak then defines $f^{!}$as

$$
f^{!}:=f^{!} .
$$

In fact Nayak shows that $-!$ is a pseudofunctor ${ }^{7}$, the required isomorphism

$$
C_{f, g}^{!}: f^{!} g^{!} \stackrel{\sim}{\longrightarrow}(g f)^{!}
$$

being (with $h=g f$ )

$$
C_{f, g}^{!}:=\boldsymbol{\mu}(\boldsymbol{h}, \boldsymbol{g} * \boldsymbol{f}) .
$$

For a factorization $\boldsymbol{F}$ of $f$, we have a canonical isomorphism

$$
\nu_{\boldsymbol{F}}: \boldsymbol{F}^{!} \stackrel{\sim}{\longrightarrow} f^{!}
$$

given by $\nu_{\boldsymbol{F}}=\boldsymbol{\mu}(\boldsymbol{f}, \boldsymbol{F})$.

\footnotetext{
${ }^{7}$ More precisely a pre-pseudofunctor in the sense of Lipman's article in this volume. However if for each $X \in \mathbb{G}$ we set $X^{!}=\mathbf{D}_{\mathrm{qct}}^{+}(X)$ rather than $\widetilde{\mathbf{D}}_{\mathrm{qc}}^{+}(X)$, then $-!$ is a pseudofunctor.
} 
3.2. Flat base change. Suppose we have a cartesian square of noetherian formal schemes

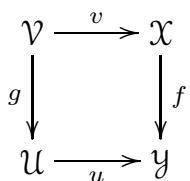

with $u$ flat. The flat base change theorem of Alonso, Jeremías and Lipman [AJL2, Theorem 7.4] states that if $f$ is pseudo-proper there is a base change isomorphism (between functors on $\widetilde{\mathbf{D}}_{\mathrm{qc}}^{+}(\mathrm{y})$ )

$$
\beta=\beta(f, u): \mathbf{R} \Gamma_{\mathcal{v}}^{\prime} v^{*} f^{!} \stackrel{\sim}{\longrightarrow} g^{!} \mathbf{R} \Gamma_{\mathcal{u}}^{\prime} u^{*} .
$$

Nayak extends $\beta$ to the case when $f$ is a composite of compactifiable maps. In greater detail, if $f$ is an open immersion, let $\beta(f, u)$ be the obvious isomorphism. For a general morphism $f$ in $\mathbb{G}$, suppose $\boldsymbol{F}$ is a factorization of $f$. Then $\boldsymbol{F}$ induces, via the base change map $u$, a factorization $\mathcal{U} \times y \boldsymbol{F}$ of $g$. Setting $\boldsymbol{G}=\mathcal{U} \times y \boldsymbol{F}$ one gets, by applying $\beta$ successively to the maps in the sequence $\boldsymbol{F}$, an isomorphism

$$
\beta(\boldsymbol{F}): \mathbf{R} \Gamma_{\mathcal{v}}^{\prime} v^{*} \boldsymbol{F}^{!} \stackrel{\sim}{\longrightarrow} \boldsymbol{G}^{!} \mathbf{R} \Gamma_{\mathcal{u}}^{\prime} u^{*} .
$$

This gives us an isomorphism

$$
\beta=\beta(f, u): \mathbf{R} \Gamma_{\mathcal{V}}^{\prime} v^{*} f^{!} \stackrel{\sim}{\longrightarrow} g^{!} \mathbf{R} \Gamma_{\mathcal{u}}^{\prime} u^{*}
$$

given by

$$
\beta=\boldsymbol{\mu}(\boldsymbol{g}, \mathcal{U} \times \boldsymbol{y} \boldsymbol{f})\left(\mathbf{R} \Gamma_{\mathfrak{U}}^{\prime} u^{*}\right) \circ \beta(\boldsymbol{f}) .
$$

Nayak shows that the map $\beta$ is "independent" of the chosen distinguished factorization $\boldsymbol{f}$ of $f$ in the following sense. If $\boldsymbol{F}$ is any factorization of $f$ and $\boldsymbol{G}=\mathcal{U} \times y \boldsymbol{F}$, then the diagram

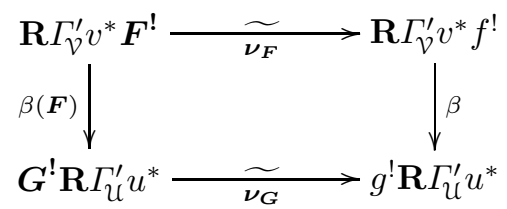

commutes.

3.3. Comparing pseudofunctors. The construction of $-!$ on $\mathbb{G}$ is built on its description on open immersions, pseudo-proper maps and the fact that it satisfies a compatibility relationship for open base changes of pseudo-proper maps. This allows one to compare a pseudofunctor on a subcategory of $\mathbb{G}$ with $\_$! if we have a comparison for pseudo-proper maps and for open immersions, and certain compatibility relations. We give more details below.

Let $\overline{\mathbb{G}}$ be a full subcategory of $\mathbb{G}$ such that if $y \in \overline{\mathbb{G}}$ and $f: x \rightarrow y$ is a map in $\mathbb{G}$, then $f$ is a map in $\overline{\mathbb{G}}($ i.e. $X \in \overline{\mathbb{G}})$. Note that all open subschemes and all closed subschemes of $y$ are in $\overline{\mathbb{G}}$, and if $g: \mathcal{W} \rightarrow y$ is another map in $\overline{\mathbb{G}}$, then $x \times y \mathcal{W} \in \overline{\mathbb{G}}$. Note also that $\mathbb{F}^{*}$ and $\mathbb{F}^{*} \cap \mathbb{F}^{r}$ provide examples of $\overline{\mathbb{G}}$.

Let $\mathbf{P}$ be the class of pseudo-proper maps in $\overline{\mathbb{G}}$ and $\mathbf{F}$ the class of open immersions in $\overline{\mathbb{G}}$. Suppose we have another pseudofunctor $-^{\natural}$ on $\overline{\mathbb{G}}$ such that for each object $X$ in $\overline{\mathbb{G}}$ we have a functor

$$
S_{x}: x^{\natural} \longrightarrow x^{!}
$$


and for each map $f: X \rightarrow y$ in $\mathbf{P} \cup \mathbf{F}$ a functorial map

$$
\gamma_{f}: S_{x} f^{\sharp} \longrightarrow f^{!} S_{y}
$$

such that

(a) If $f \in \mathbf{F}, \gamma_{f}$ is an isomorphism.

(b) If $X \stackrel{f}{\rightarrow} y \stackrel{g}{\rightarrow} z$ are a pair of maps such that either both $f$ and $g$ are in $\mathbf{P}$ or both $f$ and $g$ are in $\mathbf{F}$, then the diagram

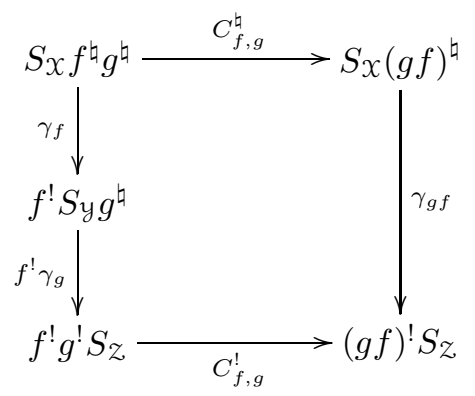

commutes.

(c) If

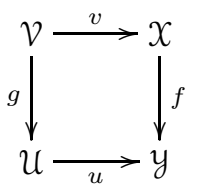

is cartesian with $u \in \mathbf{F}$ and $f \in \mathbf{P}$, then the following diagram commutes

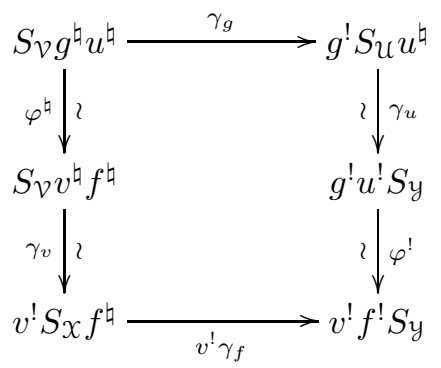

where $\varphi^{\natural}=\left(C_{v, f}^{\natural}\right)^{-1} C_{g, u}^{\natural}$ and $\varphi^{!}=\left(C_{v, f}^{!}\right)^{-1} C_{g, u}^{!}$.

In Nay, Nayak proves

TheOREM 3.3.4. (Nayak) Under the above hypotheses on $\left(-^{\natural}, S, \gamma\right)$, the association $f \mapsto \gamma_{f}, f \in \mathbf{P} \cup \mathbf{F}$, can be extended uniquely to all morphisms

$$
f: x \rightarrow y
$$

in $\overline{\mathbb{G}}$ in such a way that

(a) If $y \stackrel{g}{\rightarrow} z$ is a second map in $\overline{\mathbb{G}}$, then the diagram (3.3.1) commutes.

(b) The natural transformation $\gamma_{f}$ is compatible with open immersions into $y$, i.e., whenever we have a cartesian square (3.3.2) with $u \in \mathbf{F}$ and $f$ any map in $\overline{\mathbb{G}}$ the diagram (3.3.3) commutes. 
Note that the uniqueness assertion is clear. Indeed if $\boldsymbol{F}=\left(f_{1}, \ldots, f_{n}\right)$ is any factorization of a map $f: x \rightarrow y$ in $\overline{\mathbb{G}}$, and

$$
\boldsymbol{F}^{\natural}:=f_{1}^{\natural} \ldots f_{n}^{\natural}
$$

then we have a map

$$
\gamma(\boldsymbol{F}): S_{x} \boldsymbol{F}^{\natural} \rightarrow \boldsymbol{F}^{!} S_{y}
$$

given by the composite (where we are suppressing the functors $S_{x}$ etc.)

$$
\begin{aligned}
& \boldsymbol{F}^{\natural}=f_{n}{ }^{\natural} \ldots f_{1}{ }^{\natural} \stackrel{\gamma_{f_{n}}}{\longrightarrow} f_{n}^{!} f_{n-1}^{\natural} \ldots f_{1}^{\natural} \\
& \stackrel{f_{n}^{!} \gamma_{f_{n-1}}}{\longrightarrow} f_{n}^{!} f_{n-1}^{!} f_{n-2}{ }^{\natural} \ldots f_{1}^{\natural} \\
& \ldots \\
& \stackrel{f_{n}^{!} \ldots f_{2}^{!} \gamma_{f_{1}}}{\longrightarrow} f_{n}^{!} \ldots f_{1}^{!}=\boldsymbol{F}^{!},
\end{aligned}
$$

and $\gamma_{f}$ is characterized by the commutativity of

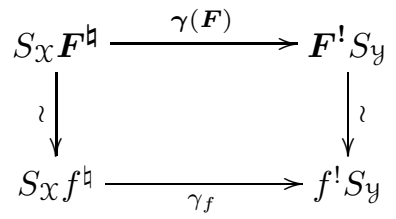

where the downward arrows are the natural ones obtained from $-^{\natural}$ and $-^{!}$.

\section{The comparison map}

In this section we define the fundamental comparison map which serves as the bridge between the pseudofunctors $-\sharp$ and - ! (see Definition 4.1.4.1). This comparison is easily defined when $f$ is pseudo-proper, but has a more involved definition for a general $f$ in $\mathbb{F}_{c}^{*}$.

4.1. Pseudo-proper maps. Suppose $f:\left(X, \Delta^{\prime}\right) \rightarrow(y, \Delta)$ is a pseudo-proper map in $\mathbb{F}_{c}$. For $\mathcal{F} \in \operatorname{Coz}(y)$, the trace map

$$
\operatorname{Tr}_{f}(\mathcal{F}): f_{*} f^{\sharp} \mathcal{F} \longrightarrow \mathcal{F}
$$

gives, on applying $Q_{y}$, a map

$$
Q_{y}\left(\operatorname{Tr}_{f}(\mathcal{F})\right): \mathbf{R} f_{*} Q x\left(f^{\sharp} \mathcal{F}\right) \rightarrow Q_{y}(\mathcal{F})
$$

whence we have, by the universal property of $\left(f^{!}, \tau_{f}\right)$, a unique map $\gamma_{f}^{!}(\mathcal{F})$ : $Q_{x} f^{\sharp} \mathcal{F} \rightarrow f^{!} Q_{y} \mathcal{F}$ such that $Q_{y} \operatorname{Tr}_{f}(\mathcal{F})=\tau_{f} \mathbf{R} f_{*}\left(\gamma_{f}^{!}(\mathcal{F})\right)$. This map is functorial in $\mathcal{F}$ giving a comparison map of functors

$$
\gamma_{f}^{!}: Q_{x} f^{\sharp} \longrightarrow f^{!} Q_{y} .
$$

Note that the source of the functors (being compared by $\gamma_{f}^{!}$) is $\operatorname{Coz}_{\Delta}(y)$ and the target is $\mathbf{D}_{\text {qct }}^{+}(X)$. We would like to extend the definition of $\gamma_{f}^{!}$to the case where $f \in \mathbb{F}_{c}^{*}$ (i.e. $f$ is a composite of pseudo-proper maps and open immersions in any order). We will, from now on, often suppress the localization functors $Q_{x}, Q_{y}$ etc..

We begin with two Lemmas. Let $f$ be as above (i.e. pseudo-proper and in $\mathbb{F}_{c}$ ). Let $u: \mathcal{U} \rightarrow \mathcal{Y}$ be an open immersion, $\mathcal{V}=\mathcal{U} \times y \mathcal{X}, v: \mathcal{V} \rightarrow X$ and $g: \mathcal{V} \rightarrow \mathcal{U}$ the two projections. We have -from the local nature of direct images- $u^{*} \mathbf{R} f_{*}=\mathbf{R} g_{*} v^{*}$. The sheafified version of duality on formal schemes [AJL2, Theorem 8.2] gives us 
$v^{*} f^{!} \mathcal{F}=g^{!} u^{*} \mathcal{F}$ and $u^{*} \tau_{f}(\mathcal{F})=\tau_{g}\left(u^{*} \mathcal{F}\right)$ for $\mathcal{F} \in \widetilde{\mathbf{D}}_{\mathrm{qc}}^{+}(\mathrm{y})$. We also have, by the construction of $f^{\sharp}$ and $g^{\sharp}, v^{*} f^{\sharp}=g^{\sharp} u^{*}$.

LEMma 4.1.2. In the above situation the diagram

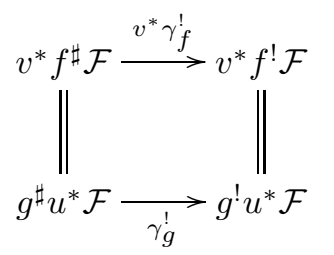

commutes for $\mathcal{F} \in \operatorname{Coz}(X)$.

Proof. From the punctual nature of the definition of $\operatorname{Tr}_{f}$ it is clear that $\operatorname{Tr}_{f}$ behaves well with respect to open immersions into $y$, i.e. $u^{*} \operatorname{Tr}_{f}(\mathcal{F})=\operatorname{Tr}_{g}\left(u^{*} \mathcal{F}\right)$. The Lemma is a simple consequence of this. (See also Proposition 4.2 .2 where a more general result is proved somewhat elaborately.)

LEMMA 4.1.3. Let $\left(X, \Delta_{1}\right) \stackrel{f}{\rightarrow}\left(y, \Delta_{2}\right) \stackrel{g}{\rightarrow}\left(\mathcal{Z}, \Delta_{3}\right)$ be a pair of pseudo-proper maps in $\mathbb{F}_{c}$. Then the diagram

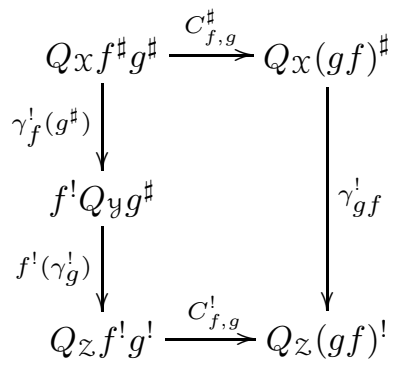

commutes.

Proof. We will suppress the symbols $Q x$ and $Q_{y}$. By the universal property of $\left((g f)^{!}, \tau_{g f}\right)$ it is enough to show

$$
\tau_{g f} \circ \mathbf{R}(g f)_{*}\left(c_{g, f}^{!} \circ f^{!}\left(\gamma_{g}^{!}\right) \circ \gamma_{f}^{!}\left(g^{\sharp}\right)\right)=\tau_{g f} \circ \mathbf{R}(g f)_{*}\left(\gamma_{g f}^{!} \circ C_{f, g}^{\sharp}\right)
$$

In what follows, we save on notation by writing $f_{*}, g_{*}$ and $(g f)_{*}$ for $\mathbf{R} f_{*}, \mathbf{R} g_{*}$ and $\mathbf{R}(g f)_{*}$.

Consider the following diagram

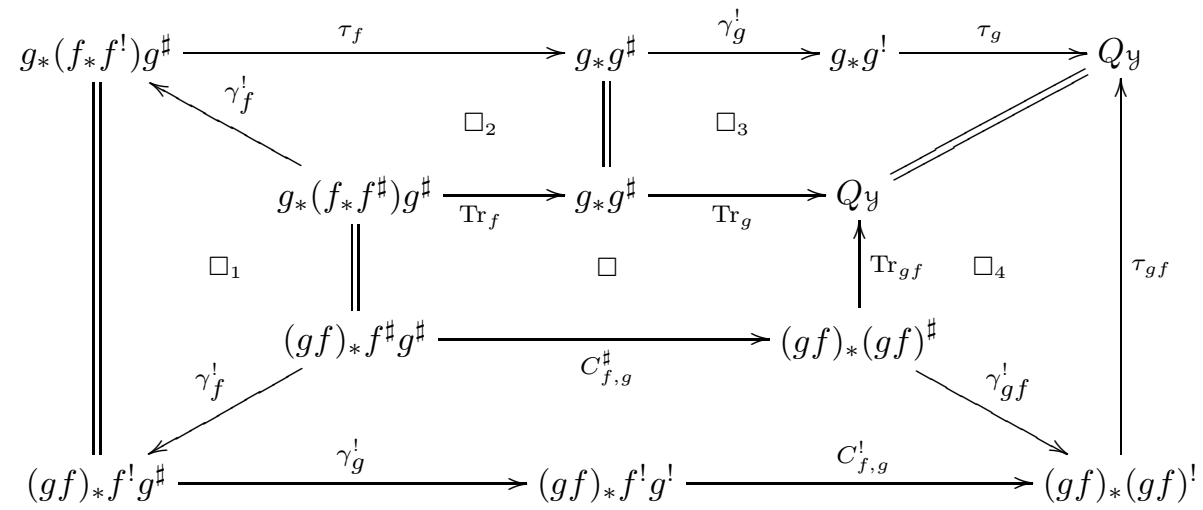


If we can show that $\square_{1}, \square_{2}, \square_{3}, \square_{4}, \square$ and the outer rectangle commute then (4.1.3.1) follows easily. Now $\square_{1}, \ldots, \square_{4}$ and $\square$ clearly commute ( $\square$ commutes by the transitivity of the trace). It remains to show that the outer rectangle commutes. To that end consider the diagram

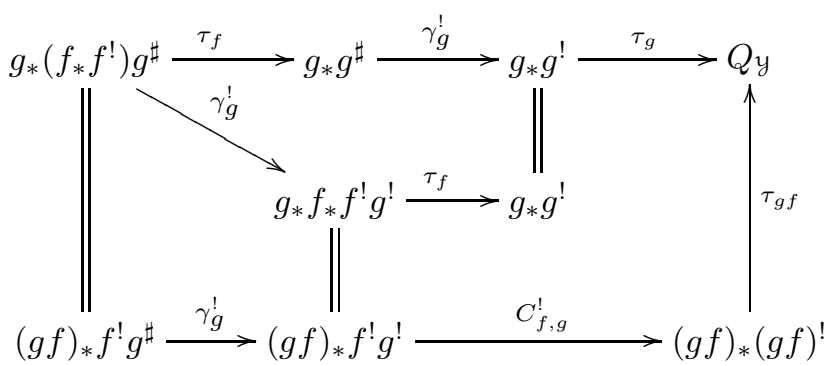

The subdiagram on the left obviously commutes. The one on top commutes by the functoriality of $\gamma_{g}^{!}$and $\tau_{f}$. The remaining (L-shaped) subdiagram commutes by the definition of $C_{f, g}^{!}$.

We are now in a position to prove

THEOREM 4.1.4. There exists a unique family of natural transformations

$$
\gamma_{f}^{!}: Q_{x} f^{\sharp} \longrightarrow f^{!} Q_{y},
$$

one for each morphism $f:\left(X, \Delta^{\prime}\right) \rightarrow(y, \Delta)$ in $\mathbb{F}_{c}^{*}$ such that

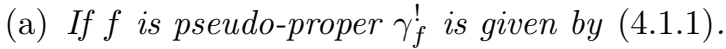

(b) If $f$ is an open immersion then $\gamma_{f}^{!}$is the identity transformation on $f^{*}$.

(c) The map $\gamma_{f}^{!}$is compatible with open immersions into $y$, i.e. Lemma 4.1.2 holds with the weaker hypothesis that $f \in \mathbb{F}_{c}^{*}$.

(d) Lemma 4.1.3 holds under the weaker hypothesis that $f$ and $g$ are in $\mathbb{F}_{c}^{*}$.

Proof. Let the triple $\left(\overline{\mathbb{G}},\left\{S_{X}\right\},\left\{\gamma_{f}\right\}_{\mathbf{P} \cup \mathbf{F}}\right)$ in Subsection 3.3 be given by the data $\left(\mathbb{F}_{c}^{*},\left\{Q_{X}\right\},\left\{\gamma_{f}^{!}\right\}_{\mathbf{P} \cup \mathbf{F}}\right)$. By Lemma 4.1.2 and Lemma 4.1.3 we see that Theorem 3.3.4 applies to our situation.

REMARK 4.1.5. We remind the reader how the map $\gamma_{f}^{!}$is computed in practice for $f: X \rightarrow Y$ a general maps in $\mathbb{F}_{c}^{*}$. First, if $f$ is an open immersion, then recall that $f^{!}=f^{*}$ and $f^{\sharp}=f^{*} \mid \operatorname{Coz}(X)$, and so we set $\gamma_{f}^{!}$equal to the identity map $\left.\left.f^{*}\right|_{\mathrm{Coz}(y)} \rightarrow f^{*}\right|_{\mathrm{Coz}(y)}$. For general $f \in \mathbb{F}_{c}^{*}$ we proceed as follows: if $\boldsymbol{F}=\left(f_{1}, \ldots, f_{n}\right)$ is a factorization of $f$, we write $\boldsymbol{F}^{\sharp}=f_{n}^{\sharp} \ldots f_{1}^{\sharp}$. We define

$$
\gamma^{!}(\boldsymbol{F}): \boldsymbol{F}^{\sharp} \longrightarrow \boldsymbol{F}^{!}
$$

as the composite

$$
\begin{gathered}
\boldsymbol{F}^{\sharp}=f_{n}^{\sharp} \ldots f_{1}^{\sharp} \stackrel{\gamma_{f_{n}}^{!}}{\stackrel{f_{n}^{!} \gamma_{f_{n-1}}^{!}}{\longrightarrow} f_{n}^{!} f_{n-1}^{\sharp} \ldots f_{1}^{\sharp}} f_{n}^{!} f_{n-1}^{!} f_{n-2}^{\sharp} \ldots f_{1}^{\sharp} \\
\vdots \\
\stackrel{f_{n}^{!} \ldots f_{2}^{!} \gamma_{f_{1}}^{!}}{\longrightarrow} f_{n}^{!} \ldots f_{1}^{!}=\boldsymbol{F}^{!} .
\end{gathered}
$$


Then

$$
\gamma_{f}^{!}: f^{\sharp} \rightarrow f^{!}
$$

is characterized by the commutativity of

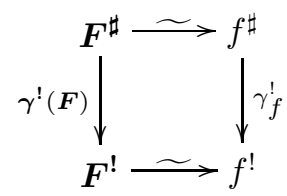

4.2. Étale base change. Suppose we have a cartesian square

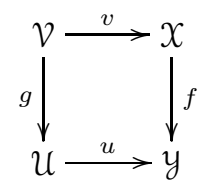

with $u$ (and hence $v$ ) flat and adic. If $\mathcal{F} \in \mathbf{D}_{\mathrm{qct}}^{+}(\mathcal{y})$ and $\mathcal{G} \in \in \mathbf{D}_{\mathrm{qct}}^{+}(\mathcal{X})$, then $\mathbf{R} \Gamma_{\mathcal{u}}^{\prime} u^{*} \mathcal{F}=u^{*} \mathbf{R} \Gamma_{y}^{\prime} \mathcal{F}=u^{*} \mathcal{F}$ and $\mathbf{R} \Gamma_{v}^{\prime} v^{*} \mathcal{G}=v^{*} \Gamma_{x}^{\prime} \mathcal{G}=v^{*} \mathcal{G}$ (see AJL2 Corollary 5.2.11(c)]). By (3.2.1) we have a base change isomorphism

$$
\mathbf{R} \Gamma_{\mathcal{V}}^{\prime} v^{*} f^{!} \stackrel{\sim}{\longrightarrow} g^{!} \mathbf{R} \Gamma_{\mathcal{u}}^{\prime} u^{*}
$$

and in light of the relations we have given (in our special case), this translates to a functorial isomorphism

$$
\beta_{\mathcal{F}}: v^{*} f^{!} \mathcal{F} \stackrel{\sim}{\longrightarrow} g^{!} u^{*} \mathcal{F} \quad\left(\mathcal{F} \in \mathbf{D}_{\text {qct }}^{+}(y)\right) .
$$

If $f$ is pseudo-proper the map $\beta_{\mathcal{F}}$ is obtained (when, as we have assumed, $u$ is adic) in the following manner. By AJL2, Proposition 7.2(b)] we have a functorial isomorphism

$$
\theta_{\mathcal{G}}: u^{*} \mathbf{R} f_{*} \mathcal{G} \stackrel{\sim}{\sim} \mathbf{R} g_{*} v^{*} \mathcal{G} \quad(\mathcal{G} \in \mathbf{D}(X))
$$

where the map $\theta_{\mathcal{G}}$ is adjoint to the composite

$$
\mathbf{R} f_{*} \mathcal{G} \rightarrow \mathbf{R} f_{*} \mathbf{R} v_{*} v^{*} \mathcal{G} \rightarrow \mathbf{R} u_{*} \mathbf{R} g_{*} v^{*} \mathcal{G} .
$$

The map $\beta_{\mathcal{G}}$ is then defined via the universal property of $\left(g^{!}, \tau_{g}\right)$ as the map adjoint to the composite

$$
\mathbf{R} g_{*} v^{*} f^{!} \mathcal{F} \stackrel{\theta_{f^{\prime} \mathcal{F}}^{-1}}{\longrightarrow} u^{*} \mathbf{R} f_{*} f^{!} \mathcal{F} \stackrel{u^{*} \tau_{f}}{\longrightarrow} u^{*} \mathcal{F}
$$

The following proposition relates the above base change with a base change for the pseudofunctor $-\sharp$ in a special situation. We treat the isomorphisms in LNS, Prop. 10.3.6,(90),(91)] as equalities.

Proposition 4.2.2. Suppose $f:\left(\mathcal{X}, \Delta^{\prime}\right) \rightarrow(\mathcal{Y}, \Delta)$ is a map in $\mathbb{F}_{c}^{*}$, and $u: \mathcal{U} \rightarrow$ $y$ ia an étale adic map such that for $q \in \mathcal{U}$ the natural map $\widehat{\mathcal{O}}_{y, u(q)} \rightarrow \widehat{\mathcal{O}}_{\mathcal{u}, q}$ is an isomorphism (equivalently, the residue fields at $u(q)$ and $q$ are isomorphic via the natural map between them). Consider the cartesian square (4.2.1) above. Then for 
$\mathcal{F} \in \operatorname{Coz}(y)$ the following diagram commutes

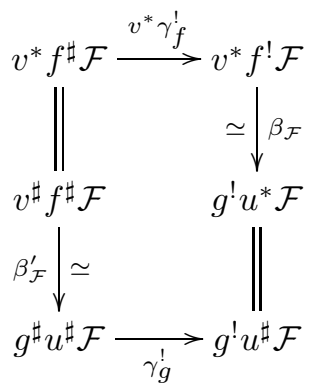

where $\beta_{\mathcal{F}}^{\prime}: v^{\sharp} f^{\sharp} \mathcal{F} \stackrel{\sim}{\longrightarrow} g^{\sharp} f^{\sharp} \mathcal{F}$ is the functorial isomorphism arising from the pseudofunctorial nature of $\mathbf{-}$, and the equalities are the identifications given in LNS. Prop. 10.3.6, (90), (91)].

Proof. The proposition is trivially true if $f$ is an open immersion, for, in this case, $\gamma_{f}^{!}: f^{*} \rightarrow f^{*}$ and $\gamma_{g}^{!}: g^{*} \rightarrow g^{*}$ are the identity maps.

Suppose the proposition is true when $f$ is pseudo-proper. We claim that then it is true for $f$ a composite of pseudo-proper and open maps. To see this, fix $f$ in $\mathbb{F}_{c}^{*}$. Suppose $\boldsymbol{F}=\left(f_{1}, \ldots, f_{n}\right)$ is a factorization of $f$. Recall that we require each $f_{i}$ to be either pseudo-proper or an open immersion. The map $u: \mathcal{U} \rightarrow \mathcal{V}$ induces, via base change, a factorization $\boldsymbol{G}=\left(g_{1}, \ldots, g_{n}\right)$ of $g$-the factorization $\mathcal{U} \times \boldsymbol{y} \boldsymbol{F}$ in Section 3 such that with $u=v_{0}, v=v_{n}, \mathcal{U}=\mathcal{V}_{0}, \mathcal{V}=\mathcal{V}_{n}, y=X_{0}$ and $X=X_{n}$ we have, for $k=1, \ldots, n$ a cartesian square

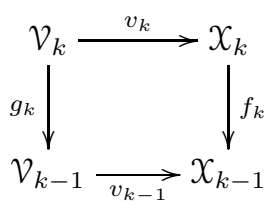

It is easy to check that each $v_{k}, k=0, \ldots, n$ satisfies the same hypotheses that $u$ satisfies, viz. $v_{k}$ is étale, adic and $\widehat{\mathcal{O}}_{\mathcal{V}_{k}, p} \rightarrow \widehat{\mathcal{O}}_{x_{k}, v_{k}(p)}$ is an isomorphism for every $p \in \mathcal{V}_{k}$. Since the proposition is true with $f=f_{k}$, therefore the diagram

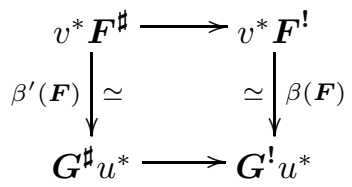

commutes, where the two horizontal arrows are $v^{*} \gamma^{!}(\boldsymbol{F})$ and $\gamma^{!}(\boldsymbol{G}) u^{*}$, the map $\beta^{\prime}(\boldsymbol{F}): v^{*} \boldsymbol{F}^{\sharp}=v^{\sharp} \boldsymbol{F}^{\sharp} \stackrel{\sim}{\longrightarrow} \boldsymbol{G}^{\sharp} u^{\sharp}=\boldsymbol{G}^{\sharp} u^{*}$ is the isomorphism that arises from the pseudofunctoriality of $\boldsymbol{-}^{\sharp}$ (indeed $v_{k}^{\sharp} f_{k}^{\sharp} \stackrel{\sim}{\longrightarrow} g_{k}^{\sharp} v_{k-1} \sharp$ ) and $\beta(\boldsymbol{F}): v^{*} \boldsymbol{F}^{!} \stackrel{\sim}{\longrightarrow} \boldsymbol{G}^{!} u^{*}$ is the natural isomorphism arising from the various base change isomorphisms $v_{k}^{*} f_{k}^{!} \stackrel{\sim}{\longrightarrow} g_{k}^{!} v_{k-1}^{*}$ (the obvious one if $f_{k}$ is an open immersion, and the map $\beta$ in AJL2 Theorem 7.4] when $f_{k}$ is pseudo-proper). Consider the diagram (with the inner square being (4.2.2.1) and the slanted arrows being the natural isomorphisms 
arising from the pseudofunctorial nature of $-\sharp$ and $-!$ )

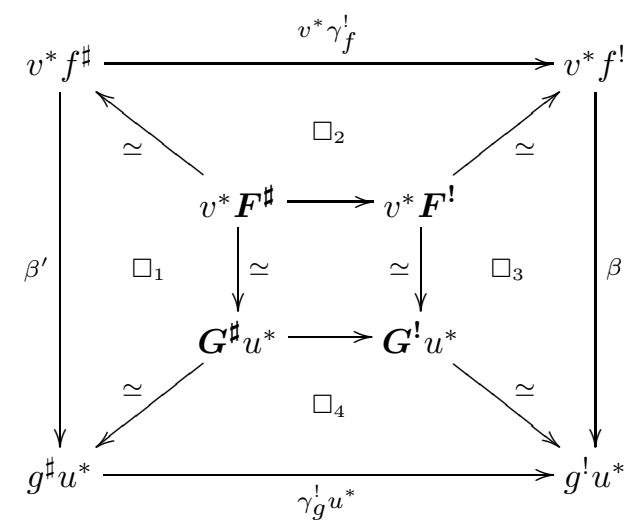

If we show that each of the subdiagrams $\square_{1}, \ldots, \square_{4}$ commutes, then, as the inner square (i.e. 4.2.2.1) ) commutes, the outer square will commute and the proposition will follow.

The subdiagram $\square_{1}$ commutes by the pseudo functoriality of $\square^{\sharp}$ (recall that $u^{*} \mid \operatorname{Coz}(y)=u^{\sharp}$ and $\left.v^{*} \mid \operatorname{Coz}(X)=v^{\sharp}\right)$. The subdiagrams $\square_{2}$ and $\square_{4}$ commute by the definitions of $\gamma_{f}^{!}$and $\gamma_{g}^{!}$. Subdiagram $\square_{3}$ commutes by (3.2.2).

It remains to prove the proposition when $f$ is pseudo-proper. By the universal property of $\left(g^{!}, \tau_{g}\right)$ it is enough to show

$$
\tau_{g}\left(u^{*} \mathcal{F}\right) \circ \mathbf{R} g_{*}\left(\gamma_{g}^{!}\left(u^{\sharp} \mathcal{F}\right) \circ \beta_{\mathcal{F}}^{\prime}\right)=\tau_{g}\left(u^{*} \mathcal{F}\right) \circ \mathbf{R} g_{*}\left(\beta_{\mathcal{F}} \circ v^{*} \gamma_{f}^{!}(\mathcal{F})\right) .
$$

Part of the proof (commutativity of (4.2.2.3) below) rests on the fact that at the punctual level the punctual trace $\operatorname{Tr}_{g, x^{\prime}}$ at a point $x^{\prime} \in \mathcal{V}$ equals the punctual trace $\operatorname{Tr}_{f, v\left(x^{\prime}\right)}$. We elaborate below.

Let $x \in \mathcal{X}$ and $\mathcal{G}=i_{x} f^{\sharp} \mathcal{F}(x)$. We will examine the map $\theta_{\mathcal{G}}$ under the identifications $\mathbf{R} g_{*} v^{*} \mathcal{G}=g_{*} v^{*} \mathcal{G}$ and $u^{*} \mathbf{R} f_{*} \mathcal{G}=u^{*} f_{*} \mathcal{G}$ (note that $v^{*} \mathcal{G}=v^{\sharp} \mathcal{G}$ is also flasque). It can be checked that $\theta_{\mathcal{G}}: u^{*} f_{*} \mathcal{G} \rightarrow g_{*} v^{*} \mathcal{G}$ is adjoint to $f_{*} \mathcal{G} \rightarrow f_{*} v_{*} v^{*} \mathcal{G}=u_{*} g_{*} v^{*} \mathcal{G}$.

Let $y=f(x), A=\widehat{\mathcal{O}}_{y, y}, R=\widehat{\mathcal{O}}_{x, x}, M=\mathcal{F}(y)$ and $\varphi: A \rightarrow R$ the map induced by $f$. Let $-\#$ be the pseudofunctor on zero-dimensional modules over local rings constructed by Huang in Hu1. Then $\left(f^{\sharp} \mathcal{F}\right)(x)=\varphi_{\#} M$. If $v^{-1}(x)$ is empty (so that $u^{-1}(y)$ is also empty) then $u^{*} f_{*} \mathcal{G}$ and $g_{*} v^{*} \mathcal{G}$ are both zero and $\theta_{\mathcal{G}}$ is the obvious map. The interesting case is when we have $p \in \mathcal{V}$ such that $x=v(p)$. Let $q=g(p)$. Using our hypothesis on $u: \mathfrak{U} \rightarrow \boldsymbol{y}$ we make the identifications $\widehat{\mathcal{O}}_{\mathcal{u}, q}=A$ and $\widehat{\mathcal{O}}_{\mathcal{V}, p}=R$ (since the isomorphisms underlying these are canonical). With these identifications, the map $\widehat{\mathcal{O}}_{\mathcal{u}, q} \rightarrow \widehat{\mathcal{O}}_{\mathcal{V}, p}$ induced by $g$ is again $\varphi: A \rightarrow R$.

Suppose $v^{-1}(x)=\left\{p_{1}, \ldots, p_{n}\right\}$. Set $q_{i}=g\left(p_{i}\right)$ for $i=1, \ldots, n$. Then $u^{-1}(y)=$ $\left\{q_{1}, \ldots, q_{n}\right\}$. We have $f_{*} i_{x} \varphi_{\#} M=i_{y} \varphi_{\#} M, g_{*} i_{p_{k}} \varphi_{\#} M=i_{q_{k}} \varphi_{\#} M, v_{*} i_{p_{k}} \varphi_{\#} M=$ $i_{x} \varphi_{\#} M, u_{*} i_{q_{k}} \varphi_{\#} M=i_{y} \varphi_{\#} M, v^{*} i_{x} \varphi_{\#} M=\oplus_{k} i_{p_{k}} \varphi_{\#} M$ and finally $u^{*} i_{y} \varphi_{\#} M=$ $\oplus_{k} i_{q_{k}} \varphi_{\#} M$. Thus in every case (whether $v^{-1}(x)$ is empty or not) we have

$$
v^{*} f_{*} \mathcal{G}=g_{*} u^{*} \mathcal{G}
$$

It is now easy to see that $\theta_{\mathcal{G}}: v^{*} f_{*} \mathcal{G} \rightarrow g_{*} u^{*} \mathcal{G}$ is the identity map. Indeed, the only interesting case is when $v^{-1}(x)$ is non-empty, and in this case, with previous notations, the map

$$
f_{*} \mathcal{G} \rightarrow f_{*} v_{*} v^{*} \mathcal{G}=u_{*} g_{*} v^{*} \mathcal{G}
$$


is the diagonal map $i_{y} \varphi_{\#} M \rightarrow \oplus_{k} i_{y} \varphi_{\#} M$. This proves the assertion on $\theta_{\mathcal{G}}$.

Now suppose $p, x, q, y$ satisfy $x=v(p), y=f(x)$ and $q=g(p)$. The $R$ modules $\left(f^{\sharp} \mathcal{F}\right)(x),\left(v^{\sharp} f^{\sharp} \mathcal{F}\right)(p)$ and $\left(g^{\sharp} u^{\sharp}\right) \mathcal{F}(p)$ are all equal to $\varphi_{\#} M$ and the natural isomorphism $\beta_{\mathcal{F}}^{\prime}(p):\left(v^{\sharp} f^{\sharp} \mathcal{F}\right)(p) \stackrel{\sim}{\longrightarrow}\left(g^{\sharp} u^{\sharp} \mathcal{F}\right)(p)$ is the identity map on $\varphi_{\#} M$. If $x$ is closed in the fiber $f^{-1}(y)$ (equivalently $p$ is closed in the fiber $g^{-1}(q)$ ) then, from the definitions of $\operatorname{Tr}_{f, x}$ and $\operatorname{Tr}_{g, p}$ we have

$$
\operatorname{Tr}_{f, x}(\mathcal{F})=\operatorname{Tr}_{g, p}\left(u^{\sharp} \mathcal{F}\right)=\operatorname{Tr}_{R / A, M}: \varphi_{\#} M \rightarrow M .
$$

The above assertions imply that the diagram

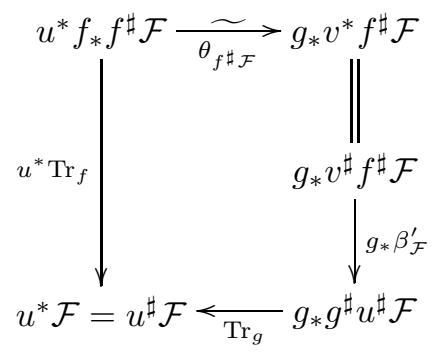

commutes.

Consider the following diagram:

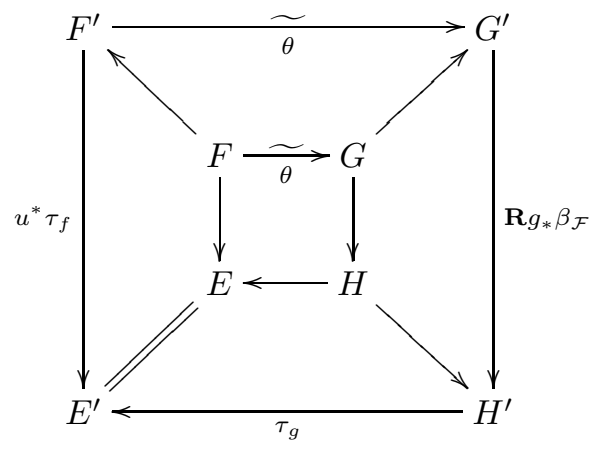

where

$$
\begin{aligned}
E & =u^{*} \mathcal{F}=u^{\sharp} \mathcal{F} \\
F & =u^{*} f_{*} f^{\sharp} \mathcal{F}=u^{*} \mathbf{R} f_{*} f^{\sharp} \mathcal{F} \\
G & =g_{*} v^{*} f^{\sharp} \mathcal{F}=g_{*} v^{\sharp} f^{\sharp} \mathcal{F}=\mathbf{R} g_{*} v^{*} f^{\sharp} \mathcal{F} \\
H & =g_{*} g^{\sharp} u^{\sharp} \mathcal{F}=\mathbf{R} g_{*} g^{\sharp} u^{\sharp} \mathcal{F} \\
E^{\prime} & =u^{*} \mathcal{F}=u^{\sharp} \mathcal{F}=E \\
F^{\prime} & =u^{*} \mathbf{R} f_{*} f^{!} \mathcal{F} \\
G^{\prime} & =\mathbf{R} g_{*} v^{*} f^{!} \mathcal{F} \\
H^{\prime} & =\mathbf{R} g_{*} g^{!} u^{\sharp} \mathcal{F}=\mathbf{R} g_{*} g^{!} u^{*} \mathcal{F} .
\end{aligned}
$$

The inner square is 4.2.2.3). The arrows pointing northeast and northwest arise from $\gamma_{f}^{!}\left(f^{\sharp} \mathcal{F}\right)$ while the southeast pointing arrow is from $\gamma_{g}^{!}\left(u^{\sharp} \mathcal{F}\right)$. The inner square, being (4.2.2.3), commutes. The outer square commutes by the definition of $\beta_{\mathcal{F}}$ (see AJL2 Definition 7.3]). Now consider the four trapezoids squeezed between the two 
squares. The top-most trapezoid commutes by the functoriality of $\theta$. The one's on the left and the bottom commute by the definition of $\gamma_{f}^{!}$and $\gamma_{g}^{!}$. This does not allow us (a priori) to conclude that the trapezoid on the right commutes - and we are not interested in showing this - but it does allow us to conclude that (4.2.2.2 holds ( since the arrows labeled $\theta$ can be reversed), which is what we wished to show. For completeness we point out (after the fact) that hence the remaining trapezoid also commutes.

REMARK 4.2.3. We have not stated Proposition 4.2.2 in its full generality; only as much as we need for the main conclusions of this paper. The assumption that the residue field of a point $q \in \mathcal{V}$ coincides with the residue field at $u(q)$ can be relaxed, but this would involve proving a base change for the punctual trace $\operatorname{Tr}_{f, x}$ for $x \in \mathcal{X}$ closed in $f^{-1}(f(x))$ and we lead us away from what we have set out to prove. One can also relax the assumption that $u$ is adic. In that case the conclusion would be that $\Gamma_{\mathcal{V}}^{\prime}\left(v^{*} \gamma_{f}^{!}(\mathcal{F})\right)$ is equivalent to $\gamma_{g}^{!}\left(u^{\sharp} \mathcal{F}\right)$. In greater detail, if the only assumption on $u$ is that it is étale, and $\beta_{\mathcal{F}}: \mathbf{R} \Gamma_{\mathcal{V}}^{\prime} v^{*} f^{!} \mathcal{F} \rightarrow g^{!} \mathbf{R} \Gamma_{\mathfrak{U}}^{\prime} u^{*} \mathcal{F}$ is the base change map in AJL2, Theorem 7.4], then, making the identifications $v^{\sharp} \mathcal{G}=\mathbf{R} \Gamma_{\mathcal{V}}^{\prime} v^{*} \mathcal{G}(\mathcal{G} \in \operatorname{Coz}(\mathcal{X}))$ and $u^{\sharp} \mathcal{F}=\mathbf{R} \Gamma_{\mathcal{u}}^{\prime} u^{*} \mathcal{F}$, one can prove that

$$
\beta_{\mathcal{F}} \circ \mathbf{R} \Gamma_{\mathcal{V}}^{\prime} v^{*} \gamma_{f}^{!}(\mathcal{F})=\gamma_{g}^{!}\left(u^{\sharp} \mathcal{F}\right) \circ \beta_{\mathcal{F}}^{\prime} .
$$

We leave the details (modulo the assertion on the behavior of the punctual trace) to the reader. We will have no occasion to use the more general assertion made in this remark.

\section{Smooth maps}

In this section we prove that $\gamma_{f}^{!}: Q_{x} f^{\sharp} \rightarrow f^{!} Q_{y}$ is an isomorphism when $f: x \rightarrow y$ is a smooth pseudo-finite type map. The proof we give can be broken into two parts: we show if there is any functorial isomorphism $\zeta_{f}: Q_{x} f^{\sharp} \stackrel{\sim}{\longrightarrow} f^{!} Q_{y}$ (one for each smooth $f$ ) then $\gamma_{f}^{!}$is also an isomorphism (this is essentially done in subsection (5.2); and we also show (essentially in Theorem 5.1.2) that there is indeed such an isomorphism $\zeta_{f}$. Indeed, in this paper, that is the only role played by Theorem 5.1.2 it provides a $\zeta_{f}$ as the inverse of $\xi_{f}$ (see (5.1.3.2)). We do not, in this paper, examine the relationship between the $\zeta_{f}$ we produce (through Verdier's classic trick) and $\gamma_{f}^{!}$(are they equal?). The principal difficulty in investigating this is our lack of knowledge of the composite

$$
\mathbf{R} f_{*} \mathbf{R} \Gamma_{x}^{\prime} \omega_{f}[d] \stackrel{\sim}{\longrightarrow} f_{*} f^{!} \mathcal{F} \stackrel{\tau_{f}}{\longrightarrow} \mathcal{O} y
$$

- the isomorphism coming from Theorem [5.1.2 for $f$ pseudo-proper smooth of relative dimension $d$. Here and below $\omega_{f}$ is as in [LNS Definition 2.6.4].

5.1. Verdier's isomorphism. We will be modifying Verdier's argument in V Theorem 3,p.397] to formal schemes to connect differential forms to duality. We begin with the formal scheme version of the fundamental local isomorphism Hrt p.180, Corollary III.7.3].

LEMMA 5.1.1. Let $f: x \rightarrow y$ be a closed immersion of noetherian formal schemes, and $\mathcal{J} \subset \mathcal{O}_{y}$ the coherent ideal which is the kernel of the natural surjective map $\mathcal{O}_{x} \rightarrow f_{*} \mathcal{O}_{y}$. Suppose $\mathcal{J}$ is locally generated by a regular sequence of d elements 
so that $\mathcal{J} / \mathcal{J}^{2}$ can be regarded as a locally free $\mathcal{O}_{x}$-module of rank $d$. Then for $\mathcal{F} \in \widetilde{\mathbf{D}}_{\mathrm{qc}}^{+}(\mathrm{y})$ we have a functorial isomorphism

$$
f^{!} \mathcal{F} \simeq \Gamma_{x}^{\prime}\left(\mathbf{L} f^{*} \mathcal{F}\right) \otimes x \wedge_{\mathcal{O}_{x}}^{d}\left(\mathcal{J} / \mathcal{J}^{2}\right)^{*}[-d] .
$$

Proof. The natural map $f^{!} \mathbf{R} \Gamma_{y}^{\prime} \rightarrow f^{!}$is an isomorphism by AJL2 Corollary6.1.5(b)]. Using this and AJL2 Example6.1.3(4)] we conclude that for $\mathcal{F} \in \widetilde{\mathbf{D}}_{\mathrm{qc}}^{+}(\mathrm{y})$, we have a functorial isomorphism

$$
f^{!} \mathcal{F} \stackrel{\sim}{\longrightarrow} \bar{f}^{*} \mathbf{R} \mathcal{H o m} \dot{y}\left(f_{*} \mathcal{O}_{x}, \mathbf{R} \Gamma_{y}^{\prime} \mathcal{F}\right) .
$$

The rest of the proof is as as in [Hrt p.180, Corollary III.7.3] using "way-out". Note that since $f$ is adic we have $\mathbf{L} f^{*} \mathbf{R} \Gamma_{y}^{\prime}=\mathbf{R} \Gamma_{x}^{\prime} \mathbf{L} f^{*}$ (cf. [AJL2, Corollary 5.2.11(c)]).

For the next result, recall that $\mathbb{G}$ is the category whose objects are noetherian formal schemes and whose morphisms are composites of pseudo-proper maps and open immersions.

TheOREm 5.1.2. Suppose a morphism $f: X \rightarrow y$ in $\mathbb{G}$ is smooth of relative dimension d. For $\mathcal{F} \in \widetilde{\mathbf{D}}_{\mathrm{qc}}^{+}(\mathrm{y})$ we have a functorial isomorphism

$$
\mathbf{R} \Gamma_{x}^{\prime}\left(f^{*} \mathcal{F} \otimes x \omega_{f}[d]\right) \stackrel{\sim}{\longrightarrow} f^{!} \mathcal{F} .
$$

Proof. Let $X^{2}=X \times y X, p_{1}, p_{2}: X^{2} \rightarrow X$ the two projections and $\delta: X \hookrightarrow X^{2}$ the diagonal (cf. commutative diagram below).

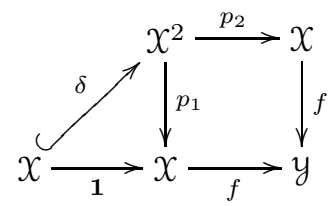

Note that $\delta$ is adic and is a closed immersion. If $\partial_{\delta} \subset \mathcal{O}_{x^{2}}$ is the ideal of the closed immersion $\delta$ then $\partial_{\delta}$ is locally given by a regular sequence, and one checks, as follows, that $\wedge_{\mathcal{O} x}^{d}\left(\mathcal{J}_{\delta} / \mathcal{J}_{\delta}^{2}\right)=\omega_{f}$. First, by [LNS Prop. 2.6.8] applied to the composite

$$
X \stackrel{\delta}{\rightarrow} X^{2} \stackrel{p_{1}}{\longrightarrow} X
$$

we see that $\delta^{*} \widehat{\Omega}_{p_{1}}^{1}=\mathcal{J}_{\delta} / \mathcal{J}_{\delta}^{2}$. Next, by applying [LNS Prop. 2.6.6] to the product $\left(X^{2}, p_{1}, p_{2}\right)$, we see that $\widehat{\Omega}_{X / y}^{1}$ can be identified with $\mathcal{J}_{\delta} / \mathcal{J}_{\delta}^{2}$, whence $\omega_{f}=$ $\wedge_{\mathcal{O} X}^{d}\left(\mathcal{J}_{\delta} / \mathcal{J}_{\delta}^{2}\right)$. The above results on differentials are probably true in greater generality (as they are for ordinary schemes), and can presumably be proven via the techniques in [EGA-IV] p. 126].

Consider the commutative diagram above (with $\mathbf{1}=\mathbf{1}_{x}$, the identity map). According to [AJL2, Theorem 3] (see also [AJL2, Theorem 7.4]) we have

$$
p_{1}^{!} f^{*} \mathcal{F} \stackrel{\sim}{\longrightarrow} \Gamma_{\chi^{2}}^{\prime} p_{2}^{*} f^{!} \mathcal{F} \text {. }
$$

Since $f$ is not assumed to be pseudo-proper, we have implicitly used the localization theorems for $-!$ proved by S. Nayak $\mathbf{N a y}$ (See also (3.2.1)). Applying $\delta$ ! to both sides of the above isomorphism we get, using $\delta^{!} p_{1}^{!} \simeq \mathbf{1}^{!}=\mathbf{R} \Gamma_{\chi}^{\prime}$,

$$
\begin{aligned}
\mathbf{R} \Gamma_{X}^{\prime} f^{*} \mathcal{F} & \stackrel{\sim}{\longrightarrow} \delta^{!} \mathbf{R} \Gamma_{X^{2}}^{\prime} p_{2}^{*} f^{!} \mathcal{F} \\
& \left.\stackrel{\sim}{\longrightarrow}\left(\mathbf{R} \Gamma_{X}^{\prime} \mathbf{L} \delta^{*} p_{2}^{*} f^{!} \mathcal{F}\right) \otimes x \omega_{f}^{*}[-d]\right)
\end{aligned}
$$


(the second isomorphism is via the Lemma). This means we have

$$
\begin{aligned}
\mathbf{R} \Gamma_{x}^{\prime}\left(f^{*} \mathcal{F} \otimes \omega_{f}[d]\right) & \stackrel{\sim}{\longrightarrow} \mathbf{R} \Gamma_{X}^{\prime} \mathbf{L} \delta^{*} p_{2}^{*} f^{!} \mathcal{F} \\
& \stackrel{\sim}{\longrightarrow} \Gamma_{x}^{\prime} f^{!} \mathcal{F}=f^{!} \mathcal{F} \quad\left(\text { since } f^{!} \mathcal{F} \in \mathbf{D}_{\mathrm{qct}}(X)\right) .
\end{aligned}
$$

Remark 5.1.3. Let $\left(X, \Delta^{\prime}\right) \stackrel{f}{\rightarrow}(\mathcal{Y}, \Delta)$ be a smooth map in $\mathbb{F}_{c}^{*}$. Assume that the relative dimension is constant, and is $d$. By [LNS Lemma 5.1.3] and [LNS Main Theorem (iii)] for $\mathcal{F} \in \mathrm{Coz}_{\Delta}(\mathrm{y})$ we have an isomorphism

$$
\Gamma_{x}^{\prime}\left(f^{*} \mathcal{F} \otimes x \omega_{f}[d)\right] \stackrel{\sim}{\longrightarrow} Q_{x} f^{\sharp} \mathcal{F}
$$

and hence a functorial isomorphism

$$
\xi_{f}(\mathcal{F}): f^{!} \mathcal{F} \stackrel{\sim}{\longrightarrow} Q x f^{\sharp} \mathcal{F}
$$

given by $\xi_{f}=(5.1 .3 .1) \circ(5.1 .2 .1)^{-1}$. This gives a unique map (functorial in $\mathcal{F} \in$ $\left.\mathrm{Coz}_{\Delta}(y)\right)$ in $\mathrm{Coz}_{\Delta^{\prime}}(X)$

$$
\lambda_{f}(\mathcal{F}): f^{\sharp} \mathcal{F} \longrightarrow f^{\sharp} \mathcal{F}
$$

such that $Q_{x}\left(\lambda_{f}\right)=\xi_{f} \circ \gamma_{f}^{!}$. It is elementary to see that $\gamma_{f}^{!}(\mathcal{F})$ is an isomorphism if and only if $\lambda_{f}(\mathcal{F})$ is an isomorphism. Note that $\lambda_{f}$ is defined even when the relative dimension is not constant (by defining it separately on each connected component), and in this case also $\lambda_{f}(\mathcal{F})$ is an isomorphism if and only if $\gamma_{f}^{!}(\mathcal{F})$ is. In this paper we do not attempt to nail down $\lambda_{f}$. This question is, at the bottom, the same as the question of the relationship between $\xi_{f}$ and $\gamma_{f}^{\prime}$ discussed briefly at the beginning of this section.

5.2. Smooth pseudo-finite maps. Suppose $f:\left(X, \Delta^{\prime}\right) \rightarrow(y, \Delta)$ is a map in $\mathbb{F}_{c}^{*}$ which is smooth and pseudo-finite. In this situation we note that $f_{*} \mathcal{F}$ is in $\operatorname{Coz}(y)$ for every $\mathcal{F} \in \operatorname{Coz}(X)$. Moreover, in addition to the trace map $\operatorname{Tr}_{f}$ : $f_{*} f^{\sharp} \rightarrow 1$ between functors on $\operatorname{Coz}(y)$ we have another trace map (arising from Grothendieck duality)

$$
\operatorname{Tr}_{f}^{*}: f_{*} f^{\sharp} \rightarrow \mathbf{1}
$$

defined as follows. We have the map

$$
Q y\left(f_{*} f^{\sharp} \mathcal{F}\right)=\mathbf{R} f_{*} Q x\left(f^{\sharp} \mathcal{F}\right) \stackrel{\xi_{f}^{-1}}{\longrightarrow} \mathbf{R} f_{*} f^{!} \mathcal{F} \stackrel{\tau_{f}(\mathcal{F})}{\longrightarrow} \mathcal{F}=Q_{y}(\mathcal{F}) .
$$

Since $f_{*} f^{\sharp} \mathcal{F}$ and $\mathcal{F}$ lie in $\operatorname{Coz}_{\Delta}(y)$, we define $\operatorname{Tr}_{f}^{*}(\mathcal{F})$ as the unique map of Cousin complexes satisfying $\tau_{f}(\mathcal{F})=Q_{y}\left(\operatorname{Tr}_{f}^{*}(\mathcal{F})\right.$ ). It is easy to check that $\operatorname{Tr}_{f}^{*}$ is functorial in $\mathcal{F} \in \operatorname{Coz}(y)$. The two traces are clearly related via the equation

$$
\operatorname{Tr}_{f}^{*} \circ f_{*} \lambda_{f}=\operatorname{Tr}_{f} \text {. }
$$

This new trace $\operatorname{Tr}_{f}^{*}$ has the expected universal property, viz.:

Lemma 5.2.2. For $f$ as above and $\mathcal{F}$ a Cousin complex on $(y, \Delta)$ the pair $\left(f^{\sharp} \mathcal{F}, \operatorname{Tr}_{f}^{*}(\mathcal{F})\right)$ represents the functor $F(\mathcal{G})=\operatorname{Hom}_{\Delta}\left(f_{*} \mathcal{G}, \mathcal{F}\right)$ of Cousin complexes $\mathcal{G}$ on $\left(X, \Delta^{\prime}\right)$.

Proof. Suppose we have a map of complexes $\varphi: f_{*} \mathcal{G} \rightarrow \mathcal{F}$. By the universal property of $\left(f^{!}, \tau_{f}\right)$ we get a unique map in $\varphi^{\prime}: \mathcal{G} \rightarrow f^{\sharp}(\mathcal{F})$ in $\mathbf{D}_{\mathrm{qc}}^{+}(X)$ such that $\operatorname{Tr}_{f}^{*} \mathbf{R} f_{*} \varphi^{\prime}=\varphi$. Since $\mathcal{G}$ and $f^{\sharp} \mathcal{F}$ are in $\operatorname{Coz}(X)$ therefore $\varphi^{\prime}$ has a unique representative (which we also denote $\varphi^{\prime}$ ) in $\operatorname{Coz}(X)$. Since $f_{*} \mathcal{G}, f_{*} f^{\sharp} \mathcal{F}$ and $\mathcal{F}$ are all 
in $\mathrm{Coz}_{\Delta}(\mathrm{y})$, by Suominen's results in $\mathbf{S u}$, the relationship between $\mathbf{R} f_{*} \varphi^{\prime}$ and $\varphi$ translates to an equality (in $\operatorname{Coz}(y)) \operatorname{Tr}_{f}^{*} f_{*} \varphi^{\prime}=\varphi$. This proves the Lemma.

EXAMPLES 5.2.3. We give a few examples of $f$ smooth and pseudo-finite for which $\lambda_{f}$ is an isomorphism. These are more or less an immediate consequence of Lemma 5.2.2 The idea is to show that $\left(f^{\sharp} \mathcal{F}, \operatorname{Tr}_{f}(\mathcal{F})\right)$ represents the functor $F$ in the Lemma. Note that if we show this for $f$ and $\mathcal{F}$ as above we would indeed have proved (via (5.2.1)) that $\lambda_{f}(\mathcal{F})$ (and hence $\gamma_{f}^{!}(\mathcal{F})$ ) is an isomorphism.

(1) Let $(\mathcal{X}, \Delta) \in \mathbb{F}_{c}$ with $\mathcal{J} \in \mathcal{O}_{X}$ a coherent ideal containing an ideal of definition of $X$. Denote by $X^{*}$ the completion of $X$ with respect to $\mathcal{J}$ and by $\kappa$ : $X^{*} \rightarrow X$ the resulting map. The map $\kappa$ is pseudo-finite and smooth of relative dimension zero (i.e. $\kappa$ is étale and pseudo-proper). For $\mathcal{F} \in \operatorname{Coz}(X)$ and $\mathcal{G} \in$ $\operatorname{Coz}\left(X^{*}\right)$ one checks via LNS Lemma 10.3.8(i)], [Ibid., Prop. 10.3.9] and especially [Ibid., Remark, 10.3.10] that $\mathbf{R} \Gamma_{\mathfrak{\jmath}} \mathcal{F}=\Gamma_{\mathfrak{J}} \mathcal{F}, \kappa^{\sharp} \mathcal{F}=\kappa^{*} \Gamma_{\mathcal{\jmath}} \mathcal{F}, \kappa_{*} \kappa^{\sharp} \mathcal{F}=\Gamma_{\mathfrak{\jmath}} \mathcal{F}$ and $\kappa^{*} \kappa_{*} \mathcal{G}=\mathcal{G}$ (see [AJL2 Proposition 5.2.8] for the last relation). Needless to say, we are using equality signs for many well-known canonical isomorphisms.

The Trace map $\operatorname{Tr}_{\kappa}(\mathcal{F}): \kappa_{*} \kappa^{\sharp} \mathcal{F} \rightarrow \mathcal{F}$ is the natural map $\Gamma_{\mathcal{f}} \mathcal{F} \hookrightarrow \mathcal{F}$. Let $F$ be the functor

$$
F=\operatorname{Hom}_{\Delta}\left(\kappa_{*}-, \mathcal{F}\right)
$$

on $\operatorname{Coz}_{\kappa^{\sharp} \Delta}\left(X^{*}\right)$. If $\mathcal{G} \in \operatorname{Coz}\left(X^{*}\right)$, then $\Gamma_{\mathcal{J}} \kappa_{*} \mathcal{G}=\kappa_{*} \mathcal{G}$ and any map $\kappa_{*} \mathcal{G} \stackrel{\psi}{\rightarrow} \mathcal{F}$ in $\operatorname{Coz}(X)$ must factor (uniquely) as

$$
\kappa_{*} \mathcal{G} \stackrel{\psi^{\prime}}{\longrightarrow} \Gamma_{\mathfrak{J}} \mathcal{F} \stackrel{\operatorname{Tr}_{\kappa}}{\longrightarrow} \mathcal{F} .
$$

Now $\kappa^{*}\left(\psi^{\prime}\right)$ is a map from $\mathcal{G}$ to $\kappa^{*} \Gamma_{\mathfrak{J}} F=\kappa^{\sharp} \mathcal{F}$ and $\kappa_{*} \kappa^{*}\left(\psi^{\prime}\right)=\psi^{\prime}$. Thus $\varphi=\kappa^{*}\left(\psi^{\prime}\right)$ solves $\operatorname{Tr}_{\kappa}(\mathcal{F}) \kappa_{*} \varphi=\psi$, and this is the only solution. Indeed for any solution $\varphi$ we have $\kappa_{*} \varphi=\psi^{\prime}$ by the uniqueness of the factorization (5.2.3.1) above, and hence $\varphi=\kappa^{*} \kappa_{*}(\varphi)=\kappa^{*}\left(\psi^{\prime}\right)$. We have therefore proven that $\left(\kappa^{\sharp} \mathcal{F}, \operatorname{Tr}_{\kappa}(\mathcal{F})\right)$ represents $F$ and hence that $\lambda_{\kappa}$ and $\gamma_{\kappa}^{!}$are isomorphisms.

If $\mathcal{X}=\operatorname{Spf}(R), J \subset R$ the open ideal corresponding to $\mathcal{J}, F=\Gamma(\mathcal{X}, \mathcal{F})$, $R^{*}$ the $J$-adic completion of $R$, then $\Gamma\left(X^{*}, \kappa^{\sharp} \mathcal{F}\right)=\Gamma_{J} F, \kappa^{\sharp} \mathcal{F}=\Gamma_{J} F^{\sim R^{*}}$ and $\kappa_{*} \kappa^{\sharp} \mathcal{F}=\Gamma_{J} F^{\sim R}$. The map $\operatorname{Tr}_{\kappa}$ corresponds to the inclusion $\Gamma_{J} F \subset F$.

(2) Let $(R, \mathfrak{m}, k)$ be a complete noetherian local ring, $X=\operatorname{Spf}(R, \mathfrak{m})$ and $x \in X$ the unique point in $X$. Suppose that $(\mathcal{Y}, \Delta)$ is an object in $\mathbb{F}_{c}$ and that $f: X \rightarrow Y$ a smooth pseudo-proper map of relative dimension $d$. Note that $f$ is pseudo-finite. Let $y=f(x)$ and $\Delta(y)=p$ so that $f^{\sharp} \Delta(x)=p$. Note that $y$ is a closed point of $y$. Set $A=\mathcal{O}_{y, y}$ and $\omega_{R}=\Gamma\left(X, \omega_{f}\right)$. For $\mathcal{F} \in \mathrm{Coz}_{\Delta}(y)$ we may-via 2.3.2 -make the identification

$$
f^{\sharp} \mathcal{F}=i_{x}\left(\mathrm{H}_{\mathfrak{m}}^{d}\left(\mathcal{F}(y) \otimes \omega_{R}\right)\right)[-p] .
$$

If

$$
\operatorname{res}_{R / A}: \mathrm{H}_{\mathfrak{m}}^{d}\left(\mathcal{F}(y) \otimes \omega_{R}\right) \rightarrow \mathcal{F}(y)
$$

is the residue map (2.3.3) then the trace map $\operatorname{Tr}_{f}(\mathcal{F}): f_{*} f^{\sharp} \mathcal{F} \rightarrow \mathcal{F}$ is the composite

$$
i_{y}\left(\mathrm{H}_{\mathfrak{m}}^{d}\left(\mathcal{F}(y) \otimes \omega_{R}\right)\right)[-p] \stackrel{\operatorname{viares}_{R / A}}{\longrightarrow} i_{y} \mathcal{F}(y)[-p] \stackrel{\text { natural }}{\longrightarrow} \mathcal{F} .
$$

We want to show that $\left(f^{\sharp} \mathcal{F}, \operatorname{Tr}_{f}(\mathcal{F})\right)$ represents the functor $F$ in Lemma 5.2.2 Without loss of generality we assume that $p=0$. By (2.2.2) $\left(f^{\sharp} \mathcal{F}, i_{y} \operatorname{res}_{R / A}\right)$ 
represents the functor $\operatorname{Hom}_{\Delta}\left(f_{*}-, \mathcal{F}(y)^{s}\right)$ on $\mathrm{Coz}_{f^{\sharp}}(X)$. It follows easily that $\left(f^{\sharp} \mathcal{F}, \operatorname{Tr}_{f}(\mathcal{F})\right)$ represents the functor

$$
F=\operatorname{Hom}_{\Delta}\left(f_{*}-, \mathcal{F}\right)
$$

on $\operatorname{Coz}_{f} \Delta(X)$. Thus this is another instance where $\lambda_{f}$, and hence $\gamma_{f}^{!}$is an isomorphism.

The following lemma, based on the above examples, is very useful in establishing that $\gamma_{f}^{!}$is an isomorphism for smooth $f$.

Lemma 5.2.4. Let $(X, \Delta)$ be an object of $\mathbb{F}_{c}, x \in X$ a closed point, $R$ the completion of $\mathcal{O}_{x, x}$ at its maximal ideal, and

$$
\kappa: X^{*}:=\operatorname{Spf}\left(R, \mathfrak{m}_{R}\right) \longrightarrow X
$$

the resulting pseudo-finite étale map. For a map $\alpha: \mathcal{F} \rightarrow \mathcal{G}$ in $\mathrm{Coz}_{\Delta}(\mathcal{X})$ the following are equivalent

(a) $\kappa^{!}\left(Q_{x}(\alpha)\right)$ is an isomorphism;

(b) $\alpha(x): \mathcal{F}(x) \rightarrow \mathcal{G}(x)$ is an isomorphism.

Proof. Without loss of generality, we may assume that $\Delta(x)=0$. Note that $\kappa$ is a special case of (1) and (2) in 5.2 .3 and hence we have isomorphisms

$$
\kappa^{!} \circ Q_{x} \stackrel{\sim}{\longrightarrow} i_{x}[(\cdot)(x)]
$$

(and $\operatorname{Tr}_{\kappa}(\mathcal{F})$ may be identified with the inclusion $i_{x} \mathcal{F}(x) \hookrightarrow \mathcal{F}$ for $\mathcal{F} \in \operatorname{Coz}(X)$ ). The Lemma follows.

5.3. The isomorphism theorem for smooth maps. Let $(A, \mathfrak{m})$ be a local ring and assume $A$ is complete with respect to an ideal $I$ (not necessarily $\mathfrak{m}$-primary) of $A$. Let $y=\operatorname{Spf}(A, I), y \in y$ the unique closed point, $\Delta$ a codimension function on $\boldsymbol{y}$, and $p=\Delta(y)$. Assume $\boldsymbol{y} \in \mathbb{F}$. Let $K$ be an $A$-module which is an injective hull of the residue field of $A$, and consider the $\mathcal{O}_{y}$-module $K^{\sim A} \in \mathcal{A}_{\mathrm{qct}}(\mathrm{y})$. Set $\mathcal{F}=K^{\sim A}[-p]$. Note that $\left.\mathcal{F} \in \mathrm{Coz}_{(} \boldsymbol{y}\right)$.

LEMma 5.3.1. In the above situation, if $f:\left(X, \Delta^{\prime}\right) \rightarrow(\mathcal{Y}, \Delta)$ is a smooth map in $\mathbb{F}_{c}^{*}$, then $\lambda_{f}(\mathcal{F})$ is an isomorphism.

Proof. Without loss of generality we may assume that $p=\Delta(y)=0$. To reduce notational clutter we write $\mathcal{R}=f^{\sharp} \mathcal{F}$ and $\lambda=\lambda_{f}(\mathcal{F})$. Since the question is local on $\mathcal{X}$ we assume $\mathcal{X}=\operatorname{Spf}(R, J)$. Note that if $\mathcal{J}^{\prime}$ is the coherent $\mathcal{O}_{X}$ ideal corresponding to the open ideal $\mathfrak{m} R+J$ of $(R, J)$ then $\Gamma_{g^{\prime}}^{\prime} \mathcal{R}=\mathcal{R}$ and therefore

$$
\Gamma_{\mathcal{J}^{\prime}}^{\prime}(\lambda)=\lambda
$$

We first prove the Lemma under the assumption that $I$ is $\mathfrak{m}$-primary, i.e. $(A, \mathfrak{m})$ is a complete local ring and $y=\operatorname{Spf}(A, \mathfrak{m})$.

Now $\mathcal{F}$ is a residual complex on $y$ (cf. [LNS $\S \S 9.1]$ ). By [LNS Proposition 9.1.4] we conclude that $\mathcal{R}$ is a residual complex on $X$.

We have a natural isomorphism of $R$-modules (from the definition of $t$-dualizing complexes, and from the nature of maps between Cousin complexes)

$$
R \stackrel{\sim}{\longrightarrow} \operatorname{Hom}_{\Delta^{\prime}}(\mathcal{R}, \mathcal{R})=:[\mathcal{R}, \mathcal{R}]
$$

given by $r \mapsto \mu_{r}, \mu_{r}=$ multiplication by $r$. In particular $\lambda=\lambda_{f}(\mathcal{F})$ corresponds to a unique element $r_{f}$ in $R$. We have to show that $r_{f}$ is a unit in $R$ (this is equivalent to showing that $\lambda$ is an isomorphism). This is the same as showing that the image 
of $r_{f}$ in $\widehat{R}_{\mathfrak{n}}$ is a unit for every maximal ideal $\mathfrak{n}$ of $R\left(\widehat{R}_{\mathfrak{n}}=\right.$ completion of $R$ at $\mathfrak{n})$. Pick such an $\mathfrak{n}$; it corresponds to a closed point $x$ of $\mathcal{X}$. With $[\mathcal{R}(x), \mathcal{R}(x)]:=$ $\operatorname{Hom}_{R}(\mathcal{R}(x), \mathcal{R}(x))$, we have a commutative diagram

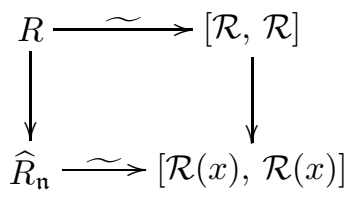

where the left column is the usual completion map and the right column is the result of applying the functor $(\cdot)(x)$ on morphisms in $\operatorname{Coz}(X)$. The horizontal arrow at the bottom is $\hat{r} \mapsto \mu_{\hat{r}}$, where $\mu_{\hat{r}}$ is multiplication by $\hat{r}$. (Note that $\mathcal{R}(x)$ is an $\widehat{R}_{\mathfrak{n}}$ module being the injective hull of the residue field of $R_{\mathfrak{n}}$.) This bottom arrow is an isomorphism by Matlis duality. Thus the image of $r_{f}$ in $\widehat{R}_{\mathfrak{n}}$ is a unit if and only if $\lambda(x): \mathcal{R}(x) \rightarrow \mathcal{R}(x)$ is an isomorphism.

Let $X^{*}=X_{\mathfrak{n}}^{*}=\operatorname{Spf}\left(\widehat{R}_{\mathfrak{n}}\right)$ (cf. Subsection 2.1) and $\kappa: X^{*} \rightarrow X$ the natural map. By Theorem 4.1.4(d) we have a commutative diagram

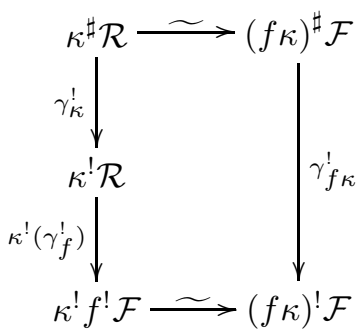

According to $5.2 .3(2), \gamma_{f \kappa}^{!}$and $\gamma_{\kappa}^{!}$are isomorphisms. Therefore $\kappa^{!}\left(\gamma_{f}^{!}\right)$-or, equivalently $\kappa^{!}\left(Q_{x} \lambda\right)$-is an isomorphism. By Lemma 5.2.4 this means $\lambda(x)$ is an isomorphism. Thus we are done if $y=\operatorname{Spf}(A, \mathfrak{m})$. (See also $[\mathbf{S}]$ p.124, Lemma 3]).

Suppose $I$ is not $\mathfrak{m}$-primary. Let $\widehat{A}$ be the $\mathfrak{m}$-adic completion of $A, S$ the $\mathfrak{m} R+J$ adic completion of $R$ and $\operatorname{set} \mathcal{U}:=\operatorname{Spf}(\widehat{A}, \mathfrak{m} \widehat{A}), \mathcal{V}:=\operatorname{Spf}(S, \mathfrak{m} S+J S)$. Let $u: \mathcal{U} \rightarrow \mathcal{y}$, $v: \mathcal{V} \rightarrow X, g: \mathcal{V} \rightarrow \mathcal{U}$ be the natural maps. Note that $\mathcal{V}=\mathcal{U} \times y \mathcal{X}$ and $v, g$ are the two projections. The two maps $u$ and $v$ both satisfy the hypothesis on the map $\kappa$ in 5.2.3 (1). Hence $\gamma_{u}^{!}$and $\gamma_{v}^{!}$are isomorphisms. Now $u^{\sharp} \mathcal{F}=K^{\sim \widehat{A}}$, and therefore (by what we proved for the case where $I$ is $\mathfrak{m}$-primary) $\gamma_{g}^{!}\left(u^{\sharp} \mathcal{F}\right)$ is an isomorphism. Using Theorem 4.1.4(d) we see that $\gamma_{u g}^{!}(\mathcal{F})$ is an isomorphism (since $\gamma_{u}^{!}$and $\gamma_{g}^{!}\left(u^{\sharp} \mathcal{F}\right)$ are isomorphisms). In other words $\gamma_{f v}^{!}(\mathcal{F})$ is an isomorphism. This coupled with the fact that $\gamma_{v}^{!}$is an isomorphism gives (via another application of loc.cit.) that $v^{!}\left(\gamma_{f}^{!}(\mathcal{F})\right)$ is an isomorphism. Since $Q_{x}(\lambda)=\xi_{f} \circ \gamma_{f}^{!}$(see (5.1.3.3) $)$, this implies that $v^{!} Q_{x}(\lambda)$ is an isomorphism. Now $\gamma_{v}^{!}$is an isomorphism-by 5.2.3](1) - and hence $Q_{v} v^{\sharp}(\lambda)$ is an isomorphism. In other words $v^{\sharp}(\lambda)$ is an isomorphism in $\operatorname{Coz}(\mathcal{V})$. Now, on $\operatorname{Coz}(X)$ we have the identity of functors $v_{*} v^{\sharp}=\Gamma_{\gamma^{\prime}}$. Therefore using [5.3.1.1) we get $\lambda=\Gamma_{\mathcal{J}^{\prime}}(\lambda)=v_{*} v^{\sharp}(\lambda)$ (the last via [LNS, 10.3.8(i), 10.3.9, 10.3.10]) proving that $\lambda$ is an isomorphism.

Theorem 5.3.2. Let $f:\left(\mathcal{X}, \Delta^{\prime}\right) \rightarrow(\mathcal{Y}, \Delta)$ be a smooth map in $\mathbb{F}_{c}^{*}$. Then the functorial maps $\gamma_{f}^{!}: Q_{x} f^{\sharp} \rightarrow f^{!} Q_{y}$ and $\lambda_{f}: f^{\sharp} \rightarrow f^{\sharp}$ are isomorphisms. 
Proof. It is enough to show that $\lambda_{f}$ is an isomorphism. The question is clearly local on $X$ and by Theorem 4.1.4 (c) it is local on $y$ too. So without loss of generality we assume that $\mathcal{X}=\operatorname{Spf}(R, J)$ and $\mathcal{Y}=\operatorname{Spf}(A, I)$ with $\mathcal{J}=J^{\sim R}$ and $\mathcal{J}=I^{\sim A}$ being the corresponding coherent ideal sheaves on $X$ and $y$ respectively.

We reduce to the case where the Krull dimension of $y$ is finite. to that end, let $y \in \mathcal{y}$ be a point, $A^{*}$ the $\mathcal{J}_{y}$-adic completion of $\mathcal{O}_{y, y}, \mathcal{U}=\operatorname{Spf}\left(A^{*}, I A^{*}\right)$ and $u: \mathcal{U} \rightarrow \mathcal{y}$ the resulting adic étale map. Consider the cartesian square (4.2.1). To emphasize the role of $y$, write $v_{y}=v$ and $g_{y}=g$. Now $\gamma_{f}^{!}$is an isomorphism if and only if $v_{y}^{*}\left(\gamma_{f}^{\prime}\right)$ is an isomorphism for every $y \in \mathcal{y}$. By Proposition 4.2.2 this is true if $\gamma_{g_{y}}^{!}$is an isomorphism for every $y \in \mathcal{Y}$. Since $\mathcal{U}$ has finite Krull dimension, the theorem is true if it is true for bases $y$ of finite Krull dimension and we restrict ourselves to this case for the rest of the proof.

Since $y$ is finite dimensional, all Cousin complexes on $y$ are bounded. For $\mathcal{F} \in \operatorname{Coz}(\mathrm{y})$ and $n \in \mathbb{Z}$ we let $\mathcal{F}^{n}$ denote the degree $n$ component of $\mathcal{F}$. For $\mathcal{F} \neq 0$ let $a(\mathcal{F})=\max \left\{n \mid \mathcal{F}^{n} \neq 0\right\}-\min \left\{n \mid \mathcal{F}^{n} \neq 0\right\}+1$. Note that for $\mathcal{F} \neq 0$, $a(\mathcal{F})<\infty$. The theorem is clearly true for $\mathcal{F}=0$. We prove the result for non-zero $\mathcal{F}$ by induction on $a(\mathcal{F})$. So suppose the theorem is true for $\mathcal{G} \in \operatorname{Coz}(\mathrm{y})$ such that $1 \leq a(\mathcal{G})<l$. Suppose $a(\mathcal{F})=l$. Let $p=\min \left\{n \mid \mathcal{F}^{n} \neq 0\right\}$. We have a short exact sequence of Cousin complexes on $(y, \Delta)$

$$
0 \longrightarrow \mathcal{G} \longrightarrow \mathcal{F} \longrightarrow \mathcal{F}^{p}[-p] \longrightarrow 0
$$

where $\mathcal{F} \rightarrow \mathcal{F}^{p}[-p]$ is the natural projection. By [NS Lemma 10.2.4]

$$
0 \longrightarrow f^{\sharp} \mathcal{G} \longrightarrow f^{\sharp} \mathcal{F} \longrightarrow f^{\sharp}\left(\mathcal{F}^{p}[-p]\right) \longrightarrow 0
$$

is exact. Now $a\left(\mathcal{F}^{p}[-p]\right)=1<l$ and $a(\mathcal{G}) \leq l-1$ and and hence by our induction hypothesis $\lambda_{f}(\mathcal{G})$ and $\lambda_{f}\left(\mathcal{F}^{p}[-p]\right)$ are isomorphisms. Since $\lambda_{f}$ is functorial, it follows that $\lambda_{f}(\mathcal{F})$ is an isomorphism.

It remains to prove the theorem when $a(\mathcal{F})=1$. In this case $\mathcal{F}=\mathcal{H}[-p]$ where $\mathcal{H}=\oplus_{\Delta(y)=p} i_{y} M_{y}$ where $M_{y}$ is a zero dimensional $\mathcal{O}_{y, y}-$ module. So, without loss of generality, we assume that $\mathcal{F}=i_{y} M_{y}[q]$ ( $M_{y}$ a zero dimensional $\mathcal{O}_{y, y}$-module) for some point $y \in \mathcal{y}$ with $\Delta(y)=-q$. We again set $\mathcal{U}=\operatorname{Spf}\left(A^{*}, I A^{*}\right)$ where $A^{*}$ is the completion of $\mathcal{O}_{y, y}$ with respect to the ideal $\mathcal{J}_{y}$. Let $\mathcal{V}, u, v$ be as in 4.2.1). Since $\mathcal{F}$ is concentrated at $y$, therefore, by the construction of $f^{\sharp} \mathcal{F}$, we have $f^{\sharp} \mathcal{F}(x)=0$ for every $x$ not in $f^{-1}(y)$. This means that $f^{\sharp} \mathcal{F}(x)$ is an $\widehat{\mathcal{O}}_{y, y}$-module for every $x \in \mathcal{X}$. It follows that $f^{\sharp} \mathcal{F}=v_{*} v^{*} f^{\sharp} \mathcal{F}$. Since $v$ is adic we have $v^{*} f^{\sharp} \mathcal{F}=v^{\sharp} f^{\sharp} \mathcal{F}$, and hence $f^{\sharp} \mathcal{F}=v_{*} v^{\sharp} f^{\sharp} \mathcal{F}$ and $\lambda_{f}(\mathcal{F})=v_{*} v^{\sharp}\left(\lambda_{f}(\mathcal{F})\right.$ ). According to Proposition 4.2.2 $v^{*} \gamma_{f}^{!}(\mathcal{F})$ is an isomorphism if and only if $\gamma_{g}^{!}\left(u^{\sharp} \mathcal{F}\right)$ is an isomorphism, i.e. $v^{\sharp}\left(\lambda_{f}(\mathcal{F})\right)$ is an isomorphism if and only if $\lambda_{g}\left(u^{\sharp} \mathcal{F}\right)$ is an isomorphism. Since $\lambda_{f}(\mathcal{F})=v_{*} v^{\sharp}\left(\lambda_{f}(\mathcal{F})\right)$, it is enough to show that $\lambda_{g}\left(u^{\sharp} \mathcal{F}\right)$ is an isomorphism.

Thus we are reduced to the case $A$ is a local ring (so that $y$ has only one closed point $y$ ) and $\mathcal{F}$ is concentrated at $y$, i.e. $\mathcal{F}=i_{y} M[q], M$ a zero dimensional $A$-module. Let $\mathfrak{m}$ be the maximal ideal of $A$. We can find an exact sequence

$$
0 \longrightarrow M \longrightarrow E^{0} \longrightarrow E^{1}
$$

where $E^{0}$ and $E^{1}$ are injective $A$-modules with $\Gamma_{\mathfrak{m}} E^{i}=E^{i}$ for $i=0,1$. Let $\mathcal{G}^{i}=\left(E^{i}\right)^{\sim A}[q], i=0,1$. Note that each $\mathcal{G}^{i}$ is an object of $\operatorname{Coz}_{\Delta}(y)$. Since $f^{\sharp}$ is an exact functor (see LNS Lemma 10.2.4]) and $\lambda_{f}$ is functorial, we have a 
commutative diagram with exact rows

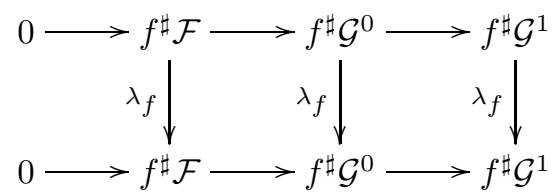

Now each $E^{i}$, being injective and zero-dimensional, is of the form $\oplus_{\alpha} K$, where $K$ is an injective hull of the residue field. By Lemma [5.3.1] $\lambda_{f}\left(\mathcal{G}^{0}\right)$ and $\lambda_{f}\left(\mathcal{G}^{1}\right)$ are isomorphisms. Hence $\lambda_{f}(\mathcal{F})$ is also an isomorphism.

\section{The Cousin of the comparison map}

In the last section we showed that if $f: X \rightarrow X$ is smooth and is a composite of compactifiable maps then $f^{\sharp}$ is a concrete model for $f^{!} \mid \mathrm{CM}(X)$. For non-smooth $f$ simple counter-examples exist showing that $f^{\sharp}$ cannot model $f^{!} \mid \mathrm{CM}(\mathcal{X})$ in general. Indeed, let $y=\operatorname{Spec} R$ where $R$ us a discrete valuation ring with residue field $k$, and let $f: X:=$ Speck $\rightarrow y$ be the natural closed immersion. Then $f^{!} k^{\sim_{R}} \simeq k \oplus k[-1]$. Now $k^{\sim_{R}} \in \operatorname{CM}(y, \Delta)$ where $\Delta$ is the codimension function on $y$ which is 0 on the closed point. But $k \oplus k[-1]$ is not Cohen-Macaulay with respect to $f^{\sharp} \Delta=0$. Note however that $E_{f^{\sharp}(\Delta)}(k \oplus k[-1]) \simeq k \simeq f^{\sharp}\left(k^{\sim_{R}}\right)$. It is worth asking-for a general map $f:\left(X, \Delta^{\prime}\right) \rightarrow(y, \Delta)$ in $\mathbb{F}_{c}^{*}$-if $f^{\sharp}$ is isomorphic to $E_{\Delta^{\prime}}\left(f^{!} Q_{X}\right)$. One of the principal results of this paper is that this is so (see Theorem 6.3.1).

6.1. Definitions and notations. Let $\left(X, \Delta^{\prime}\right) \rightarrow(y, \Delta)$ be a map in $\mathbb{F}_{c}^{*}$. Define a functor $f^{E}: \mathrm{Coz}_{\Delta}(y) \rightarrow \mathrm{Coz}_{\Delta^{\prime}}(X)$ by setting

$$
f^{E}:=E_{\Delta^{\prime}}\left(f^{!} Q y\right)
$$

The functors $f^{\sharp}$ and $f^{E}$ can be compared via the map $E_{\Delta^{\prime}}\left(\gamma_{f}^{!}\right)$. More precisely we have a functorial map

$$
\gamma_{f}^{E}: f^{\sharp} \rightarrow f^{E}
$$

defined by the composite

$$
f^{\sharp} \stackrel{\sim}{\longrightarrow} E_{\Delta^{\prime}} Q_{x} f^{\sharp} \stackrel{E_{\Delta^{\prime}}\left(\gamma_{f}^{!}\right)}{\longrightarrow} E_{\Delta^{\prime}}\left(f^{!} Q_{y}\right)=f^{E} .
$$

We will show that $\gamma_{f}^{E}$ is a functorial isomorphism. We have seen this is true when $f$ is smooth (cf. Theorem 5.3.2). Locally $f$ can be factored as a closed immersion followed by a smooth map. We therefore turn our attention to closed immersions.

6.2. Closed immersions. Suppose $f:\left(X, \Delta^{\prime}\right) \rightarrow(y, \Delta)$ is a closed immersion in $\mathbb{F}_{c}$ and suppose $\mathcal{F} \in \mathrm{Coz}_{\Delta}(y)$. Recall from [AJL2 Example 6.1.3(4)] that we have an isomorphism

$$
\bar{f}^{*} \mathbf{R} \mathcal{H o m}_{\dot{y}}\left(f_{*} \mathcal{O}_{x}, \mathcal{F}\right) \stackrel{\sim}{\longrightarrow} f^{!} \mathcal{F}
$$

induced by the universal property of $\left(f^{!}, \tau_{f}\right)$ and the map

$$
f_{*} \bar{f}^{*} \mathbf{R} \mathcal{H o m}_{\dot{y}}^{\bullet}\left(f_{*} \mathcal{O}_{x}, \mathcal{F}\right)=\mathbf{R} \mathcal{H o m} \dot{y}_{\dot{y}}\left(f_{*} \mathcal{O}_{x}, \mathcal{F}\right) \stackrel{\text { evaluation at } 1}{\longrightarrow} \mathcal{F} .
$$

Recall also from [LNS] that we have an isomorphism of Cousin complexes

$$
\bar{f}^{*} \mathcal{H o m} \dot{y}\left(f_{*} \mathcal{O}_{x}, \mathcal{F}\right) \stackrel{\sim}{\longrightarrow} f^{\sharp} \mathcal{F}
$$


such that

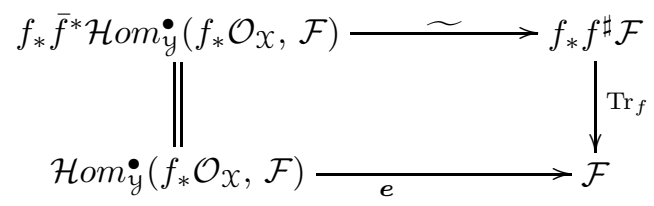

commutes where $\boldsymbol{e}$ is "evaluation at 1". Let

$$
Q_{y} \mathcal{H o m}_{\dot{y}}\left(f_{*} \mathcal{O}_{x}, \mathcal{F}\right) \longrightarrow \mathbf{R} \mathcal{H o m}_{\dot{y}}\left(f_{*} \mathcal{O}_{x}, \mathcal{F}\right)
$$

be the obvious map obtained by applying $\mathcal{H o m}_{\mathfrak{y}}\left(f_{*} \mathcal{O}_{x},-\right)$ to an $\mathcal{A}_{\text {qct }}(y)$-injective resolution $\mathcal{F} \rightarrow \mathcal{J}$ of $\mathcal{F}$. Clearly the diagram

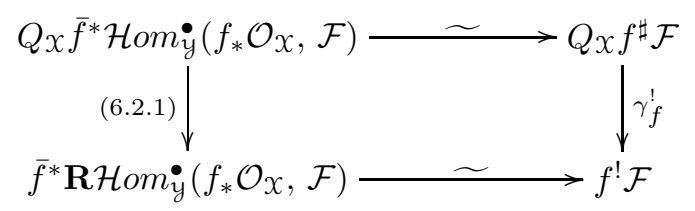

commutes, i.e. (6.2.1) is an aspect of $\gamma_{f}^{!}$. We wish to examine a similar phenomenon at a punctual level, culminating in a tractable description of functor $\mathbf{R} \Gamma_{x}\left(\gamma_{f}^{!}\right)(\simeq$ $\left.\mathbf{R} \Gamma_{f(x)}\left(f_{*} \gamma_{f}^{\prime}\right)\right)$ for a point $x \in \mathcal{X}$. To that end suppose $x \in \mathcal{X}, y=f(x), M=\mathcal{F}(y)$, $p=-\Delta(y), R=\mathcal{O}_{y, y}$ and $S=\mathcal{O}_{x, x}$. There is an isomorphism (in $\mathbf{D}^{+}(R)$ )

$$
\mathbf{R H o m}_{R}^{\bullet}(S, M[-p]) \stackrel{\sim}{\longrightarrow} \mathbf{R} \Gamma_{y} \mathbf{R} \mathcal{H} m_{\dot{y}}\left(f_{*} \mathcal{O}_{x}, \mathcal{F}\right)
$$

defined as follows. Let $\mathcal{P}$ be the open prime ideal sheaf of $\mathcal{O}_{y}$ corresponding to the point $y \in \mathcal{y}$. Then $\Gamma_{y}=\left(\Gamma_{\mathcal{P}}\right)_{y}=\Gamma_{\mathfrak{m}_{y}}(-)_{y}$. The natural map $\Gamma_{\mathcal{P}} \mathcal{F} \rightarrow \mathcal{F}$ induces a map

$$
\mathbf{R} \mathcal{H o m}_{\dot{y}}\left(f_{*} \mathcal{O}_{x}, \mathbf{R} \Gamma_{\mathcal{P}} \mathcal{F}\right) \longrightarrow \mathbf{R} \mathcal{H o m} \dot{\dot{y}}\left(f_{*} \mathcal{O}_{x}, \mathcal{F}\right)
$$

and since the source of this map is $\mathbf{R} \Gamma_{\mathcal{P}}$ stable, it factors through the map

$$
\mathbf{R} \Gamma_{\mathcal{P}} \mathbf{R} \mathcal{H o m}_{\dot{y}}\left(f_{*} \mathcal{O}_{x}, \mathcal{F}\right) \rightarrow \mathbf{R} \mathcal{H o m} \dot{\dot{y}}\left(f_{*} \mathcal{O}_{x}, \mathcal{F}\right) .
$$

It is not hard to see that the induced map

$$
\mathbf{R} \mathcal{H o m}_{\dot{y}}\left(f_{*} \mathcal{O}_{x}, \mathbf{R} \Gamma_{\mathcal{P}} \mathcal{F}\right) \rightarrow \mathbf{R} \Gamma_{\mathcal{P}} \mathbf{R} \mathcal{H o m} \dot{\dot{y}}\left(f_{*} \mathcal{O}_{x}, \mathcal{F}\right)
$$

is an isomorphism (for $f_{*} \mathcal{O}_{x}$ is coherent and $f$ is adic). Now $f_{*} \mathcal{O}_{x}$ is coherent on $y$, and so on taking stalks at $y$ we get an isomorphism

$$
\mathbf{R H o m}_{R}^{\bullet}\left(S, \mathbf{R} \Gamma_{y} \mathcal{F}\right) \stackrel{\sim}{\longrightarrow} \mathbf{R} \Gamma_{y} \mathbf{R} \mathcal{H o m} \dot{\varphi}_{\dot{y}}\left(f_{*} \mathcal{O}_{x}, \mathcal{F}\right) .
$$

Since $\mathbf{R} \Gamma_{y} \mathcal{F}=M[p]$, we obtain the map [6.2.3).

Next, let

$$
Q_{R} \operatorname{Hom}_{R}^{\bullet}(S, M) \longrightarrow \mathbf{R H o m}_{R}^{\bullet}(S, M)
$$

be the obvious map obtained by applying $\operatorname{Hom}_{R}^{\bullet}(S,-)$ to an $R$-injective resolution $M \rightarrow I^{\bullet}$ of $M$. Note that the 0th cohomology of (6.2.4) is an isomorphism. 
Proposition 6.2.5. With notations as above, the following diagram commutes:

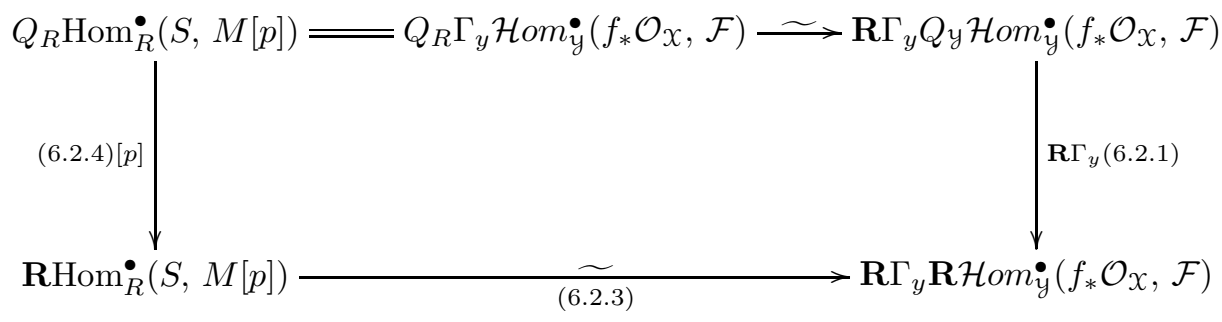

PRoOF. The proof is a straightforward unraveling of definitions. We point out that if $\mu: \mathcal{F} \rightarrow \mathcal{J}$ is a resolution of $\mathcal{F}$ by injectives in $\mathcal{A}_{\mathrm{qct}}(\mathrm{y})$ then $\Gamma_{y}(\mu): M[p] \rightarrow$ $\Gamma_{y}(\mathcal{J})$ is an injective resolution of $M[p]$. We leave the details to the reader.

Corollary 6.2.5.1. Let $f:\left(X, \Delta^{\prime}\right) \rightarrow(y, \Delta)$ be a closed immersion in $\mathbb{F}_{c}$. Then $\gamma_{f}^{E}: f^{\sharp} \rightarrow f^{E}$ is an isomorphism.

PROOF. It is enough to show that for $x \in \mathcal{X}, \mathrm{H}_{x}^{\Delta^{\prime}(x)}\left(\gamma_{f}^{!}\right)$is an isomorphism, or equivalently $\mathrm{H}_{y}^{-p}\left(f_{*} \gamma_{f}^{!}\right)$is an isomorphism where $y=f(x)$ and $p=-\Delta(y)$. By (6.2.2) and the Proposition, this is so if and only if $\left.\mathrm{H}^{-p}(\underline{6.2 .4})[p]\right)$ is an isomorphism, i.e. if and only if $\mathrm{H}^{0}(6.2 .4)$ is an isomorphism. But $\mathrm{H}^{0}(6.2 .4$ is obviously an isomorphism.

Proposition 6.2.6. Let $f:\left(\mathcal{X}, \Delta^{\prime}\right) \rightarrow(y, \Delta)$ be a closed immersion in $\mathbb{F}_{c}$ and $\mathcal{F}$ an object in $\mathrm{Coz}_{\Delta}(y)$. Then $f^{!} \mathcal{F}$ is Cohen-Macaulay with respect to $\Delta^{\prime}$ if and only if for every $x \in X$ and every $i \neq 0$

$$
\operatorname{Ext}_{R}^{i}(S, M)=0
$$

where $M=\mathcal{F}(f(x)), S=\mathcal{O}_{x, x}$ and $R=\mathcal{O}_{y, f(x)}$.

Proof. According to 6.2.3,

$$
\mathrm{H}_{x}^{i+\Delta^{\prime}(x)}\left(f^{!} \mathcal{F}\right) \simeq \operatorname{Ext}_{R}^{i}(S, M)
$$

giving the proposition.

Proposition 6.2.7. Suppose $f:\left(X, \Delta^{\prime}\right) \rightarrow(\mathcal{Y}, \Delta)$ is a map in $\mathbb{F}_{c}^{*}$ and $\mathcal{F} \in$ $\mathrm{Coz}_{\Delta}(y)$ is a complex of injective objects of $\mathcal{A}_{\mathrm{qct}}(y)$. Then $f^{!} \mathcal{F}$ is Cohen-Macaulay with respect to $\Delta^{\prime}$.

Proof. Suppose first that $f$ is a closed immersion. For $y \in \mathcal{y}, M=\mathcal{F}(y)$ is an injective $R=\mathcal{O}_{y, y}$-module. The result follows from Proposition 6.2.6

If $f$ is not a closed immersion then $f=g h$ with $h$ a closed immersion and $g$ a smooth map in $\mathbb{F}_{c}^{*}$. By Theorem 5.3 .2 we see that $g^{!} \mathcal{F} \simeq g^{\sharp} \mathcal{F}$. If the source of $g$ is $\left(\mathcal{P}, g^{\sharp} \Delta\right)$, then the Cousin complex $g^{\sharp} \mathcal{F}$ is a complex of $\mathcal{A}_{\mathrm{qct}}(\mathcal{P})$-injectives. By what just proved in the previous paragraph, $h^{!} g^{\sharp} \mathcal{F}$ is Cohen-Macaulay. But $h^{!} g^{\sharp} \mathcal{F} \simeq f^{!} \mathcal{F}$ in $\mathbf{D}_{\mathrm{qct}}^{+}(X)$ and we are done.

6.3. General maps. We are now in a position to prove

Theorem 6.3.1. Suppose $f:\left(X, \Delta^{\prime}\right) \rightarrow(y, \Delta)$ is a map in $\mathbb{F}_{c}^{*}$. Then the functorial map

is an isomorphism.

$$
\gamma_{f}^{E}: f^{\sharp} \longrightarrow f^{E}
$$


Proof. The question is local and therefore we may assume that $f=g h$ where $h$ is a closed immersion and $g$ is a smooth map in $\mathbb{F}_{c}^{*}$. By Theorem 4.1.4(d) we have a commutative diagram

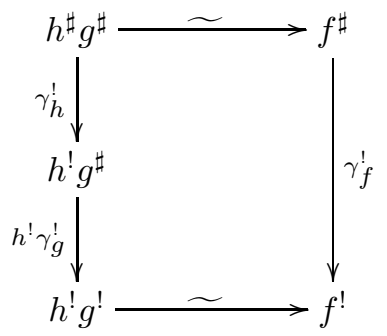

Applying $E_{\Delta^{\prime}}$ to the above diagram we get a commutative diagram

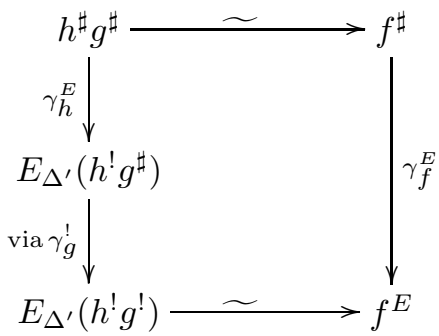

Since $h$ is a closed immersion, $\gamma_{h}^{E}$ is an isomorphism by Corollary 6.2.5.1 and by Theorem 5.3.2 $\gamma_{g}^{!}$is an isomorphism. It follows that $\gamma_{f}^{E}$ is an isomorphism.

Theorem 6.3.2. Let $\mathcal{F} \in \mathrm{Coz}_{\Delta}(y)$. The following are equivalent:

(i) The map $\gamma_{f}^{!}(\mathcal{F})$ is an isomorphism.

(ii) The twisted inverse image $f^{!} \mathcal{F}$ is Cohen-Macaulay with respect to $\Delta^{\prime}$.

Proof. Clearly (i) $\Rightarrow$ (ii). To go the other way, suppose the complex $f^{!} \mathcal{F}$ is Cohen-Macaulay. Then $\left.\gamma_{f}^{!} \mathcal{F}\right)$ is a morphism in the category $\operatorname{CM}\left(X, \Delta^{\prime}\right)$. Since the functor $E: \mathrm{CM}\left(X, \Delta^{\prime}\right) \rightarrow \mathrm{Coz}_{\Delta}(X)$ is an equivalence of categories, it is enough to prove that $E\left(\gamma_{f}^{\prime}(\mathcal{F})\right)$ is an isomorphism. This follows from the Theorem.

TheOrem 6.3.3. Let $\mathcal{F}$ be an object in $\mathrm{Coz}_{\Delta}(y)$. Then the following are equivalent

(a) $\mathcal{F}$ is a complex of $\mathcal{A}_{\mathrm{qct}}(\mathrm{y})$-injectives.

(b) $f^{!} \mathcal{F}$ is Cohen-Macaulay with respect to $f^{\sharp}(\Delta)$ for every map $f$ in $\mathbb{F}^{*}$ with target $y$.

(c) $\gamma_{f}^{!}(\mathcal{F})$ is an isomorphism for every map $f$ in $\mathbb{F}_{c}^{*}$ with target $(y, \Delta)$.

(d) $\gamma_{f}^{!}(\mathcal{F})$ is an isomorphism for every closed immersion $f$ in $\mathbb{F}_{c}$ with target $(y, \Delta)$.

(e) The map $\gamma_{f}^{!}(\mathcal{F})$ is an isomorphism for every closed immersion of the form $f:\left(X, \Delta^{\prime}\right) \rightarrow(y, \Delta)$ with $X$ an ordinary integral scheme.

Proof. From Proposition 6.2.7 we get $(\mathrm{a}) \Rightarrow(\mathrm{b})$. By Theorem 6.3.2 we have (b) $\Leftrightarrow(c)$. Clearly we have a chain of implications $(c) \Rightarrow(d) \Rightarrow(e)$. It remains to show that (e) $\Rightarrow$ (a). So suppose (e) is true. Let $y$ be a point in $y$ and $R=\mathcal{O}_{y, y}$. We have to show that $M:=\mathcal{F}(y)$ is an injective $R$-module. Since $\Gamma_{\mathfrak{m}_{R}} M=M$ it enough to show that $\mu_{i}\left(\mathfrak{m}_{R}, M\right)=0$ for $i>0$ where, for $\mathfrak{p} \in \operatorname{Spec} R, \mu_{i}(\mathfrak{p}, M)$ 
is the $i$ th Bass number of $M$ at $\mathfrak{p}$. Let $X$ be the closed integral subscheme of $y$ defined by the closure of $y$ in $y$, i.e. $X=\operatorname{Spec}\left(\mathcal{O}_{y} / \mathcal{J}\right)$ where $\mathcal{J}$ is the open prime ideal sheaf corresponding to the point $y \in y$. Let $f: X \hookrightarrow y$ be the resulting closed immersion. Let $x \in X$ be the unique point such that $f(x)=y$. Note that $x$ is the generic point of $X$ and the local ring of $X$ at $x$ is $k_{R}$. According to our hypothesis $f^{!} \mathcal{F}$ is Cohen-Macaulay with respect to $f^{\sharp} \Delta$. By Proposition 6.2.6

$$
\operatorname{Ext}_{R}^{i}\left(k_{R}, M\right)=0
$$

for $i>0$. It follows that $\mu_{i}\left(\mathfrak{m}_{R}, M\right)=0$ for $i>0$.

Remark 6.3.4. Note that by [NS Theorem 4.3.1 IV.], if $\mathcal{F}$ is a Cousin complex on $(\mathfrak{y}, \Delta)$ consisting of $\mathcal{A}_{\mathrm{qct}}(\boldsymbol{y})$ injectives (so that for $y \in \mathcal{Y}, \mathcal{F}(y)$ is a direct sum of injective hulls of the residue field at $y$ ) then $f^{\sharp} \mathcal{F}$ is complex of $\mathcal{A}_{\mathrm{qct}}(X)$ injectives for every map $f: \mathcal{X} \rightarrow \boldsymbol{y}$ in $\mathbb{F}^{*}$.

One can eliminate references to $\gamma_{f}^{!}$in the above Theorem and state it completely in terms of Grothendieck Duality and Gorenstein complexes - a notion which we now define. Let $(y, \Delta)$ be as Theorem 6.3.3 A complex $\mathcal{F}$ in $\left.\mathbf{D}_{\text {qct }}^{(} y\right)$ is said to be Gorenstein with respect to $\Delta$ if it is Cohen-Macaulay with respect to $\Delta$ and if its Cousin complex with respect to $\Delta$ consists of injective objects in $\mathcal{A}_{\text {qct }}(y)$. Theorem 6.3.3 and Remark 6.3.4 give us the following (where we decided to keep matters simple and not list all possible equivalences obtainable from Theorem 6.3 .3 and Remark 6.3.4):

TheOREm 6.3.5. Let $(y, D e)$ be as in Theorem 6.3.3 and let $\mathcal{F}$ be an object of $\mathbf{D}_{\mathrm{qct}}(\mathrm{y})$. Then the following are equivalent:

(a) $f^{!} \mathcal{F}$ is Cohen-Macaulay with respect to $f^{\sharp} \Delta$ for every map $f$ in $\mathbb{F}^{*}$ with target $y$.

(b) $f^{!} \mathcal{F}$ is Gorenstein with respect to $f^{\sharp} \Delta$ for every map $f$ in $\mathbb{F}^{*}$ with target $y$.

(c) $\mathcal{F}$ is Gorenstein with respect to $\Delta$.

\section{The Comparison map for flat morphisms}

Theorem 6.3.2 shows that $\gamma_{f}^{!}$is an isomorphism of functors if and only if $f^{!}$ takes Cohen-Macaulay objects to Cohen-Macaulay objects. This characterization, unfortunately, does not give us much information about the map $f$. In this section we prove that $\gamma_{f}^{!}$is an isomorphism of functors if and only if $f$ is flat (cf. Theorem [7.2.2].

7.1. Tor and Ext. Consider a commutative diagram in $\mathbb{F}$

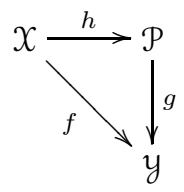

such that $f$ is smooth and $h$ is a closed immersion (so that $h$ is adic).

Proposition 7.1.1. Let $x \in X$ and let $S, R$ and $A$ be the local rings at $x$, $h(x)$ and $f(x)$ respectively. Let $k$ be the residue field of $A, \bar{R}=R \otimes_{A} k, K$ an $R$-injective hull of the residue field of $R$, and $\varphi: \widehat{A} \rightarrow \widehat{R}$ the map of complete local rings induced by $g$. 
(a) For every integer $i$ there is an isomorphism of $R$-modules

$$
\operatorname{Ext}_{R}^{i}\left(S, \varphi_{\sharp}(k)\right) \stackrel{\sim}{\longrightarrow} \operatorname{Hom}_{R}\left(\operatorname{Tor}_{i}^{R}(S, \bar{R}), K\right),
$$

i.e. $\operatorname{Ext}_{R}^{i}\left(S, \varphi_{\sharp}(k)\right)$ is the Matlis dual of the finitely generated $R$-module $\operatorname{Tor}_{i}^{R}(S, \bar{R})$.

(b) Let $i$ be an integer. Then the following are equivalent:

(i) $\operatorname{Ext}_{R}^{i}\left(S, \varphi_{\sharp}(M)\right)=0$ for every finitely generated 0 -dimensional $A$ module $M$;

(ii) $\operatorname{Ext}_{R}^{i}\left(S, \varphi_{\sharp}(M)\right)=0$ for every 0 -dimensional $A$-module $M$;

(iii) $\operatorname{Tor}_{i}^{R}(S, k)=0$.

Proof. The statements are a trifle disingenuous since both statements are trivially true if $i$ is negative. However stating matters the way we have avoids annoying trivialities later.

Let $d=\operatorname{dim} \bar{R}, \mathfrak{m}$ the maximal ideal of $R$ and $\overline{\mathfrak{m}}$ the maximal ideal of $\bar{R}$. Since $\omega_{g, x} \simeq R$ we have $R$-isomorphisms

$$
\varphi_{\sharp}(k) \simeq \mathrm{H}_{\mathfrak{m}}^{d}\left(k \otimes_{A} \omega_{g, x}\right) \simeq \mathrm{H}_{\mathfrak{m}}^{d}(\bar{R}) \simeq \mathrm{H}_{\overline{\mathfrak{m}}}^{d}(\bar{R}) .
$$

Since $\bar{R}$ is a regular local $\operatorname{ring}, \mathrm{H} \frac{d}{\mathrm{~m}}(\bar{R})$ is an $\bar{R}$-injective hull of the residue field of $\bar{R}$. This means we have an $R$-isomorphism

$$
\varphi_{\sharp}(k) \simeq \operatorname{Hom}_{R}(\bar{R}, K)
$$

since the right side is also an $\bar{R}$-injective hull of the residue field of $\bar{R}$. Let $F^{\bullet} \rightarrow S$ be an $R$-free (and hence $A$-flat) resolution of $S$. We have

$$
\begin{aligned}
\operatorname{Ext}_{R}^{i}\left(S, \varphi_{\sharp}(k)\right) & \simeq \mathrm{H}^{i}\left(\operatorname{Hom}_{R}\left(F^{\bullet}, \operatorname{Hom}_{R}(\bar{R}, K)\right)\right) \\
& \simeq \mathrm{H}^{i}\left(\operatorname{Hom}_{R}\left(F^{\bullet} \otimes_{R} \bar{R}, K\right)\right) \\
& \simeq \operatorname{Hom}_{R}\left(\mathrm{H}^{-i}\left(F^{\bullet} \otimes_{R} \bar{R}\right), K\right) \quad \text { (since } K \text { is } R \text {-injective) } \\
& \simeq \operatorname{Hom}_{R}\left(\operatorname{Tor}_{i}^{R}(S, \bar{R}), K\right),
\end{aligned}
$$

thus proving part (a).

In order to prove part (b), first note that $\operatorname{Tor}_{i}^{R}(S, \bar{R}) \simeq \operatorname{Tor}_{i}^{A}(S, k)$, since $F^{\bullet} \otimes_{R} \bar{R}=F^{\bullet} \otimes_{A} k$.

Next note that since $\operatorname{Ext}_{R}^{i}(S,-)$ commutes with direct limits, and since $\varphi_{\sharp} \simeq$ $\mathrm{H}_{\mathfrak{m}}^{d}\left(-\otimes_{A} R\right)$ commutes with direct limits, (i) and (ii) are equivalent for every module is the direct limit of finitely generated modules. Now suppose that for a given $i$ condition (ii) holds, $\operatorname{Ext}_{R}^{i}\left(S, \varphi_{\sharp}(M)\right)=0$ for every $M \in A_{\sharp}$. Since $k \in A_{\sharp}$ this implies by part (a) that the Matlis dual of the finitely generated $R$-module $\operatorname{Tor}_{i}^{R}(S, \bar{R})$ is zero. This means $\operatorname{Tor}_{i}^{R}(S, \bar{R})=0$, whence $\operatorname{Tor}_{i}^{A}(S, k)=0$.

We have to show that if $i$ satisfies condition (iii), then it satisfies condition (i). So suppose that $i$ is such that $\operatorname{Tor}_{i}^{A}(S, k)=0$, i.e. $\operatorname{Tor}_{i}^{R}(S, \bar{R})=0$. Let $F$ be the functor on finitely generated $A$-modules in $A_{\sharp}$ given by

$$
F:=\operatorname{Ext}_{R}^{i}\left(S, \varphi_{\sharp}-\right) \text {. }
$$

We have to show that $F(M)=0$ for every $M \in A_{\sharp}$ which is finitely generated. We proceed by induction on the length $\ell(M)$ of $M$. If $\ell(M)=1$, then $M \simeq k$, and by part (a) $F(k)=0$ since we have $\operatorname{Tor}_{i}^{R}(S, \bar{R})=0$. If $\ell(M)>1$ we have a short exact sequence of $A$-modules

$$
0 \longrightarrow M^{\prime} \longrightarrow M \longrightarrow M^{\prime \prime} \longrightarrow 0
$$


with $\ell\left(M^{\prime}\right), \ell\left(M^{\prime \prime}\right)<\ell(M)$. By induction hypothesis $F\left(M^{\prime}\right)=F\left(M^{\prime \prime}\right)=0$. Now $\varphi_{\sharp}$ is exact (see [LNS, Lemma 10.2.4]) and hence

$$
F\left(M^{\prime}\right) \longrightarrow F(M) \longrightarrow F\left(M^{\prime \prime}\right)
$$

is exact. It follows that $F(M)=0$.

7.2. Local cohomology and the twisted inverse image. In this subsection we examine the relationship between certain local cohomology modules associated with the twisted inverse image functor and Tor modules.

Proposition 7.2.1. Let $f:\left(\mathcal{X}, \Delta^{\prime}\right) \rightarrow(\mathcal{Y}, \Delta)$ be a map in $\mathbb{F}_{c}^{*}$. Let $x \in \mathcal{X}$, $y=f(x), S=\mathcal{O}_{x, x}, A=\mathcal{O}_{y, y}$ and $k$ the residue field of $A$. Let $p=\Delta(y)$ and $q=\Delta^{\prime}(x)$. Then for a fixed integer $i$ the following are equivalent:

(i) $\mathrm{H}_{x}^{i+q}\left(f^{!}\left(i_{y} M[-p]\right)\right)=0$ for every $M \in A_{\sharp}$.

(ii) $\mathrm{H}_{x}^{i+q}\left(f^{!}\left(i_{y} M[-p]\right)\right)=0$ for every finitely generated $A$-module in $A_{\sharp}$.

(iii) $\mathrm{H}_{x}^{i+q}\left(f^{!} \mathcal{F}\right)=0$ for every Cousin complex $\mathcal{F} \in \mathrm{Coz}_{\Delta}(y)$.

(iv) $\operatorname{Tor}_{i}^{A}(S, k)=0$

Proof. Since the statements are local in a neighborhood of $x$, we assume that $f=g h$, where $h: \mathcal{X} \rightarrow \mathcal{P}$ is a closed immersion and $g$ is smooth (and a composite of compactifiable maps). Let $R$ be the local ring at $h(x)$ and suppose $\varphi: \widehat{A} \rightarrow \widehat{R}$ is as in the statement of Proposition 7.1.1

(i) $\Leftrightarrow$ (ii). By (6.2.3) $\mathrm{H}_{x}^{i+q}\left(f^{!} i_{y} M[-p]\right)$ is isomorphic to $\operatorname{Ext}_{R}^{i}\left(S, \varphi_{\sharp}(M)\right)$. As in the proof of Proposition 7.1.1 b), by taking direct limits we see that (i) and (ii) are indeed equivalent.

(i) $\Rightarrow$ (iii). Suppose $\mathcal{F} \in \operatorname{Coz}_{\Delta}(y)$. Let $M=\mathcal{F}(y), \mathcal{G}_{1}=g^{\sharp} \mathcal{F}, \mathcal{G}_{2}=g^{\sharp}\left(i_{y} M[-p]\right)$. Let $z=h(x)$. Then $\mathcal{G}_{1}(z)=\mathcal{G}_{2}(z)=\varphi_{\sharp}(M)$. By (6.2.3) applied to the map $h$ we see that

$$
\begin{aligned}
& \mathbf{R} \Gamma_{z} \mathbf{R} \mathcal{H o m}_{\mathcal{P}}\left(h_{*} \mathcal{O}_{x}, \mathcal{G}_{1}\right) \underset{[6.2 .3}{\sim} \mathbf{R H o m}_{R}^{\bullet}\left(S, \varphi_{\sharp} M[-q]\right) \\
& \underset{\sqrt{6.2 .3}}{\stackrel{\sim}{\longrightarrow}} \mathbf{R} \Gamma_{z} \mathbf{R} \mathcal{H o m}_{\mathcal{P}}\left(h_{*} \mathcal{O}_{x}, \mathcal{G}_{2}\right) \text {. }
\end{aligned}
$$

Thus (since $Q x g^{\sharp} \simeq g ! Q_{y}$ on $\operatorname{Coz}(y)$ )

$$
\mathbf{R} \Gamma_{x} h ! g ! \mathcal{F} \simeq \mathbf{R} \Gamma_{x} h ! g^{!}\left(i_{y} M[-p]\right)
$$

i.e.

$$
\mathbf{R} \Gamma_{x} f^{!} \mathcal{F} \simeq \mathbf{R} \Gamma_{x} f^{!}\left(i_{y} M[-p]\right) .
$$

By (i) it follows that $\mathrm{H}_{x}^{i+q}\left(f^{!} \mathcal{F}\right)=0$.

(iii) $\Rightarrow$ (iv). Set $\mathcal{F}=\left(i_{y} k\right)[-p]$ and let $K$ be an $R$-injective hull of the residue field of $R$. Then

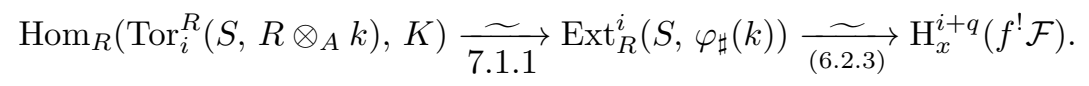

But by (iii) the last $S$-module is zero. Hence $\operatorname{Tor}_{i}^{R}\left(S, R \otimes_{A} k\right)=0$ (since its $R$ Matlis dual is zero and it is finitely generated as an $R$-module). In other words $\operatorname{Tor}_{i}^{A}(S, k) \simeq \operatorname{Tor}_{i}^{R}\left(S, R \otimes_{A} k\right)=0$.

(iv) $\Rightarrow$ (i). By Proposition 7.1.1(b), if $\operatorname{Tor}_{i}^{A}(S, k)=0$ then $\operatorname{Ext}_{R}^{i}\left(S, \varphi_{\sharp}(M)=0\right.$ for every $M \in A_{\sharp}$. By (6.2.3) this means that $\mathrm{H}_{x}^{i+q}\left(h^{!} g^{\sharp}\left(i_{y} M[-p]\right)\right)=0$ for every $M \in A_{\sharp}$. Since $g^{\sharp}\left(i_{y} M[-p]\right) \simeq g^{!}\left(i_{y} M[-p]\right)$ (cf. Theorem [5.3.2) we are done. 
Theorem 7.2.2. Let $f:\left(X, \Delta^{\prime}\right) \rightarrow(y, \Delta)$ be a map in $\mathbb{F}_{c}^{*}$. The following are equivalent:

(i) The map $\gamma_{f}^{!}: Q_{x} f^{\sharp} \rightarrow f^{!} Q_{y}$ is an isomorphism of functors.

(ii) If $\mathcal{F} \in \mathrm{Coz}_{\Delta}(\mathrm{y})$ then $f^{!} Q_{y} \mathcal{F} \in \mathrm{CM}\left(X, \Delta^{\prime}\right)$.

(iii) The functor $\left.f^{!}\right|_{\mathrm{CM}(y, \Delta)}$ takes values in $\operatorname{CM}\left(X, \Delta^{\prime}\right)$.

(iv) The map $f$ is flat.

Proof. By Theorem 6.3.2 (i) and (ii) are equivalent. Moreover (ii) and (iii) are clearly equivalent. We will show that (ii) $\Leftrightarrow\left(\right.$ iv). By Proposition $7.2 .1 f^{!} \mathcal{F}$ is Cohen-Macaulay with respect to $\Delta^{\prime}$ for every $\mathcal{F} \in \mathrm{Coz}_{\Delta}(y)$ if and only if for every $x \in X$ and every $i \neq 0 \operatorname{Tor}_{i}^{\mathcal{O}_{y, y}}\left(\mathcal{O}_{X, x}, k\right)=0$ where $y=f(x)$ and $k$ is the residue field at $y$. By $\left[\mathbf{M}\right.$ p. 174, Theorem $22.3(\mathrm{i})$ and (iii)] this is equivalent to $\mathcal{O}_{x, x}$ being flat over $\mathcal{O}_{y, y}$.

\section{The universal property of the trace}

If $f:\left(\mathcal{X}, \Delta^{\prime}\right) \rightarrow(\mathcal{Y}, \Delta)$ is a pseudo-proper map in $\mathbb{F}_{c}$ and $\rho: f_{*} \mathcal{C} \rightarrow \mathcal{F}$ a map of complexes where $\mathcal{C} \in \mathrm{Coz}_{\Delta^{\prime}}(X)$ and $\mathcal{F} \in \mathrm{Coz}_{\Delta}(\mathcal{y})$, then the resulting map $C \rightarrow f^{!} \mathcal{F}$ in $\mathbf{D}_{\text {qct }}^{+}(X)$ induces - on applying the Cousin functor $E_{\Delta}$ and the inverse of $\gamma_{f}^{E}(\mathcal{F})$ a map of Cousin complexes $\alpha(\rho): \mathcal{C} \rightarrow f^{\sharp} \mathcal{F}$. This suggests that $\left(f^{\sharp} \mathcal{F}, \operatorname{Tr}_{f}(\mathcal{F})\right)$ represents the functor $\operatorname{Hom}_{y}\left(f_{*} \mathcal{C}, \mathcal{F}\right)$ of Cousin complexes $\mathcal{C} \in \mathrm{Coz}_{\Delta^{\prime}}(\mathcal{X})$. What is required is to show that $\rho=\operatorname{Tr}_{f}(\mathcal{F}) \circ \alpha(\rho)$ and that $\delta=\alpha(\rho)$ is the only solution of the equation $\rho=\operatorname{Tr}_{f}(\mathcal{F}) \circ \delta$. This section proves these assertions and hence proves that $\left(f^{\sharp} \mathcal{F}, \operatorname{Tr}_{f}(\mathcal{F})\right)$ has a universal property giving us a duality theory for Cousin complexes.

Throughout this section we fix a morphism $f:\left(X, \Delta^{\prime}\right) \rightarrow(\mathcal{Y}, \Delta)$ in $\mathbb{F}_{c}$.

8.1. Duality for Cousin complexes. For the rest of this section the map $f$ is assumed to be pseudo-proper. For $\mathcal{C} \in \mathrm{Coz}_{\Delta^{\prime}}(\mathcal{X}), \mathcal{F} \in \mathrm{Coz}_{\Delta}(\mathcal{Y})$ and a map of complexes $\rho: f_{*} \mathcal{C} \rightarrow \mathcal{F}$ we define a map

$$
\tilde{\alpha}(\rho): \mathcal{C} \rightarrow f^{!} \mathcal{F}
$$

as the unique map such that $\tau_{f}(\mathcal{F}) \circ \mathbf{R} f_{*}(\tilde{\alpha}(\rho))=Q_{y}(\rho)$ (we are implicitly using $\mathbf{R} f_{*} \mathcal{C} \simeq Q_{y} f_{*} \mathcal{C}$. $)$ The natural isomorphism $\mathcal{C} \stackrel{\sim}{\longrightarrow} E_{\Delta^{\prime}} Q_{x} \mathcal{C}$ followed by $E_{\Delta^{\prime}}(\tilde{\alpha}(\rho))$ gives us a map

$$
\alpha^{\prime}(\rho): \mathcal{C} \rightarrow f^{E} \mathcal{F}
$$

in $\operatorname{Coz}(\mathcal{X})$. Since $\gamma_{f}^{E}$ is an isomorphism, we can define a map

$$
\alpha(\rho): \mathcal{C} \rightarrow f^{\sharp} \mathcal{F}
$$

in $\operatorname{Coz}(\mathcal{X})$ as the map satisfying $\gamma_{f}^{E}(\mathcal{F}) \circ \alpha(\rho)=\alpha^{\prime}(\rho)$.

For the rest of this section we fix the data $(\mathcal{C}, \mathcal{F}, \rho)$ where $\mathcal{C} \in \operatorname{Coz}_{\Delta^{\prime}}(X)$, $\mathcal{F} \in \mathrm{Coz}_{\Delta}(\mathcal{Y})$ and $\rho$ is a map of complexes $\rho: f_{*} \mathcal{C} \rightarrow \mathcal{F}$. Note that if $x \in \mathcal{X}$ and $y \in \mathcal{y}$ then $\rho$ induces a map $\mathcal{C}(x) \rightarrow \mathcal{F}(y)$. By [LNS Lemma 10.2.1] this map is zero unless $y=f(x)$ and $x$ is closed in $f^{-1}(y)$. For $x \in X$ closed in $f^{-1}(f(x))$ let

$$
\rho(x): \mathcal{C}(x) \rightarrow \mathcal{F}(f(x))
$$

be the map induced by $\rho: f_{*} \mathcal{C} \rightarrow \mathcal{F}$. Then

$$
\rho=\sum_{x} i_{f(x)} \rho(x)
$$


where the sum is taken over points $x$ which are closed in their fibers over $y$.

Lemma 8.1.5. Suppose $\delta \in \operatorname{Hom}_{\Delta}\left(\mathcal{C}, f^{\sharp} \mathcal{F}\right)$ is such that $\operatorname{Tr}_{f}(\mathcal{F}) \circ f_{*} \delta=\rho$. Then $\delta=\alpha(\rho)$.

Proof. By the universal property of $\left(f^{!}, \tau_{f}\right)$ we see that $\gamma_{f}^{!}(\mathcal{F}) \circ Q_{x}(\delta)=\tilde{\alpha}(\rho)$. This implies, by the definition of $\alpha^{\prime}(\rho)$ (cf. [8.1.2) $)$ and of $\gamma_{f}^{E}$ that $\gamma_{f}^{E}(\mathcal{F}) \circ \delta=\alpha^{\prime}(\rho)$. It follows that $\delta=\alpha(\rho)$.

Let $F^{p}(\mathcal{C}), G_{p}(\mathcal{C})$ be the complexes in $[\mathbf{L N S}, \S \S 10.2]$ (cf. especially the discussion immediately following the proof of Lemma 10.2.4 in Ibid..

Lemma 8.1.6. Let $\mathcal{F}^{\prime}$ and $\mathcal{C}^{\prime}$ be Cousin complexes on $(y, \Delta)$ and $\left(X, \Delta^{\prime}\right)$ respectively and suppose $\varphi \in \operatorname{Hom}_{\Delta^{\prime}}\left(\mathcal{C}^{\prime}, \mathcal{C}\right), \psi \in \operatorname{Hom}_{\Delta}\left(\mathcal{F}^{\prime}, \mathcal{F}\right)$ and $\rho^{\prime} \in \operatorname{Hom}_{y}\left(f_{*} \mathcal{C}^{\prime}, \mathcal{F}^{\prime}\right)$ are such that the diagram of complexes

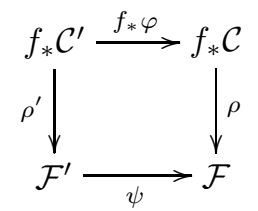

commutes. Then

$$
\alpha(\rho) \circ \varphi=f^{\sharp}(\psi) \circ \alpha\left(\rho^{\prime}\right)
$$

in $\mathrm{Coz}_{\Delta^{\prime}}(X)$.

Proof. By the universal property of $\left(f^{!}, \tau_{f}\right)$ we have $\tilde{\alpha}(\rho) \circ Q_{x} \varphi=Q_{x} f^{\sharp}(\psi) \circ$ $\tilde{\alpha}\left(\rho^{\prime}\right)$. The Lemma follows.

For a Cousin complex $\mathcal{C}$ on $\left(\mathcal{X}, \Delta^{\prime}\right)$, let $\left\{F^{p} \mathcal{C}\right\}$ and $\left\{G_{p} \mathcal{C}\right\}$ be the filtrations in [LNS $\S \S 10.2]$ (see material immediately following the proof of [Ibid., 10.2.4]). Recall from LNS $\S \S 10.2,(89)]$ that $F^{p} \mathcal{F}=f^{\sharp}\left(\sigma_{\geq p} \mathcal{F}\right)$ and $G_{p}(\mathcal{F})=f^{\sharp}\left(\sigma_{\leq p} \mathcal{F}\right)$. Note that by [LNS Lemma 10.2.1] the maps $\rho(x)$ of (8.1.4) induce maps

$$
\begin{aligned}
& \rho^{+}=\rho_{p}^{+}: f_{*} F^{p}(\mathcal{C}) \rightarrow \sigma_{\geq p} \mathcal{F} \\
& \rho^{-}=\rho_{p}^{-}: f_{*} G_{p}(\mathcal{C}) \rightarrow \sigma_{\leq p} \mathcal{F}
\end{aligned}
$$

where, for example, $\rho_{p}^{+}=\sum_{x} i_{f(x)} \rho(x)$ - the sum being taken over points $x$ such that $\Delta^{\prime}(x)=\Delta(f(x)) \geq p$.

Lemma 8.1.7. The diagram

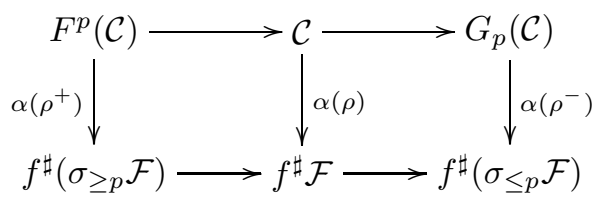

commutes.

Proof. One checks, by the definition of $\rho^{+}$, that

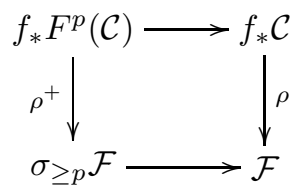


commutes, where the horizontal arrows are the obvious inclusions. The rectangle on the left in our assertion therefore commutes by Lemma 8.1.6. A similar argument gives the commutativity of the rectangle on the right.

REMARK 8.1.8. The above lemma asserts that $\alpha\left(\rho_{p}^{+}\right)=F^{p}(\alpha(\rho))$ and $\alpha\left(\rho_{p}^{-}\right)=$ $G_{p}(\alpha(\rho))$.

Proposition 8.1.9. $\operatorname{Tr}_{f}(\mathcal{F}) \circ f_{*}(\alpha(\rho))=\rho$.

Proof. Let $x \in \mathcal{X}$ be closed in its fiber. By [LNS Lemma 10.2.1] it is enough to show that

$$
\operatorname{Tr}_{f, x}(\mathcal{F}) \circ \alpha(\rho)(x)=\rho(x) .
$$

Let $p=\Delta^{\prime}(x)$. We have the identities $\rho(x)=\rho_{p}^{-}(x), \operatorname{Tr}_{f, x}(\mathcal{F})=\operatorname{Tr}_{f, x}\left(\sigma_{\leq p} \mathcal{F}\right)$ and (by Lemma 8.1.7) $\alpha(\rho)(x)=\alpha\left(\rho_{p}^{-}\right)(x)$. Thus, without loss of generality, we assume that $\mathcal{F}=\sigma_{\leq p} \mathcal{F}$ and $\mathcal{C}=G_{p}(\mathcal{C})$, by replacing $\mathcal{C}$ by $G_{p}(\mathcal{C}), \mathcal{F}$ by $\sigma_{\leq p} \mathcal{F}$ and $\rho$ by $\rho_{p}^{-}$. Now let

$$
\mathcal{C}^{\prime}:=i_{x} \mathcal{C}(x)[-p]
$$

Since $\mathcal{C}=G_{p}(\mathcal{C})$, the natural map $\varphi: \mathcal{C}^{\prime} \rightarrow \mathcal{C}$-induced by the identity map $\mathcal{C}^{\prime}(x) \rightarrow$ $\mathcal{C}(x)$-is a map of complexes. Moreover, since $\mathcal{F}=\sigma_{\leq p} \mathcal{F}$, the map $\rho(x)$ induces a map of complexes $\rho^{\prime}: \mathcal{C}^{\prime} \rightarrow \mathcal{F}$ such that $\rho^{\prime}(x)=\rho(x)$. Lemma 8.1.6 applied to $\varphi$ above and $\psi=1_{\mathcal{F}}$ gives us $\alpha(\rho)(x)=\alpha\left(\rho^{\prime}\right)(x)$. Thus in order to establish (8.1.9.1), we may (and will) assume that $\mathcal{C}=\mathcal{C}^{\prime}$ and $\rho=\rho^{\prime}$, i.e. $\mathcal{C}$ is concentrated at $x$. Let $A$ and $S$ be the completions of the local rings at $y=f(x)$ and $x$ respectively, and $h: A \rightarrow S$ the map induced by $f$. The pair $\left(\left(f^{\sharp} \mathcal{F}\right)(x), \operatorname{Tr}_{f, x}(\mathcal{F})\right)$ represents the functor $\operatorname{Hom}_{A}(M, \mathcal{F}(y))$ of 0 -dimensional $S$-modules $M$. Therefore we have a map $d: \mathcal{C}(x) \rightarrow\left(f^{\sharp} \mathcal{F}\right)(x)$ such that $\operatorname{Tr}_{f, x}(\mathcal{F}) \circ d=\rho(x)$. Since $\mathcal{C}=i_{x} \mathcal{C}(x)[-p]$, the map $d$ gives rise to a map of complexes $\delta: \mathcal{C} \rightarrow f^{\sharp} \mathcal{F}$ given by the composite

$$
\mathcal{C} \stackrel{i_{x}(d)[-p]}{\longrightarrow} i_{x}\left(\left(f^{\sharp} \mathcal{F}\right)(x)\right)[-p] \stackrel{\text { natural }}{\longrightarrow} f^{\sharp} \mathcal{F} .
$$

The second arrow is a map of complexes since the $n$th graded piece of $\mathcal{F}$ for $n \geq p$ is zero and $x$ is a point closed in its fiber with $\Delta^{\prime}(x)=p$. Clearly $\operatorname{Tr}_{f}(\mathcal{F}) \circ \delta=\rho$. By Lemma 8.1.5 we are done.

For future reference we gather the results in Lemma 8.1.5 and Proposition 8.1.9 into the following theorem.

TheOrem 8.1.10. Let $f:\left(\mathcal{X}, \Delta^{\prime}\right) \rightarrow(\mathcal{Y}, \Delta)$ be a pseudo-proper map in $\mathbb{F}_{c}$. For $\mathcal{C} \in \mathrm{Coz}_{\Delta^{\prime}}(\mathcal{X})$ and $\mathcal{F} \in \mathrm{Coz}_{\Delta}(\mathcal{y})$ the natural map

$$
\begin{aligned}
\operatorname{Hom}_{\Delta}^{\prime}\left(\mathcal{C}, f^{\sharp} \mathcal{F}\right) & \rightarrow \operatorname{Hom}_{y}\left(f_{*} \mathcal{C}, \mathcal{F}\right) \\
\delta & \mapsto \operatorname{Tr}_{f}(\mathcal{F}) \circ f_{*} \delta
\end{aligned}
$$

is a bifunctorial isomorphism. In particular, the pair $\left(f^{\sharp} \mathcal{F}, \operatorname{Tr}_{f}(\mathcal{F})\right)$ represents the functor $\operatorname{Hom}_{y}\left(f_{*} \mathcal{C}, \mathcal{F}\right)$ of Cousin complexes $\mathcal{C}$ on $\left(\mathcal{X}, \Delta^{\prime}\right)$.

Proof. Lemma 8.1.5 proves that the map $\delta \mapsto \operatorname{Tr}_{f}(\mathcal{F}) \circ f_{*} \delta$ is injective. Proposition 8.1.9 shows that it is surjective.

Theorem 8.1.10 has an interesting corollary when $f$ is a pseudo-finite map, i.e. $f$ is pseudo-proper and its fibers are finite, or equivalently, $f$ is pseudo-proper and affine. In this case, $f$ corresponds locally (on $y$ ) to a homomorphism $\varphi:(R, I) \rightarrow$ 
$(S, J)$ of adic rings (i.e. $\varphi(I) \subset J)$ and $S / J$ is a finite $R$-module. Let $\mathcal{U}=\operatorname{Spf}(R, I)$ and $\mathcal{V}=f^{-1}(\mathcal{U})=\operatorname{Spf}(S, J)$. As in AJL2, $\S \S 2.1$ ] set

$$
\operatorname{Hom}_{R, J}(S, F):=\Gamma_{J} \operatorname{Hom}_{R}(S, F)
$$

where $F=\Gamma(\mathcal{U}, \mathcal{F})\left(\mathcal{F} \in \mathrm{Coz}_{\Delta}(\mathcal{y})\right.$ as in Theorem 8.1.10). The complex $\operatorname{Hom}_{R, J}(S, F)$ can also be interpreted as the complex of $S$-modules of continuous $R$-maps from $S$ to $F$ when $S$ is $J$-adically topologized and $F$ is discrete. Moreover

$$
\operatorname{Hom}_{R, J}(S, F)=\underset{n}{\lim _{n}} \operatorname{Hom}_{R}\left(S / J^{n}, F\right)
$$

by standard 3-lemma arguments. Since $\mathcal{O}_{x}$ is coherent and $\mathcal{F}$ is a Cousin complex the complexes $\operatorname{Hom}_{R, J}(S, F)^{\sim}$ can be patched as $\mathcal{U}=\operatorname{Spf} R$ varies over an affine open cover of $y$ to give a complex $f^{b} \mathcal{F}$ (cf. [LNS Lemma 2.3.5(iii)] applied to the torsion modules $\operatorname{Hom}_{R, J}(S, \mathcal{F}(y))$ for $\left.y \in \mathcal{y}\right)$.

If $\mathcal{J}$ is a defining ideal for $X$ and $X_{n}=\left(X, \mathcal{O}_{X} / \mathcal{J}^{n}\right)$ then by 8.1.10.1)

$$
f^{\mathrm{b}} \mathcal{F}=\underset{n}{\lim _{n}} i_{n *} f_{n}^{\mathrm{b}} \mathcal{F}
$$

where $i_{n}: X_{n} \hookrightarrow X$ is the natural closed immersion and $f_{n}: X_{n} \rightarrow y$ the resulting finite (= adic and pseudo-finite) map. It is not hard to see that $f_{n}^{b} \mathcal{F}$ is Cousin on $\left(X_{n}, f_{n}^{\sharp} \Delta\right)$, whence $f^{b} \mathcal{F}$ is Cousin on $\left(\mathcal{X}, \Delta^{\prime}\right)$. Moreover, for $x \in \mathcal{X}($ with $y=f(x))$ one has

$$
\left(f^{b} \mathcal{F}\right)(x)=\Gamma_{\mathfrak{m}_{\widehat{S}}} \operatorname{Hom}_{\widehat{R}}(\widehat{S}, \mathcal{F}(y))=\operatorname{Hom}_{\widehat{R}}^{c}(\widehat{S}, \mathcal{F}(y))
$$

where $\widehat{R}$ (resp. $\widehat{S}$ ) is the completion of the local ring at $y$ (resp. $x$ ) and the superscript "c" over the Hom on the right refers to continuous $\widehat{R}$-maps with $\widehat{S}$ having the $\mathfrak{m}_{\widehat{S}}$-adic topology and $\mathcal{F}(y)$ having the discrete topology. The relation 8.1.10.3) is obtained by using (8.1.10.2) to reduce to the case where $f$ is finite where the relation is not hard to establish. Using [Hu1 §7] (see also (2.2.2) ) we have an isomorphism of $\widehat{S}$-modules

$$
\Theta(x)=\Theta_{f}(x):\left(f^{\sharp} \mathcal{F}\right)(x) \stackrel{\sim}{\longrightarrow} \operatorname{Hom}_{\widehat{R}}^{c}(\widehat{S}, \mathcal{F}(y))=\left(f^{\mathrm{b}} \mathcal{F}\right)(x)
$$

characterized by the relation

$$
\boldsymbol{e}(x) \circ \Theta(x)=\operatorname{Tr}_{f}(\mathcal{F})(x)
$$

where $\boldsymbol{e}(x): \operatorname{Hom}_{\widehat{R}}^{c}(\widehat{S}, \mathcal{F}(y)) \rightarrow \mathcal{F}(y)$ is "evaluation at 1 ". That this is a characterization is readily seen by observing that $\left(f^{\sharp} \mathcal{F}(x), \operatorname{Tr}_{f}(\mathcal{F})(x)\right)$ and $\left(f^{b} \mathcal{F}(x), \boldsymbol{e}(x)\right)$ represent the same functor, viz. the functor $\operatorname{Hom}_{\widehat{R}}(N, \mathcal{F}(y))$ of zero-dimensional $\widehat{S}$-modules $N$.

From the $\Theta(x)$ we get in an obvious way a map of graded $\mathcal{O}_{x}$-modules

$$
\Theta_{f}(\mathcal{F}): f^{\sharp} \mathcal{F} \stackrel{\sim}{\longrightarrow} f^{b} \mathcal{F}
$$

which is functorial in $\mathcal{F} \in \mathrm{Coz}_{\Delta}(y)$. A consequence of Theorem 8.1.10 is the following:

Corollary 8.1.11. Let $f:\left(X, \Delta^{\prime}\right) \rightarrow(y, \Delta)$ be a pseudo-finite map in $\mathbb{F}_{c}$ and $\mathcal{F}$ a Cousin complex on $(\mathcal{Y}, \Delta)$. Then the graded map $\Theta_{f}(\mathcal{F})$ above is a map of complexes. 
Proof. By (8.1.10.2) it is enough to assume $f$ is finite. We have a map $\boldsymbol{e}: f_{*} f^{b} \mathcal{F} \rightarrow \mathcal{F}$ given by "evaluation at 1 " (note that $f_{*} f^{b} \mathcal{F}=\mathcal{H} o m_{\dot{y}}\left(f_{*} \mathcal{O}_{x}, \mathcal{F}\right)$ ). Clearly $\left(f^{b} \mathcal{F}, \boldsymbol{e}\right)$ represents the same functor that $\left(f^{\sharp} \mathcal{F}, \operatorname{Tr}_{f}(\mathcal{F})\right)$ does. This results in an isomorphism

$$
\Theta^{\prime}: f^{\sharp} \mathcal{F} \stackrel{\sim}{\longrightarrow} f^{b} \mathcal{F}
$$

such that $e \circ f_{*}\left(\Theta^{\prime}\right)=\operatorname{Tr}_{f}(\mathcal{F})$. It follows that for a point $x \in \mathcal{X}, \Theta^{\prime}(x)=\Theta_{f}(\mathcal{F})(x)$, whence $\Theta^{\prime}=\Theta_{f}(\mathcal{F})$.

\section{Variants}

In this section we construct a variant of $-!$ on the full subcategory of $\mathbb{F}$ consisting of schemes which admit a bounded residual complex (cf. [LNS $\S \S 9.1$ ], Y 5.9] and, for related matters, AJL2 $\S \S 2.5])$. For every such scheme, the associated category $X^{(!)}$is a full subcategory $\widetilde{\mathbf{D}}_{\mathrm{c}}^{*}(X)$ of $\widetilde{\mathbf{D}}_{\mathrm{qc}}^{+}(X)$. The method we use is the Grothendieck's original method via residual complexes developed in [Hrt], but with our canonical Cousin complex valued pseudofunctor $-\sharp$ Cousin complexes (more precisely, its "restriction" to residual complexes) in place of $-^{\boldsymbol{\Delta}}$ of $\left.\mathbf{H r t}\right]$. Moreover, we are dealing with formal schemes rather than ordinary schemes and hence there is a need to retell the story, albeit in an abbreviated form. The reader is advised to look at the very careful account given by Conrad in C. Chapter 3] (especially $\S 3.3$ and $\S 3.4$ ) to flesh out missing details in what follows.

9.1. Preliminaries. Let $y$ be a noetherian formal scheme. Suppose $y$ admits a bounded residual complex $\mathcal{R}$ [LNS $\S \S 9.1]^{8}$. By [NS 9.2.2 (ii) and (iii)] $\mathcal{R}$ is a $t$-dualizing complex in the sense of AJL2 Definition 2.5.1]. By [Y] Theorem 5.6], if $\mathcal{R}^{\prime}$ is another residual complex on $y$ then $\mathcal{R}^{\prime}=\mathcal{R} \otimes \mathcal{L}[n]$ for an invertible $\mathcal{O}_{y-}$ module $\mathcal{L}$ and an integer $n$. The residual complex $\mathcal{R}$ induces a codimension function $\Delta_{\mathcal{R}}$ on $y$; for a point $y$ in $y, \Delta_{\mathcal{R}}(y)$ is the unique integer $p$ such that $\mathrm{H}_{y}^{\prime p}(\mathcal{R})$ is non-zero.

For any $\mathcal{E} \in \mathbf{D}(y)$ set

$$
\mathcal{D}_{\mathcal{R}}(\mathcal{E}):=\mathbf{R H o m}^{\bullet}(\mathcal{E}, \mathcal{R}) .
$$

Now, $\mathcal{R}$ is a complex of $\mathcal{A}_{\text {qct }}(y)$-injectives (cf. [LNS 9.1.3] and [LNS 2.3.6 (ii)]), and therefore, if $\mathcal{E} \in \mathbf{D}_{\text {qct }}^{+}(\boldsymbol{y})$ we may, and will, make the identification

$$
\mathcal{D}_{\mathcal{R}}(\mathcal{E})=\mathcal{H o m}^{\bullet}(\mathcal{E}, \mathcal{R})
$$

(cf. AJL2 Proposition 5.3.1]). We refer the reader to [AJL2, $\S \S 2.5$ - especially (a) and (c) of Proposition 2.5.8 - for further details on $\mathcal{D}_{\mathcal{R}}$.

9.2. Twisted inverse image via residual complexes. Let $\mathbb{F}^{r}$ be the full subcategory of $\mathbb{F}$ consisting of schemes which admit bounded residual complexes. For $y \in \mathbb{F}^{r}$ let $\mathbf{D}_{\mathrm{c}}^{*}(y)$ and $\widetilde{\mathbf{D}}_{\mathrm{c}}^{*}(y)$ be as in Subsection 1.1 and set

$$
y^{(!)}:=\widetilde{\mathbf{D}}_{\mathrm{c}}^{*}(y) .
$$

For the rest of this subsection we fix maps

$$
\mathcal{V} \stackrel{h}{\rightarrow} \mathcal{W} \stackrel{g}{\rightarrow} x \stackrel{f}{\rightarrow} y
$$

in $\mathbb{F}^{r}$.

\footnotetext{
${ }^{8}$ This forces $y$ to have finite Krull dimension.
} 
Suppose $\mathcal{R}$ is a residual complex on $y$. Define

$$
f_{\mathcal{R}}^{(!)}: y(!) \longrightarrow x^{(!)}
$$

by setting

$$
f_{\mathcal{R}}^{(!)}:=\mathcal{D}_{f^{\sharp} \mathcal{R}} \circ \mathbf{L} f^{*} \circ \mathcal{D}_{\mathcal{R}} \circ \mathbf{R} \Gamma_{y}^{\prime} .
$$

This functor takes values in $X^{(!)}$for the following reasons; (a) $\mathcal{E} \in y^{(!)} \Rightarrow \mathbf{R} \Gamma_{y}^{\prime} \mathcal{E} \in$ $\mathbf{D}_{\mathrm{c}}^{*}(y) \cap \mathbf{D}^{+}(\mathrm{y})$ by definition of $y(!) ;(b) \mathcal{E} \in \mathbf{D}_{\mathrm{c}}^{*}(y) \cap \mathbf{D}^{+}(y) \Rightarrow \mathcal{D}_{\mathcal{R}}(\mathcal{E}) \in \mathbf{D}_{\mathrm{c}}^{-}(y)$ by AJL2 Proposition 2.5.8(a)], (c) $\mathcal{G} \in \mathbf{D}_{\mathrm{c}}^{-}(\mathrm{y}) \Rightarrow \mathbf{L} f^{*} \mathcal{G} \in \mathbf{D}_{\mathrm{c}}^{-}(X)$ and (d) $\mathcal{F} \in$ $\mathbf{D}_{\mathrm{c}}^{-}(X) \Rightarrow \mathcal{D}_{f^{\sharp} \mathcal{R}}(\mathcal{F}) \in \mathbf{D}_{\mathrm{c}}^{*}(X) \cap \mathbf{D}^{+}(X)$ by [AJL2 Proposition 2.5.8 (b)].

Defining a pseudofunctor ${ }^{(!)}$on $\mathbb{F}^{r}$ is now a formal process given in detail in C] §3.3]. We point out the main steps.

1) If $\mathcal{R}^{\prime}$ is another residual complex on $\boldsymbol{y}$, then as in [C] p. 135, (3.3.12)] we have a comparison map (stemming from $\mathcal{R}^{\prime} \simeq \mathcal{R} \otimes \mathcal{L}[n]$ for some invertible sheaf $\mathcal{L}$ which is compatible with a similar relation between $f^{\sharp} \mathcal{R}^{\prime}$ and $f^{\sharp} \mathcal{R}$ via $f^{*} \mathcal{L}[n]$ )

$$
\phi_{\mathcal{R}, \mathcal{R}^{\prime}}=\phi_{f, \mathcal{R}, \mathcal{R}^{\prime}}: f_{\mathcal{R}^{\prime}}^{(!)} \longrightarrow f_{\mathcal{R}}^{(!)}
$$

which satisfies the cocycle condition for three residual complexes $\mathcal{R}, \mathcal{R}^{\prime}, \mathcal{R}^{\prime \prime}$ given in C. p. 135, (3.3.13)]. Standard techniques give a well defined functor

$$
f^{(!)}: y^{(!)} \longrightarrow x^{(!)}
$$

together with isomorphisms

$$
\theta_{\mathcal{R}}=\theta_{f, \mathcal{R}}: f_{\mathcal{R}}^{(!)} \stackrel{\sim}{\longrightarrow} f^{(!)}
$$

satisfying

$$
\phi_{\mathcal{R}, \mathcal{R}^{\prime}}=\theta_{\mathcal{R}}^{-1} \circ \theta_{\mathcal{R}^{\prime}}
$$

2) As in [C] p. 136, (3.3.15)] the isomorphism $C_{g, f}^{\sharp}: g^{\sharp} f^{\sharp} \mathcal{R} \stackrel{\sim}{\longrightarrow}(f g)^{\sharp} \mathcal{R}$ gives an isomorphism

$$
C_{g, f, \mathcal{R}}^{(!)}: g_{f^{\sharp} \mathcal{R}}^{(!)} f_{\mathcal{R}}^{(!)} \stackrel{\sim}{\longrightarrow}(f g)_{\mathcal{R}}^{(!)}
$$

in such a way that "associativity" holds — the last because $-^{\sharp}$ is a pseudofunctor. One checks that the isomorphism

$$
C_{g, f}^{(!)}: g^{(!)} f^{(!)} \stackrel{\sim}{\longrightarrow}(f g)^{(!)}
$$

defined by $C_{g, f, \mathcal{R}}^{(!)}, \theta_{f, \mathcal{R}}, \theta_{g, f^{\sharp} \mathcal{R}}$ and $\theta_{f g, \mathcal{R}}$ is independent of $\mathcal{R}$.

This gives the required (pre)-pseudofunctor on $\mathbb{F}^{r}$.

9.3. Comparison of the two twisted inverse images. We will use the discussion in Subsection 3.3 to show that $-^{!}$and $-(!)$agree whenever both are defined (cf. Theorem 9.3.10). With that in mind we examine the two theories for open immersions and for pseudo-proper maps.

1) Suppose $f: X \rightarrow y$ is an open immersion in $\mathbb{F}^{r}$. Let $\mathcal{R}$ be a residual complex on $y$. Then $f^{\sharp} \mathcal{R}=f^{*} \mathcal{R}$. Moreover $f^{!}=f^{*} \Gamma_{y}^{\prime}=\Gamma_{x}^{\prime} f^{*}$. If $\mathcal{F} \in y^{(!)}$we have the 
following sequence of isomorphisms

$$
\begin{aligned}
f_{\mathcal{R}}^{(!)} \mathcal{F} & =\mathcal{H o m}_{\dot{y}}\left(f^{*} \mathcal{H o m} \dot{x}\left(\Gamma_{y}^{\prime} \mathcal{F}, \mathcal{R}\right), f^{*} \mathcal{R}\right) \\
& =\mathcal{H o m}_{\dot{y}}\left(\mathcal{H o m}_{\dot{y}}\left(f^{*} \Gamma_{\mathcal{F}}^{\prime}, f^{*} \mathcal{R}\right), f^{*} \mathcal{R}\right) \\
& =\mathcal{D}_{f^{*} \mathcal{R}} \mathcal{D}_{f^{*} \mathcal{R}} f^{*} \Gamma_{\mathcal{F}}^{\prime} \\
& \stackrel{\sim}{\longrightarrow} f^{*} \Gamma_{y}^{\prime} \mathcal{F} \\
& =f^{!} \mathcal{F}
\end{aligned}
$$

Let $\Phi_{f, \mathcal{R}}: f_{\mathcal{R}}^{(!)} \mathcal{F} \stackrel{\sim}{\longrightarrow} f^{!} \mathcal{F}$ be the above composite. It is easy to verify that that

$$
\Phi_{f}:=\Phi_{f, \mathcal{R}} \circ \theta_{\mathcal{R}}^{-1}:\left.f^{(!)} \stackrel{\sim}{\longrightarrow} f^{!}\right|_{y}(!)
$$

is independent of $\mathcal{R}$.

2) Suppose $f: X \rightarrow Y$ is a map in $\mathbb{F}^{r}$ which is pseudo-proper. Let $\mathcal{R}$ be a residual complex on $y$ and let $\mathcal{F}$ be an object in $y(!)$. One then has the following sequence of maps - the first arrow arising from the adjoint pair $\left(\mathbf{L} f^{*}, \mathbf{R} f_{*}\right)$ :

$$
\begin{aligned}
& \mathbf{R} f_{*} f^{(!)} \mathcal{F} \longrightarrow \mathbf{R} f_{*} \mathbf{R} \mathcal{H o m}{ }_{\mathcal{X}}\left(\mathbf{L} f^{*} \mathcal{D}_{\mathcal{R}} \Gamma_{\mathcal{y}}^{\prime} \mathcal{F}, f^{\sharp} \mathcal{R}\right) \\
& \longrightarrow \mathbf{R} \mathcal{H o m}_{\dot{y}}\left(\mathcal{D}_{\mathcal{R}} \Gamma_{y}^{\prime} \mathcal{F}, \mathbf{R} f_{*} f^{\sharp} \mathcal{R}\right) \\
& \stackrel{\text { via } \operatorname{Tr}_{f}}{\longrightarrow} \mathbf{R} \mathcal{H} m_{\dot{y}}\left(\mathcal{D}_{\mathcal{R}} \Gamma_{y}^{\prime}, \mathcal{R}\right)=\mathcal{D}_{\mathcal{R}} \mathcal{D}_{\mathcal{R}} \Gamma_{y}^{\prime} \mathcal{F} \\
& \longrightarrow \Gamma_{y}^{\prime} \mathcal{F} \\
& \stackrel{\text { nat'l }}{\longrightarrow} \mathcal{F}
\end{aligned}
$$

The above composite gives a trace map

$$
\tau_{\mathcal{R}}(\mathcal{F})=\tau_{f, \mathcal{R}}(\mathcal{F}): \mathbf{R} f_{*} f^{(!)} \mathcal{F} \longrightarrow \mathcal{F} .
$$

If $g: \mathcal{W} \rightarrow X$ is a second pseudo-proper map, one checks using (2.2.6) (i.e. the transitivity property of traces on Cousin complexes) that the following relation holds

$$
\tau_{\mathcal{R}, f g}=\tau_{f, \mathcal{R}} \circ \tau_{f^{\sharp} \mathcal{R}} \circ \mathbf{R} f_{*} \mathbf{R} g_{*}\left(C_{g, f, \mathcal{R}}^{(!)}\right)^{-1} .
$$

Define

$$
\tau_{f}^{r}:=\tau_{f, \mathcal{R}} \circ \mathbf{R} f_{*}\left(\theta_{\mathcal{R}}^{-1}\right) .
$$

Since $\operatorname{Tr}_{f}(\mathcal{R})$ is compatible with Zariski localizations of $y$, functorial with respect to maps of residual complexes with the same codimension function (whence compatible with tensoring $\mathcal{R}$ by an invertible sheaf) and compatible with translations of residual complexes, therefore one checks that

$$
\tau_{f}^{r}: \mathbf{R} f_{*} f^{(!)} \rightarrow \mathbf{1}_{\mathbf{y}(!)}
$$

is independent of the residual complex $\mathcal{R}$. Since a residual complex on $y$ is a complex of $\mathcal{A}_{\text {qct }}(y)$-injectives therefore Theorem 6.3.3 applies and we make the identifications

$$
f^{!} \mathcal{R}=f^{\sharp} \mathcal{R} \quad \tau_{f}(\mathcal{R})=\operatorname{Tr}_{f}(\mathcal{R})
$$

By AJL2, 2.5.12 and 6.1.5(b)] and 9.3.6) we get an isomorphism

$$
\Phi_{f}:\left.f^{(!)} \stackrel{\sim}{\longrightarrow} f^{!}\right|_{y(!)}
$$


- the map $\Phi_{f}$ being the unique map arising from the universal property of the pair $\left(f^{!}, \tau_{f}\right)$ for which the relation

$$
\tau_{f}^{r}=\tau_{f} \circ \mathbf{R} f_{*} \Phi_{f}
$$

holds.

If $g: \mathcal{W} \rightarrow \mathcal{X}$ is a second pseudo-proper map then the transitivity relation 9.3.4 above gives us a commutative diagram

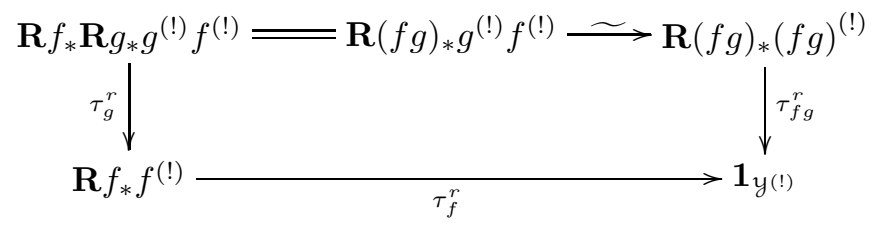

We are now in a position to state the following Theorem, which can be reformulated as stating that appropriate restrictions of the pseudofunctors $-!$ and $-{ }^{(!)}$are isomorphic.

THEOREM 9.3.10. There is a unique family of isomorphisms

$$
\Phi_{f}:\left.f^{(!)} \stackrel{\sim}{\longrightarrow} f^{!}\right|_{y(!)},
$$

one for each map $f: X \rightarrow y$ in $\mathbb{F}^{r} \cap \mathbb{F}^{*}$ such that

(a) If $f$ is pseudo-proper and $\mathcal{F} \in y^{(!)}$then $\Phi_{f}$ is the map (9.3.7), i.e., it is the unique map satisfying

$$
\tau_{f}^{r}(\mathcal{F})=\tau_{f}(\mathcal{F}) \circ \Phi_{f}(\mathcal{F}) .
$$

(b) If $f$ is an open immersion then $\Phi_{f}$ is the isomorphism (9.3.2).

(c) If $g: \mathcal{W} \rightarrow \mathcal{X}$ is a second map in $\mathbb{F}^{r} \cap \mathbb{F}^{*}$ then the diagram

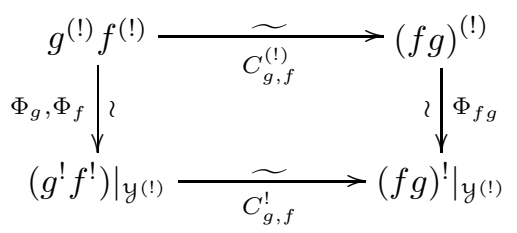

commutes.

(d) The map $\Phi_{f}$ is compatible with Zariski localizations of $y$.

Proof. We wish to use Theorem 3.3.4 To that end, for $X \in \mathbb{F}^{r} \cap \mathbb{F}^{*}$ let

$$
S_{X}: X^{(!)}=\widetilde{\mathbf{D}}_{\mathrm{c}}^{*}(X) \rightarrow X^{!}=\widetilde{\mathbf{D}}_{\mathrm{qc}}^{+}(X)
$$

be the natural inclusion. Here is the dictionary to help us pass from Subsection 3.3 to the situation we are now in. The subcategory $\overline{\mathbb{G}}$ in Subsection 3.3 is, for us, $\mathbb{F}^{r} \cap \mathbb{F}^{*}$, the pseudofunctor $-^{\natural}$ is $-^{(!)}$and the maps $\gamma_{f}$ for $f \in \mathbf{P} \cup \mathbf{F}$ are the maps $\Phi_{f}$ of (9.3.2) and (9.3.7). Checking diagram (3.3.1) commutes amounts to checking that 9.3.10.1) commutes when $f$ and $g$ are either both open or both pseudo-proper.

In view of (9.3.9), the diagram (9.3.10.1) commutes whenever $f$ and $g$ are both pseudo-proper. If $f$ and $g$ are both open immersions then from the definition in 9.3.1) it is clear that 9.3.10.11 commutes.

Next suppose $f: X \rightarrow y$ is a pseudo-proper map in $\mathbb{F}^{r} \cap \mathbb{F}^{*}$ and suppose $u: \mathcal{U} \rightarrow \mathcal{y}$ is an open immersion. Consider the resulting fiber square diagram 
(3.3.2). Pick a residual complex $\mathcal{R}$ on $y$. Then $\operatorname{Tr}_{f}(\mathcal{R})$ is compatible with open immersions into $y$ and hence so is $\tau_{f}^{r}$. It follows that (3.3.3) commutes.

REMARK 9.3.11. As we mentioned earlier, the theorem is a way of saying that the pseudofunctors $-^{(!)}$and $-^{!}$are isomorphic when each is appropriately restricted to the "domain" where both are meaningful. The reformulation is a little awkward in view of the fact that there are numerous ways of "restricting" pseudofunctors since we are dealing with families of categories indexed by yet another category. We state what is needed briefly. Suppose $\overrightarrow{-}^{(!)}$is the pseudofunctor obtained by restricting - (!) to $\mathbb{F}^{r} \cap \mathbb{F}^{*}$ and $\rightarrow$ ! the pseudofunctor obtained on $\mathbb{F}^{r} \cap \mathbb{F}^{*}$ via restricting - ! and by setting $\vec{X}^{!}=X^{(!)}$(there are two types of restrictions inherent here). Then Theorem 9.3 .10 asserts that $\vec{\beth}^{(!)}$is isomorphic to $\vec{\longrightarrow}$ !

TheOrem 9.3.12. Let $f:\left(X, \Delta^{\prime}\right) \rightarrow(\mathcal{Y}, \Delta)$ be a map in $\mathbb{F}_{c}^{r}$, and $\Delta$ a codimensions function on $y$. Then $\left.f^{(!)}\right|_{\mathrm{CM}^{*}(y, \Delta)}$ takes values in $\mathrm{CM}^{*}\left(X, \Delta^{\prime}\right)$ if and only if $f$ is flat.

Proof. The question is local and locally $f$ can be factored as a composite of pseudo-proper maps and open immersions (cf. [LNS Lemma 2.4.3]). Therefore, without loss of generality, we assume that $f$ is also in $\mathbb{F}_{c}^{*}$. If $f$ is flat then Theorem 9.3.10 and Theorem 7.2.2 imply that $f^{(!)} \mathcal{F}$ is in $\mathrm{CM}^{*}\left(\mathcal{X}, \Delta^{\prime}\right)$ if $\mathcal{F} \in \mathrm{CM}^{*}(\mathcal{y}, \Delta)$. For the converse we need to argue with a little care, for Theorem 7.2 .2 requires one to test $f^{!} \mathcal{F}$ for every $\mathcal{F} \in \mathrm{CM}(\mathcal{Y}, \Delta)$, whereas we are restricting our $\mathcal{F}$ 's to lie in $\mathrm{CM}^{*}(y, \Delta)$. So suppose $f^{(!)} \mathcal{F} \in \mathrm{CM}^{*}\left(X, \Delta^{\prime}\right)$ whenever $\mathcal{F} \in \mathrm{CM}^{*}(y, \Delta)$. Let $x \in X, S=\mathcal{O}_{x, x}, y=f(x)$ and $A=\mathcal{O}_{y, y}$. By restricting to an open neighborhood of $x$ if necessary, we may assume that $f=g h$ where $X \stackrel{h}{\rightarrow} \mathcal{P}$ is a closed immersion and $\mathcal{P} \stackrel{g}{\rightarrow} y$ is a smooth map in $\mathbb{F}^{*}$. Let $R=\mathcal{O}_{\mathcal{P}, h(x)}$ and $z=h(x)$. As in Proposition 7.1.1 let $k=k_{A}, K$ an $R$-injective hull of $k_{R}$ and $\varphi: \widehat{A} \rightarrow \widehat{R}$ the natural map induced by $g$. In what follows we will be applying Theorem 9.3 .10 to the maps $f$, $g$ and $h$ without comment since all three of them are morphisms in $\mathbb{F}^{r} \cap \mathbb{F}^{*}$. Let $\mathcal{J} \subset \mathcal{O} y$ be the open prime ideal sheaf corresponding to the point $y$. Let $\mathcal{R}$ be a residual complex in $\mathrm{Coz}_{\Delta}(y)$. Set

$$
\mathcal{F}:=\mathcal{H o m}_{\mathfrak{y}}\left(\mathcal{O}_{y} / \mathcal{J}, \mathcal{R}\right) .
$$

Then $Q_{y} \mathcal{F} \in \mathrm{CM}^{*}(y, \Delta)$. Hence by our hypothesis $f^{!} \mathcal{F} \in \mathrm{CM}^{*}\left(X, \Delta^{\prime}\right)$. Therefore

$$
\mathrm{H}_{x}^{i+\Delta^{\prime}(x)}\left(f^{!} \mathcal{F}\right)=0
$$

for $i \neq 0$. By (6.2.3) and the fact that $f^{!} \mathcal{F} \simeq h^{!} g^{\sharp} \mathcal{F}$ this amounts to saying

$$
\operatorname{Ext}_{R}^{i}\left(S,\left(g^{\sharp} \mathcal{F}\right)(z)\right)=0
$$

for $i>0$. By Proposition 7.1.1 (b) this means that $\operatorname{Tor}_{i}^{A}(S, k)=0$ for $i>0$. Hence we are done by $\mathbf{M}$ p. 174, Thm.22.3, (i) and (iii)].

The following result follows from Theorem 6.3.3 and Theorem 9.3.10, and the fact that we may, in each case, Zariski localize the source and the target.

Theorem 9.3.13. Let $(y, \Delta) \in \mathbb{F}_{c}^{r}$ and let $\mathcal{F} \in \mathrm{Coz}_{\Delta}(y)$. Then the following are equivalent

(a) $\mathcal{F}$ is a complex of $\mathcal{A}_{\mathrm{qct}}$-injectives;

(b) $f^{(!)} \mathcal{F} \in \mathrm{CM}^{*}\left(X, \Delta^{\prime}\right)$ for every morphism $\left(X, \Delta^{\prime}\right) \stackrel{f}{\rightarrow}(\mathcal{Y}, \Delta)$ in $\mathbb{F}_{c}^{r}$; 
(c) $f^{(!)} \mathcal{F} \in \mathrm{CM}^{*}\left(X, \Delta^{\prime}\right)$ for every closed immersion $\left(X, \Delta^{\prime}\right) \stackrel{f}{\rightarrow}(\mathrm{y}, \Delta)$ such that $X$ is an integral ordinary scheme.

ACKNOWLEDGMENTs . This work has been a long time in the making, and from its very beginning in 1995, Joe Lipman has been a constant source of stimulation and encouragement. I also wish to thank Suresh Nayak for stimulating conversations and for being sensitive to the needs of this paper while Lipman, he and I were working on $[\mathbf{L N S}$. 


\section{References}

[AJL1] L. Alonso Tarrío, A. Jeremías López and J. Lipman, Local homology and cohomology on schemes, Ann. Scient. Éc. Norm. Sup. 30 (1997), 1-39. See also Correction, on page 879 of vol. 2 of the Collected Papers of Joseph Lipman, Queen's Papers in Pure and Applied Math., Vol. 117, Queen's University, Kingston, Ontario, Canada, 2000.

[AJL2] — Duality and flat base change on formal schemes, Contemporary Math., Vol. 244, Amer. Math. Soc., Providence, R.I. (1999), 3-90.

[AJL3] Correction to the paper "Duality and flat base change on formal schemes", Proc. Amer. Math. Soc., Vol. 131, 351-357.

[AJL4] Greenlees-May duality on formal schemes, Contemporary Math., Vol. 244, Amer. Math. Soc., Providence, R.I. (1999), 93-112, 2002.

[C] B. Conrad, Grothendieck Duality and Base Change, Lecture Notes in Math., no. 1750, Springer, New York, 2000.

[D1] P. Deligne, Cohomology à support propre, et construction du foncteur $f^{!}$, appendix to R. Hartshorne's Residues and Duality, Lecture Notes in Math., no. 20, Springer-Verlag, Heidelberg, 1966.

[D2] Cohomology à supports propre, SGA4 Tome3, Lecture Notes in Math., no. 305, Springer-Verlag, New York, 1973.

[D3] - Deligne's notes on Nagata's Compactification. Notes by B. Conrad. Unpublished.

[GD] A. Grothendieck and J. Dieudonné, Élements de Géométrie Algébrique I, Springer-Verlag, New York, 1971.

[EGA-III] — Élements de Géométrie Algébrique III, Publications Math. IHES 11, Paris, 1961.

[EGA-IV] _ Élements de Géométrie Algébrique IV, Publications Math. IHES 20, Paris, 1964.

[LNS] J. Lipman, S. Nayak, P. Sastry, Pseudofunctorial behaviour of Cousin complexes on formal schemes. Available at http://www.math.purdue.edu/ ${ }^{\sim}$ ipman.

[Lu] W. Lütkebohmert, On compactification of schemes, Manuscripta Math., 80 (1993) 95111.

[M] H. Matsumura, Commutative ring theory, Cambridge Univ. press, Cambridge, 1986.

[N] M. Nagata, Imbedding an Abstract Variety in a Complete Variety, J. Math. Kyoto Univ., 2 (1962) $1-10$.

[Nay] S. Nayak, Pasting of pseudofunctors. In Preparation.

[Hrt] R. Hartshorne, Residues and Duality, Lecture Notes in Math., no. 20, Springer-Verlag, New York, 1966.

[Hu1] I-C. Huang, Pseudofunctors on modules with zero dimensional support, Memoirs, no. 548, Amer. Math. Soc., 1995

[Hu2] (2001), 310-354.

[S] P. Sastry, A pointwise criterion for dualizing pairs, Appendix to An explicit construction of the Grothendieck residue complex by A. Yekutieli, 117-126, Astérisque 208 (1992).

[Su] K. Suominen, Localization of sheaves and Cousin complexes, Acta mathematica, 131 (1973), 1-10.

[Lp1] J. Lipman, Dualizing sheaves, Differentials and Residues on Algebraic Varieties, Astérisque 117 (1984).

[V] J. L. Verdier, Base change for twisted inverse image of coherent sheaves, in Algebraic Geometry, Oxford Univ. press, 1969, pp. 393-408.

[Y] A. Yekutieli, Smooth formal embeddings and the residue complex, Canadian J. Math. 50 (1998), 863-896. 